\title{
A NEW APPROACH TO FABRICATE PDMS STRUCTURES USING FEMTOSECOND LASER
}

\author{
By \\ Hamsapriya Selvaraj \\ Bachelor of Engineering (Mechanical) \\ Bharathiyar University, India 2002 \\ Master of Engineering (Industrial) \\ Anna University, India 2005
}

\author{
A dissertation \\ presented to Ryerson University \\ in partial fulfillment of the requirement for the degree of \\ Doctor of Philosophy \\ in the program of \\ Aerospace Engineering
}

Toronto, Ontario, Canada, 2013

(C) Hamsapriya Selvaraj 2013 


\section{AUTHOR'S DECLARATION}

I hereby declare that I am the sole author of this dissertation. This is a true copy of the dissertation, including any required final revisions, as accepted by my examiners.

I authorize Ryerson University to lend this dissertation to other institutions or individuals for the purpose of scholarly research.

I further authorize Ryerson University to reproduce this dissertation by photocopying or by other means, in total or in part, at the request of other institutions or individuals for the purpose of scholarly research.

I understand that my dissertation may be made electronically available to the public.

\section{Hamsapriya Selvaraj}

Department of Aerospace Engineering

\section{Ryerson University}




\title{
ABSTRACT \\ A NEW APPROACH TO FABRICATE PDMS STRUCTURES USING FEMTOSECOND LASER
}

\author{
Doctor of Philosophy, 2013 \\ Hamsapriya Selvaraj \\ Department of Aerospace Engineering, Ryerson University
}

Polydimethylsiloxane (PDMS) is commonly used to prototype micro and nano featured components due to its beneficial properties. PDMS based devices have been used for diverse applications such as cell culturing, cell sorting and sensors. Motivated by such diverse applications possible through pure PDMS and reinforced PDMS, numerous efforts have been directed towards developing novel fabrication techniques. Prototyping 2D and 3D pure and reinforced PDMS microdevices normally require a long curing time and must go through multiple steps. This research explores the possibility of fabricating microscale and nanoscale structures directly from PDMS resin using femtosecond laser processing. This study offers an alternative fabrication route that potentially lead to a new way for prototyping of pure and reinforced PDMS devices, and the generation of hybrid nanomaterials.

In depth investigation of femtosecond laser irradiation of PDMS resin reveals that the process is highly intensity-dependent. At low to intermediate intensity regime, femtosecond laser beam is able to rapidly cure the resin and create micron-sized structures directly from PDMS resin. At higher intensity regime, a total break-down of the resin material occurs and leads to the formation of PDMS nanoparticles.

This work demonstrates a new way of rapid curing of PDMS resin on a microsecond timescale using femtosecond laser irradiation. The proposed technique permits maskless singlestep curing and is capable of fabricating 2D and 3D structures in micro-scale. Reinforced PDMS 
microstructures also have been fabricated through this method. The proposed technique permits both reinforcement and rapid curing and is ideal for fabricating reinforced structures in microscale. The strength of the nanofiber reinforced PDMS microstructures has been investigated by means of Nanoindentation test. The results showed significant improvement in strength of the material.

Hybrid PDMS-Si and hybrid PDMS-Al nanoparticle aggregate were generated using femtosecond laser. The results indicate that the hybrid PDMS nanostructures are clusters of nanoparticles that agglomerate and interweave three-dimensionally and also the possibility of formation of Si/Al nanoparticles enclosed in PDMS Shells. Presence of PDMS in the final hybrid structure is confirmed by micro-raman analysis. The versatility of our technique opens a new pathway to generate hybrid 3D fibrous nanostructures on any materials. 


\section{ACKNOWLEDGEMENT}

I wish to express my sincere appreciation to those who have contributed to this thesis and supported me in one way or the other during this amazing journey.

Foremost, I would like to express my sincere gratitude to my advisor Dr.Bo Tan for the continuous support of my Ph.D study and research, for her patience, motivation, enthusiasm, and immense knowledge. Her guidance helped me in all the time of research and writing of this thesis. I could not have imagined having a better advisor and mentor for my Ph.D study. Her advice on both research as well as on my career have been invaluable.

My sincere gratitude is reserved for Dr.Krishnan Venkatakrishnan for giving me the opportunity to carry out my doctoral research and for the financial support. I will never forget his support and for providing me numerous opportunities to learn and develop.

I would like to thank my examination committee: Dr.Muthukumaran Packirisamy (Concordia University), Dr.Huu Doan, Dr.Ziad Saghir, Dr.Puren Ouyang and Dr. Jeff Xi for their encouragement, insightful comments, and hard questions.

A special thanks to my family. Words cannot express how grateful I am to my parents and brother for all of the sacrifices that you've made on my behalf. Your prayer for me was what sustained me thus far.

I would like to acknowledge the most important person in my life - my husband Thirumurugan. Thank you for supporting me for everything, and especially I can't thank you enough for encouraging me throughout this experience. Thiru has been a constant source of strength and inspiration. There were times during the past five years when everything seemed hopeless and I didn't have any hope. I can honestly say that it was only his determination and constant encouragement that ultimately made it possible for me to see this project through to the 
end. Thiru has been a true and great supporter and has unconditionally loved me during my good and bad times. He has been non-judgmental of me and instrumental in instilling confidence. He has faith in me and my intellect even when I felt like digging hole and crawling into one because I didn't have faith in myself. These past several years have not been an easy ride, both academically and personally. I truly thank Thiru for sticking by my side, even when I was irritable and depressed. I feel that what we both learned a lot about life and strengthened our commitment and determination to each other and to live life to the fullest.

Special thanks to my beloved daughter Krishika and son Vihaan, who had to sacrifice their time and needs to support me in finishing my studies.

I would especially like to thank my brothers Senthilkumar, Jaganathan and my friend Murugesan for their unconditional support.

Last but not least I would like to give ultimate thanks to god. He guided and encouraged me. I thank him for his love and continuous support. Every breath I take and every moment I have are because of him. I would like to express the deepest thanksgiving to god and I pray he will continue to teach me and use me for his glory. Thank you, Lord. 


\section{LIST OF PUBLICATIONS}

$\checkmark$ Hamsapriya Selvaraj, Bo Tan and Krishnan Venkatakrishnan (2012) Self-Enclosed Nanofiber-Reinforced PDMS Channels Using Ultrafast Laser Irradiation, J. Micromechanical systems IEEE/ASME, Vol.21, Issue 5, pp: 1071 - 1076.

$\checkmark$ Hamsapriya Selvaraj1, Bo Tan and K Venkatakrishnan (2011) Maskless direct microstructuring of PDMS by femtosecond laser localized rapid curing, J. Micromech. Microeng, Vol.21, Issue 7.

$\checkmark$ Hamsapriya Selvaraj, Bo Tan and Kirshnan Venkatakrishnan(2011) Synthesis of nanofiber-filled polydimethylsiloxane using ultrafast laser irradiation, J Polym Res, Vol. 18, Issue 6, pp:1659-1665.

$\checkmark$ Hamsapriya Selvaraj, Bo Tan and Krishnan Venkatakrishnan (2012) Synthesis of hybrid PDMS-Si \& hybrid PDMS-Al fibrous nanostructured material using femtosecond laser ablation (Submitted to Polymer journal). 


\section{TABLE OF CONTENTS}

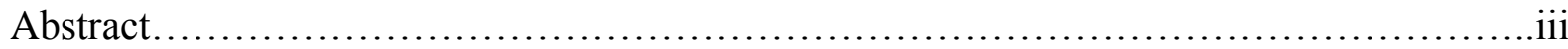

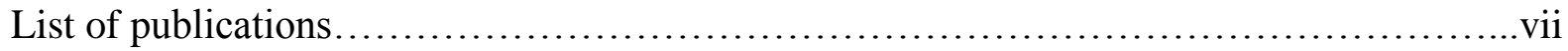

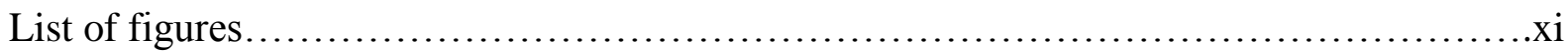

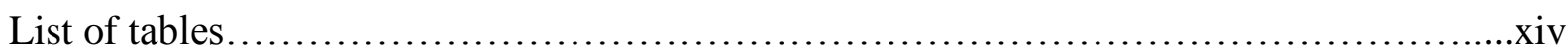

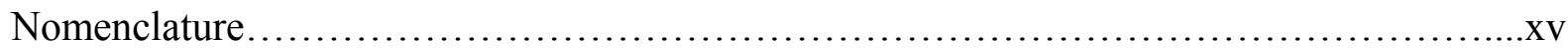

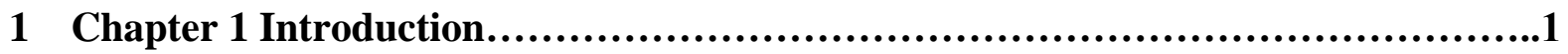

1.1. Biomedical micro-device materials and fabrication................................

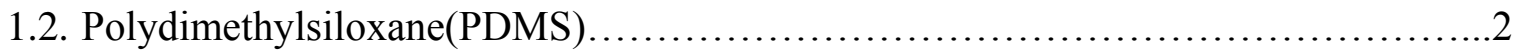

1.2.1. Molecular structure of PDMS .................................................

1.2.2. Physico-Chemical properties of PDMS ..................................

1.2.2.1. Intramolecular interactions ....................................

1.2.2.2. Intermolecular interactions................................... 4

1.2.3. Mechanical and thermal properties of PDMS ...............................5

1.3 Soft lithography for PDMS biomedical microdevice fabrication...................6

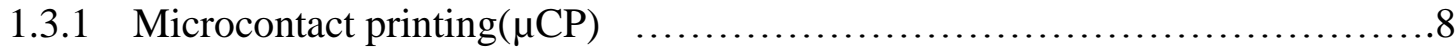

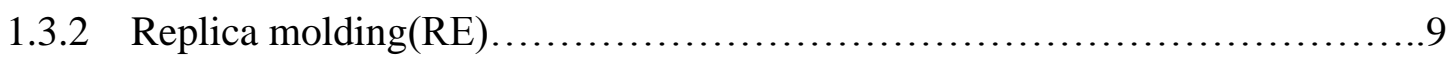

1.3.3 Solvent assisted micromolding(SAMIM) ..............................11

1.3.4 Fabrication of PDMS 3D microstructures.................................13

1.3.5 Summary of soft lithography of PDMS..................................16

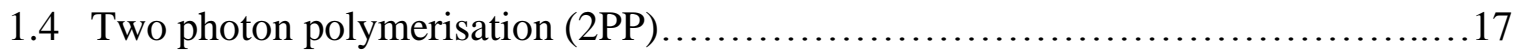

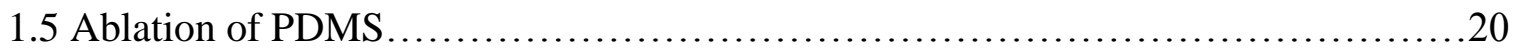

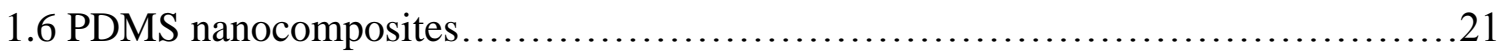

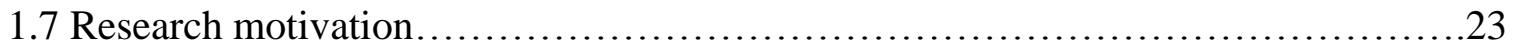


1.8 Research objective

2 Chapter 2 Thermal curing of PDMS..........................................25

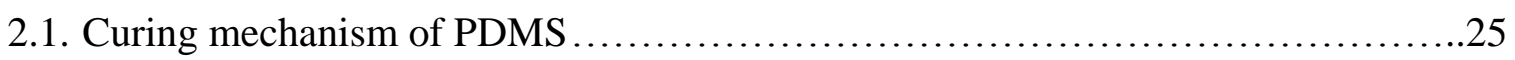

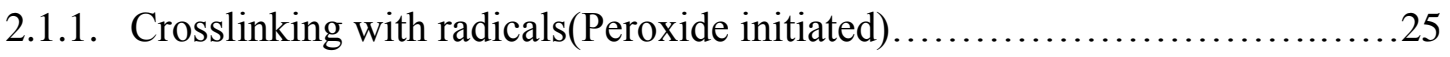

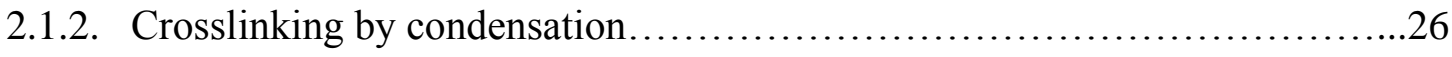

2.1.3. Crosslinking by addition......................................... 27

2.1.4. Crosslinking by irradiation......................................... 27

2.2. Characteristics of conventional curing techniques............................28

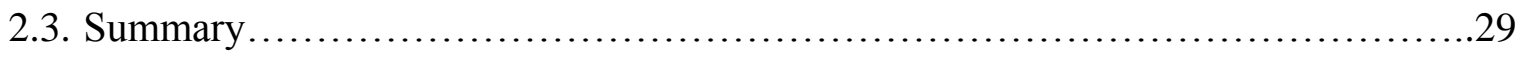

3 Chapter 3 Description of experimental setup....................................30

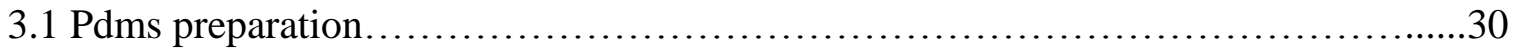

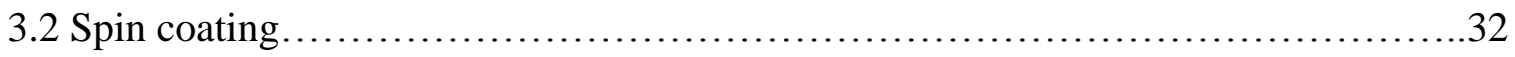

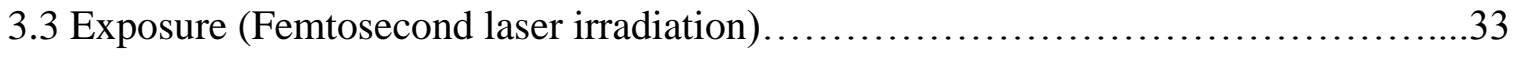

3.3.1. Setup 1- Low and intermediate intense irradiation of PDMS .................34

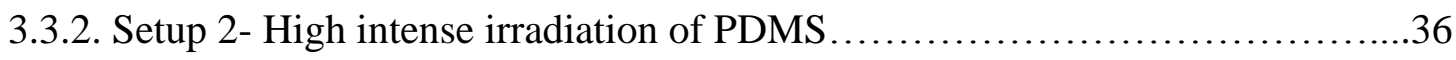

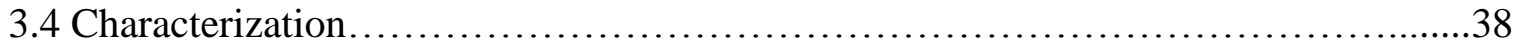

4 Chapter 4 Irradiation with low to intermediate intensity laser fluence.................39

4.1 Low intense irradiation: rapid curing of 2D and 3D microstructures $\ldots \ldots \ldots \ldots \ldots \ldots . . .39$

4.2 Heat transfer analysis of rapid heating at the volume in the vicinity of laser focus....40

4.3 Discussions: strength and degradation of PDMS $\ldots \ldots \ldots \ldots \ldots \ldots \ldots \ldots \ldots \ldots \ldots \ldots .48$

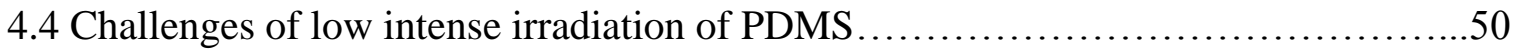

4.5 Intermediate intense irradiation of PDMS: rapid curing and laser ablation...........50

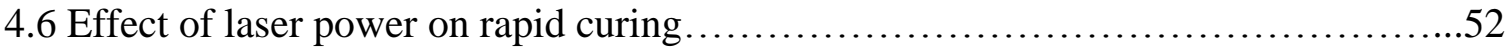




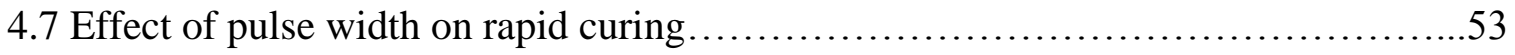

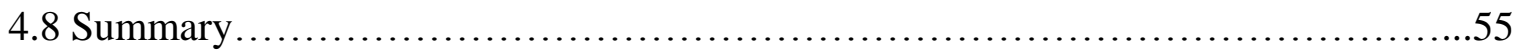

5 Chapter 5 Reinforcement of PDMS with nanofibers...........................57

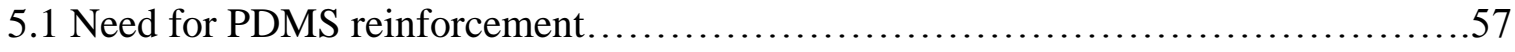

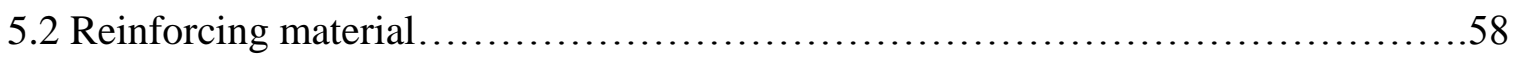

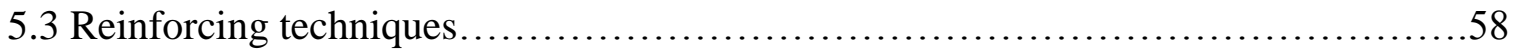

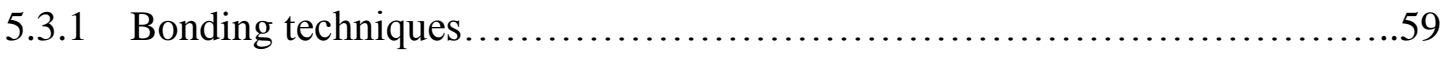

5.4 Proposed method: fabrication of reinforced PDMS microstructures...............62

5.4.1 Generation of nanostructures using laser ablation.......................62

5.4.2 Reinforcement of PDMS ..............................................

5.4.3 Reinforced structure fabrication: Self enclosed reinforced PDMS channels...65

5.4.4 Test for mechanical properties of reinforced polymer.....................71

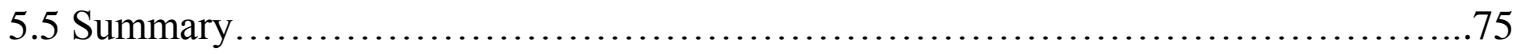

6 Chapter 6 Irradiation of high laser fluence: Synthesis of PDMS-Si/Al hybrid fibrous

nanostructure..................................................................

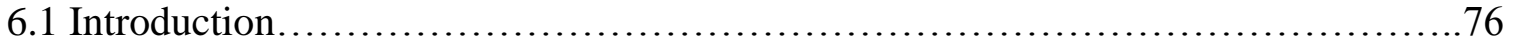

6.2 Hybrid nanostructure generation........................................ 78

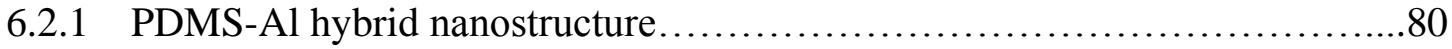

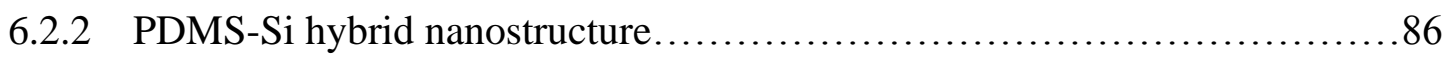

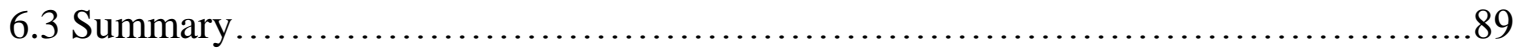

7 Chapter 7 Summary and future work.........................................91

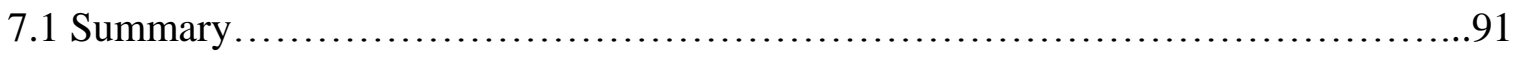

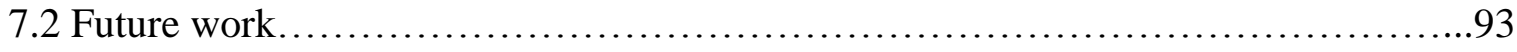

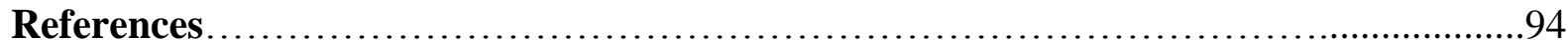




\section{LIST OF FIGURES}

Fig 1.1 Structure of PDMS with 3-D representation of silicone backbone (Orange: Si, Red:O,

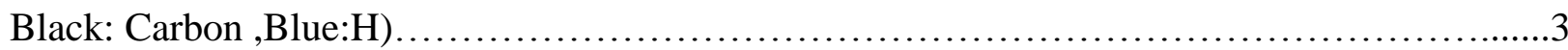

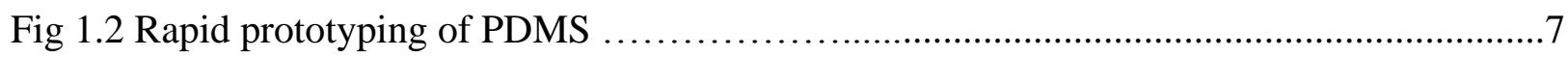

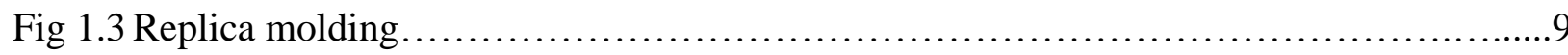

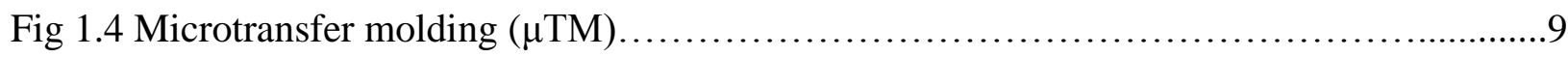

Fig 1.5 Examples of the structures that can be replicated using Microtransfer molding.............10

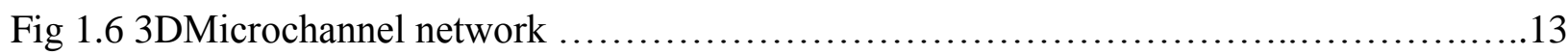

Fig.1.7 The membrane sandwich method for making a three-level channel system in a single membrane. (A) The top master contains one level of features and bottom master is a positive relief of photoresist containing two levels of features, and is made using two-level photolithography (B) Schematic diagram of the three-level channel system in a PDMS membrane. (C) Optical image (looking down the z-axis) of the PDMS membrane. (D) SEM of the channel system in polymer.

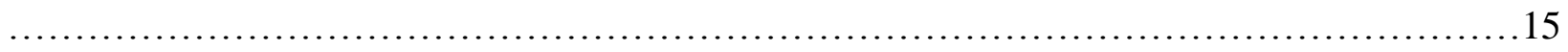

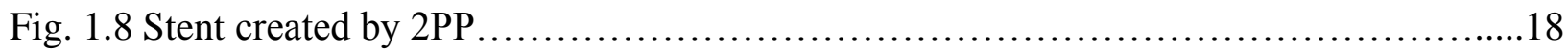

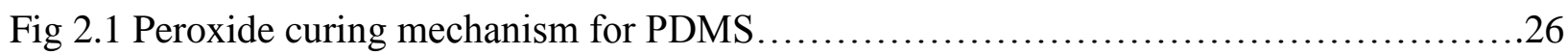

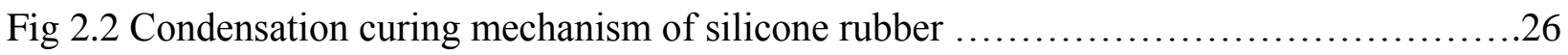

Fig 2.3 Addition curing mechanism of silicone rubber....................................27

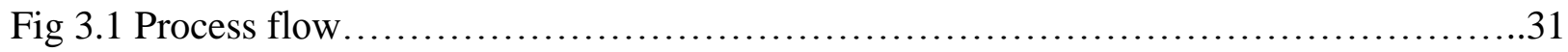

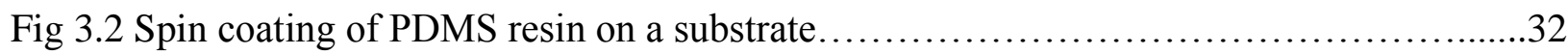

Fig3.3 Schematic representation of laser setup for low and intermediate irradiation of

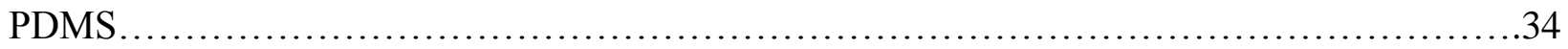


Fig 3.4 Laser spot size and depth of focus for converging laser beam using a telecentric lens. .36

Fig 3.5 Schematic representation of laser setup for High intense irradiation of PDMS. AOM, L, WP, M, D, GS, TL and TS denote acousto-optic modulator, lens, waveplate, mirror, diaphragm,

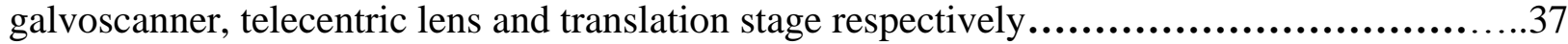

Fig 4.1 Position of the focused laser spot a) free standing structure b) surface structure........40

Fig 4.2 Surface Structure....................................................... 41

Fig 4.3 Freestanding structures.................................................... 42

Fig $4.4 \mathrm{Z}$ axis depth Vs Temperature at centre of Gaussian spot...........................45

Fig 4.5 a) Simulation Setup B) Simulated Model.......................................46

Fig 4.6.Temperature history of polymer resin after the laser irradiation......................47

Fig 4.7 Gaussian profile illustration....................................................

Fig 4.8 PDMS microchannel fabricated using proposed method $\ldots \ldots \ldots \ldots \ldots \ldots \ldots \ldots \ldots \ldots \ldots 1$

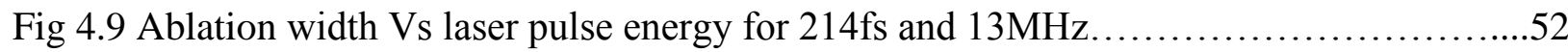

Fig 4.10 Rep rate: $13 \mathrm{MHz}$, scanning Speed: $1000 \mu \mathrm{m} / \mathrm{s}$, pulse width $214 \mathrm{fs}$ for different laser

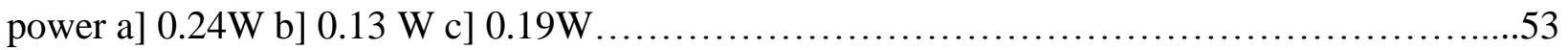

Fig 4.11 Rep rate: $13 \mathrm{MHz}$, scanning Speed: $1000 \mu \mathrm{m} / \mathrm{s}$, pulse width $214 \mathrm{fs}$ for different laser

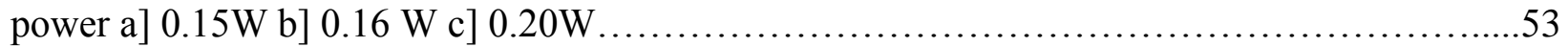

Fig 4.12 Rep rate: $13 \mathrm{Mhz}$, scanning Speed: $1000 \mu \mathrm{m} / \mathrm{s}$, pulse width $714 \mathrm{fs}$ for different laser

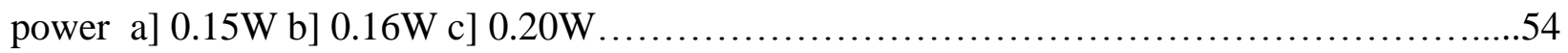

Fig 4.13 Rep rate: $13 \mathrm{MHz}$, scanning Speed: $1000 \mu \mathrm{m} / \mathrm{s}$, pulse width $1428 \mathrm{fs}$ for different laser

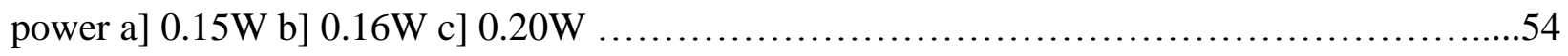

Fig 5.1 a)Generation of nanofibers using femtosecond laser ablation. .......................64

Fig 5.1 b)SEM image of a nanoparticles aggregate after laser ablation of silicon substrate......64

Fig 5.2 Reinforcement of PDMS with silicon nanofibers.............................65

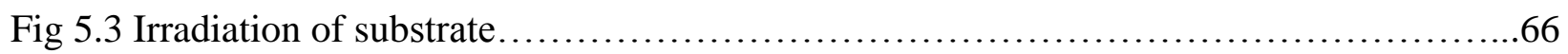

Fig 5.4 SEM Image of final microstructure created using this new process..................67 
Fig 5.5 Linking mechanism. .68

Fig 5.6 EDX analysis of a) reinforced PDMS microstructures (Spectrum 2) and b) pure PDMS microstructure (Spectrum 1).

Fig 5.7 SEM image of reinforced self enclosed channels a) 714 fs- $13 \mathrm{MHz}-200 \mathrm{~mW}$, b) $1428 \mathrm{fs}$ $13 \mathrm{MHz}-250 \mathrm{~mW} \& \mathrm{c}) 1428 \mathrm{fs}-13 \mathrm{MHz}-270 \mathrm{~mW}(\mathrm{ZOOM}$ Image $) \ldots \ldots \ldots \ldots \ldots \ldots \ldots \ldots \ldots . \ldots . \ldots \ldots$

Fig 5.8a) Load-displacement curve............................................... 72

Fig 5.8b) Curve for the applied force and penetration depth.............................

Fig 5.9 Optical microscopy image a] PDMS without fibers b] with fibers.....................74

Fig 6.1 Proposed single step direct synthesis of hybrid nanostructure.......................79

Fig 6.2 SEM micrographs of the laser irradiated PDMS coated aluminum sample surface and the nanoparticles aggregate respectively. (a) Surface featuring web like nanoparticles around the laser spot, (b, c) PDMS-Al /Al nanoparticles in the aggregate structure.....................83

Fig 6.3 TEM-EDX analysis of the nanoparticles in the aggregate structure (PDMS coated

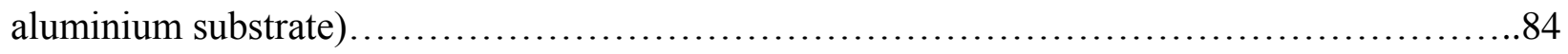

Fig 6.4 Raman Spectra for treated and untreated PDMS coated Al sample. ...................85

Fig 6.5 SEM and TEM micrographs of the laser irradiated PDMS coated silicon sample surface and the nanoparticles aggregate respectively. (a) Surface featuring web like nanoparticles around the laser spot, (b, c) PDM-Si /Si nanoparticles in the aggregate structure.

Fig 6.6 TEM-EDX analysis of the nanoparticles in the aggregate structure (PDMS coated Si substrate) .88 


\section{LIST OF TABLES}

Table 1.1.Comparison of PDMS permeability with other polymers.........................5

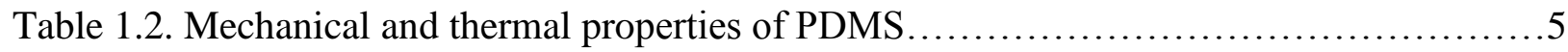

Table 2.1.Curing Parameters for PDMS prototyping (Conventional methods)................28

Table 4.1 Thermo physical properties............................................43 


\section{NOMENCLATURE}

A Projected contact area $\left(\mu \mathrm{m}^{2}\right)$

$\mathrm{A}_{\mathrm{s}} \quad$ Irradiated Area $\left(\mu \mathrm{m}^{2}\right)$

D Laser beam diameter(mm)

$\mathrm{D}_{\mathrm{o}} \quad$ Laser minimum spot diameter $(\mu \mathrm{m})$

E Young's modulus(GPa)

$\mathrm{E}_{\mathrm{r}} \quad$ Reduced modulus $(\mathrm{Pa})$

f $\quad$ Effective focal length (mm)

fs $\quad$ Femtosecond

h $\quad$ Film thickness( $\mu$ icrons)

$\mathrm{h}_{\max } \quad$ Maximum depth of indentation

$\mathrm{h}_{\mathrm{t}} \quad$ Heat transfer coefficient $\left(\mathrm{W} / \mathrm{m}^{2} \mathrm{~K}\right)$

$\mathrm{I}_{\mathrm{O}} \quad$ Incident Laser radiation intensity $\left(\mathrm{W} / \mathrm{cm}^{2}\right)$

$\mathrm{k} \quad$ Thermal diffusivity of PDMS $\left(\mathrm{m}^{2} \mathrm{~s}^{-1}\right)$

L Diffusion length $(\mu \mathrm{m})$

$\mathrm{mW} \quad$ milliwatt

$\mu \mathrm{m} \quad$ micrometer

$\mu \mathrm{J} \quad$ micro joules

$\lambda_{\mathrm{o}} \quad$ Laser wavelength(nm)

$\mathrm{P}_{\max } \quad$ Peak indentation load 
S Unloading stiffness

$\mathrm{T}_{\mathrm{i}} \quad$ Curing threshold temperature(K)

T Initial average temperature $(\mathrm{K})$

$\mathrm{T}_{\infty} \quad$ Room temperature $(\mathrm{K})$

W Watts

$\mathrm{z} \quad$ Absorbed length along laser direction(Z-axis $)(\mu \mathrm{m})$

$\beta \quad$ Correction factor for Berkovich indenter

$\varepsilon \quad$ Material extinction coefficient

$\rho \quad$ Density of the polymer $\left(\mathrm{kg} / \mathrm{m}^{3}\right)$

$\mathrm{MHz} \quad$ Megahertz

$\tau_{\mathrm{p}} \quad$ Pulsewidth(fs) 


\section{CHAPTER 1}

\section{INTRODUCTION}

\section{1 Biomedical micro-device materials and fabrication}

Micro devices, often referred to as micro electro mechanical systems (MEMS), have traditionally been fabricated from silicon-based materials using processing techniques adapted from the semiconductor industry. It was initially developed for the production of sensors and actuators used in aircrafts and automobiles, as well as for environmental control. Its popularity exploded in the last two decades and its applications have branched out. One such an example is that Bio-MEMS are perceived as the most promising solution for high-quality healthcare at an acceptable cost [1]. Bio-MEMS and Bio-NEMS have undergone considerable growth since 1990s.

The new application of MEMS in biomedical field demands the adoption of a different category of materials in order to meet the requirement associated with such applications. Ideal materials for biomedical microdevices must be biocompatible, economical, easy to fabricate, soft and compliable, chemically modifiable, and multi-functional. Metals, ceramics, glasses and polymers have been utilized to develop biomedical microdevices.

Polymer is one of the most commonly used materials of biomedical microdevices, owning to its mechanical, chemical and other properties. A considerable number of polymers have been investigated for the fabrication of Bio-MEMS. Commercially developed PDMS based medical microdevice has been in the market for quite a while.

The most commonly used polymeric materials include polyamide(PA),

polybutylenetherepthalate(PBT), polycarbonate $(\mathrm{PC}), \quad$ polyethylene $\quad(\mathrm{PE})$, 
polyoxymethylene(POM), polypropylene (PP), polyphenylene ether (PPE), polystyrene (PS), polysulfone (PSU), polyetheretherketone (PEEK), and polyetherimide (PEI), Polydimethylsiloxane (PDMS), poly (methyl methacrylate) (PMMA), polyethylene terephthalate (PET), polycarbonate, acrylic plastics parylene, SU-8 etc. A newer material, cycloolefin copolymer (COC), also seems very promising for microdevices given its chemical stability and optical transparency [2-3]. Because of their diverse properties, different fabrication methods are used for different polymeric materials.

The conventional micro-manufacturing techniques that were developed for semiconductors are not suitable for biomedical microdevice fabrication because of the adoption of unconventional materials. Therefore, fabrication techniques that specifically fit to the biomedical microdevices have been developed. Biomedical devices are cost-sensitive and they are usually produced in small volumes, especially true for clinical applications. This posts difficult challenges for fabricator who has to produce low cost microdevices in small quantities. Therefore, rapid prototyping becomes critical for the fabrication of biomedical microdevices.

In the following sections, one of the most popular polymers used for biomedical microdevice, Polydimethylsiloxane (PDMS) will be introduced and the fabrication techniques will be reviewed.

\subsection{Polydimethylsiloxane - PDMS}

PDMS is optically transparent, durable, non-fluorescent, biocompatible, chemically inert and nontoxic. Besides its well-known superior elasticity and flexibility in mechanical applications, PDMS has become a popular choice for biological studies because of its nontoxicity to cells and high permeability to gases [4-5]. It also has been traditionally used as a biomaterial in catheters, drainage tubing, and insulation for pacemakers, membrane oxygenators, 
and ear and nose implants [6]. This elastomeric polymer has enabled the fabrication of micro devices with two dimensional as well as complicated three dimensional structures, such as channels, valves, pumps, mixers, and switches [7-13].

\subsubsection{Molecular structure of PDMS}

Polydimethylsiloxane (PDMS) belongs to the family of siloxane polymers. The discovery of siloxanes opened a fascinating field of materials at the interface of organic/inorganic chemistry [14]. Silicones have a Si-O-Si backbone to which, different organic groups can be attached through Si-C linkage giving silicones the general formula [-OSiR'R- $]_{\mathrm{n}}$ ( where $\mathrm{R}$ and $\mathrm{R}$ ' refers to the organic side groups and $\mathrm{n}$ is an integer denoting the number of repeating units). The presence of both the organic and inorganic components in a single molecule gives silicone unique properties [14].The $\mathrm{Si}-\mathrm{O}$ bond renders silicones to be highly flexible material because of its long bond length and a long bond angle that allows for ease changes in conformation. In addition, silicones are non- toxic, chemically inert and gas permeable. The most widely used silicone polymer is PDMS which has two methyl groups attached to each silicon atom as shown in Fig 1.1. PDMS is manufactured by reaction between silicon and methyl chloride at temperatures of $250-350^{\circ} \mathrm{C}$ and pressures of $1-5$ bars [14]. This reaction yields a mixture of methyl chlorosilanes. For obtaining PDMS, dimethyldichlorosilane is hydrolyzed, followed by polymerization of the oligomers obtained on hydrolysis.
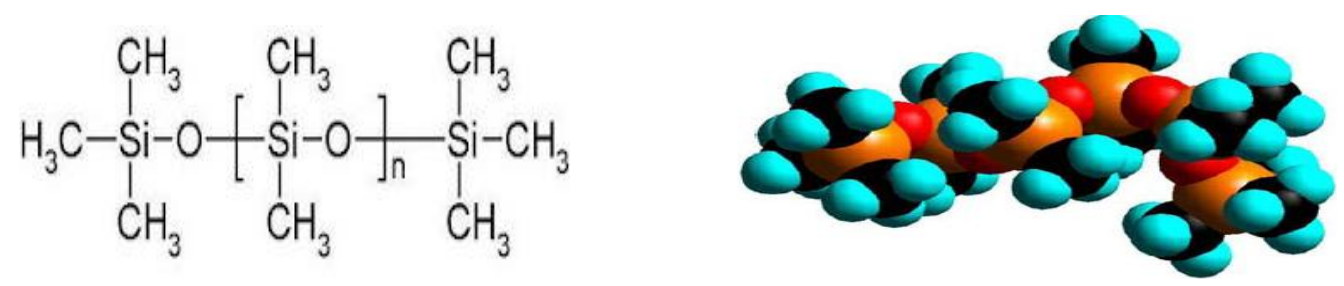

Fig 1.1 Structure of PDMS with 3-D representation of silicone backbone (Orange: Si, , Red:O, Black: Carbon ,Blue:H) [15] 


\subsubsection{Physico-Chemical Properties of PDMS}

\subsubsection{Intramolecular interactions}

The chemical formula for PDMS is $\mathrm{CH}_{3}\left[\mathrm{Si}\left(\mathrm{CH}_{3}\right)_{2} \mathrm{O}\right]_{n} \mathrm{Si}\left(\mathrm{CH}_{3}\right)_{3}$, where $n$ is the number of repeating monomer $\left[\mathrm{SiO}\left(\mathrm{CH}_{3}\right)_{2}\right]$ units. PDMS polymers are characterized by strong chemical bonds, not easily broken by homolytic scission because of their polarity. Only strong acids or strong bases are capable to depolymerise the siloxane chain. As a result, the PDMS polymers are not very susceptible to oxidation or thermal degradation and they can be sterilized by heat. While the siloxane backbone is made of very polar $\mathrm{Si}-\mathrm{O}$ bonds, the PDMS polymers are actually very hydrophobic as the methyl groups shield the polar backbone, a feature enhanced by the very low energy of rotation around a $\mathrm{Me}_{2} \mathrm{Si}-\mathrm{O}$ bond (rotation barrier $=3.3 \mathrm{~kJ} / \mathrm{mol}$ ) which allow PDMS polymers to reduce their surface energy and their surface tension by exposing a maximum number of methyl groups. The PDMS low surface tension property is used to prepare antifoam agents.

\subsubsection{Intermolecular interactions}

Because of its low rigidity, the siloxane backbone allows the methyl groups to be easily exposed to the outside and as a result, the PDMS polymers are characterized by low intermolecular interactions. This is demonstrated in several ways:

- Even at high molecular weight, the PDMS polymers are liquid;

- The PDMS polymers properties are not very temperature dependent;

- It is possible to alter the surface chemistry by additives, which is useful for creating reversible or irreversible bonds with other smooth surfaces.

The PDMS polymers, compared to other polymers, are very permeable to the diffusion of various substances, gases or active drugs. For example, without surface chemistry the intrinsic 
surface property of PDMS exhibits hydrophobic surface characteristics [16]. Table 1.1 compares the permeability of PDMS to other polymers that are used for biomedical applications.

Table 1.1 Comparison of PDMS permeability with other polymers [17-18]

\begin{tabular}{cccc}
\hline Type & $\begin{array}{c}\text { Permeability } \\
\text { to } \mathrm{O}_{2}\end{array}$ & $\begin{array}{c}\text { Permeability } \\
\text { to } \mathrm{CO}_{2}\end{array}$ & $\begin{array}{c}\text { Relative } \\
\text { permeability to } \\
\text { Progesterone }\end{array}$ \\
\hline Polydimethylsiloxane(PDMS) & 79 & 405 & 100 \\
Polyethylene & 0.002 & 0.007 & 0.1 \\
Polytetrafluoroethylene & 0.001 & 0.003 & 0.1 \\
\hline
\end{tabular}

\subsubsection{Mechanical and thermal properties of PDMS}

PDMS is soft, suitable for in vivo biomedical applications. For instance, its shear elastic modulus $\mathrm{G}$ is around $250 \mathrm{kPa}$ due to its glass transition temperature $\left(\mathrm{T}_{\mathrm{g}} \sim 125^{\circ} \mathrm{C}\right)$, the lowest among polymeric materials. The mechanical and thermal properties of PDMS are listed in Table 1.2.

Table 1.2 Mechanical and thermal properties of PDMS

\begin{tabular}{|c|c|c|}
\hline Property & Value & Reference \\
\hline Mass Density & $0.97 \mathrm{~kg} / \mathrm{m}^{3}$ & $\begin{array}{l}\text { Polymer Data Handbook, Mark } \\
\text { J.,Oxford Univ. Press,NewYork (1999)[19] }\end{array}$ \\
\hline Young's Modulus & $360-870 \mathrm{kPa}$ & $\begin{array}{l}\text { Re-configurable Fluid Circuits by } \\
\text { PDMS Elastomer Micromachining[20] }\end{array}$ \\
\hline Poisson Ratio & 0.5 & Polymer Data Handbook [19] \\
\hline Dynamic Viscosity & 3900 centipoise & http://www.dowcorning.com/ [21] \\
\hline Tensile /Fracture Strength & $2.24 \mathrm{MPa}$ & Polymer Data Handbook[19] \\
\hline Specific Heat & $1.46 \mathrm{~kJ} / \mathrm{kg} \mathrm{K}$ & Polymer Data Handbook[19] \\
\hline Thermal Conductivity & $0.15 \mathrm{~W} / \mathrm{mK}$ & Polymer Data Handbook[19] \\
\hline Adhesion to silicon dioxide & Excellent & $\begin{array}{l}\text { Re-configurable Fluid Circuits } \\
\text { PDMS Elastomer Micromachining[20] }\end{array}$ \\
\hline Melting Point & $-49.9-40^{\circ}$ & Knovel Critical Tables[22]. \\
\hline
\end{tabular}




\subsection{Soft Lithography for PDMS biomedical microdevice fabrication}

In the last decade, development of PDMS bio-medical devices mushroomed. Most PDMS-based micro devices are fabricated with soft lithography. Whitesides et al. [8] developed the original soft lithography techniques and subsequently used them to create micropatterns and microstructures from a variety of materials. Soft lithography represents a non photolithographic approach to micro and nano fabrication which is based on self assembly as well as replica molding. It normally requires a stamp, on which the patterns or structures is created. The stamp is then used as a mold to transfer the pattern onto an elastomeric material. Complex patterns or architectures can be transferred. Soft lithography has been demonstrated to be capable of obtaining features between $30-80 \mathrm{~nm}$. It is extremely convenient for the rapid replication of micro devices with features less than $10 \mu \mathrm{m}$. Soft lithography provides a convenient, effective, and low-cost method for microstructure fabrication. It has many variations and capable of mass production of two-dimensional planary microstructures. To build three-dimensional devices, planary microstructures made from soft lithography are stacked layer-by-layer.

Soft lithography requires multiple steps from the development of appropriate masters to a working device. Figure 1.2 illustrated the fabrication process of PDMS devices. A photoresist master is first fabricated using photolithography or another micro/nanofabrication procedure. The PDMS prepolymer mixed with a curing agent was poured over the master and allowed to cure. The master pattern is transfered to the PDMS creating a negative replica of the original pattern. 


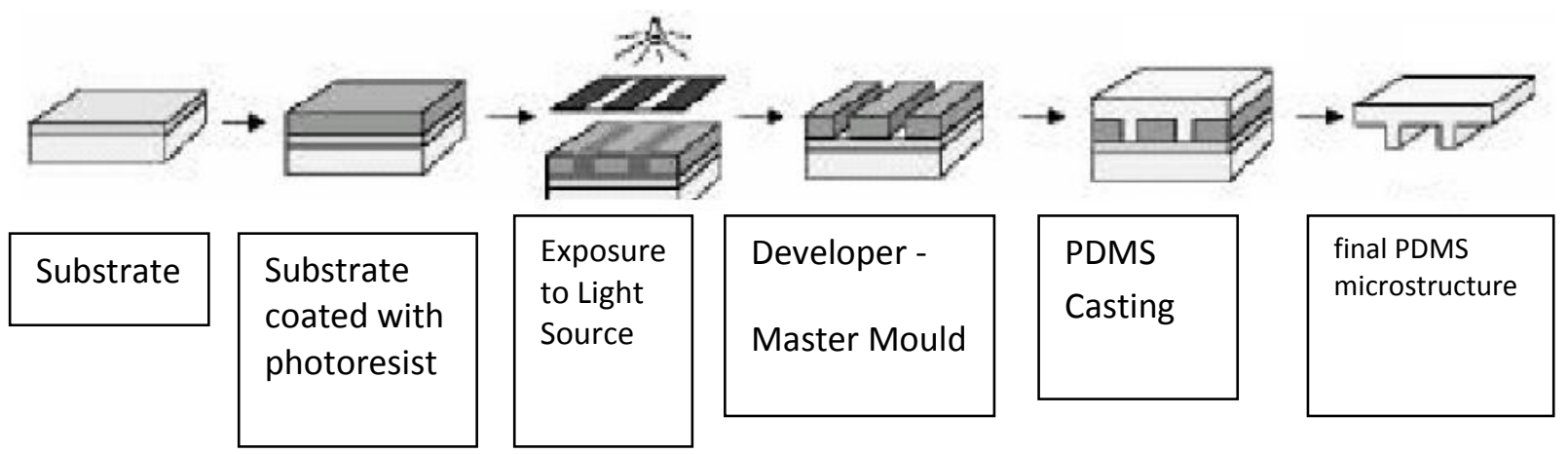

Fig 1.2 Rapid prototyping of PDMS [8]

The process begins by designing a pattern via computer aided design software, which is then printed on high resolution transparency. This transparency is used as photomask in standard lithography. In photolithography a thin photosensitive polymer layer [Photoresist] is illuminated with UV light through the photomask to initiate a polymerization process in a pattern at defined regions. The unpolymerized photoresist is then dissolved in a developer solution. This leaves relief structure on a silicon wafer. By using different photoresists [24], features could be generated with thickness between 1 and $20 \mu \mathrm{m}$. Alternatively solid printing can be used to create the master. Using transparencies printed from a high resolution printer, it could rapidly and reliably create features with widths greater than $20 \mu \mathrm{m}$. The relief structure on the substrate is then used as a master to cast PDMS. This involves casting of the mixed, degassed PDMS prepolymer on the master, heat curing and eventually peeling the casted PDMS off the master.

Several different techniques are collectively described as soft lithography: Near-Field Phase Shift Lithography, Replica Molding (REM), Micromolding in Capillaries (MMIC), Microtransfer Molding $(\mu \mathrm{TM})$, Solvent-assisted Microcontact Molding (SAMIM), and Microcontact Printing ( $\mu \mathrm{CP})$. They are as described below. 


\subsubsection{Micro Contact printing $(\mu \mathrm{CP})$}

Microcontact printing (or $\mu \mathrm{CP}$ ) is a form of soft lithography that uses the relief patterns on a master Polydimethylsiloxane (PDMS) stamp to form patterns of self-assembled monolayer's (SAMs) of ink on the surface of a substrate through conformal contact. Its applications are wide ranging including microelectronics, surface chemistry and cell biology. In micro contact printing $(\mu \mathrm{CP})$, first PDMS master is prepared using standard lithographic technique. Then inking of stamp occurs through the application of a thiol solution either by immersion or coating the stamp with a Q-tip. The highly hydrophobic PDMS material allows the ink to be diffused into the bulk of the stamp, which means the thiols reside not only on the surface, but also in the bulk of the stamp material. This diffusion into the bulk creates an ink reservoir for multiple prints. The stamp is let dry until no liquid is visible and an ink reservoir is created and then brought in contact with a substrate. The patterned thiol ink forms a selfassembled monolayer (SAMs) on the surface of the substrate, which can be used as passivating layer for selective material growth or deposition or as thin masks for selective etching. Since the PDMS stamp is "soft", the substrate surface can be either planar or curved. Microcontact Printing has several advantages including: the simplicity and ease of creating patterns with micro-scale features, can be done in a traditional laboratory without the constant use of a cleanroom (cleanroom is needed only to create the master), multiple stamps can be created from a single master, individual stamps can be used several times with minimal degradation of performance, a cheaper technique for fabrication that uses less energy than conventional techniques. It also has some disadvantages including: stamp deformation, substrate contamination, shrinking /swelling of the stamp and ink mobility [25-26, 11]. 


\subsubsection{Replica Molding (REM)}

Replica molding (Fig 1.3) can be defined as the process that transfers a pattern from a rigid or elastomeric mold in to another material by way of a liquid solidifying when in contact with the mold. It is a procedure that accommodates a wider range of materials than does photolithography. It also allows duplication of three-dimensional topologies in a single step, whereas photolithography is not able to replicate such structures [8]. It has been used for the mass production of surface relief structures such as diffraction gratings, holograms, compact disks (CDs) and microtools [27-29]. The fidelity of replica molding is determined by van der Waals interactions, wetting, and kinetic factors such as filling of the mold. These physical interactions should allow more accurate replication of features that are smaller than $100 \mathrm{~nm}$ than does photolithography, which is limited by optical diffraction. It can be further broken down in to subfields microtransfer molding and micro molding in capillaries.

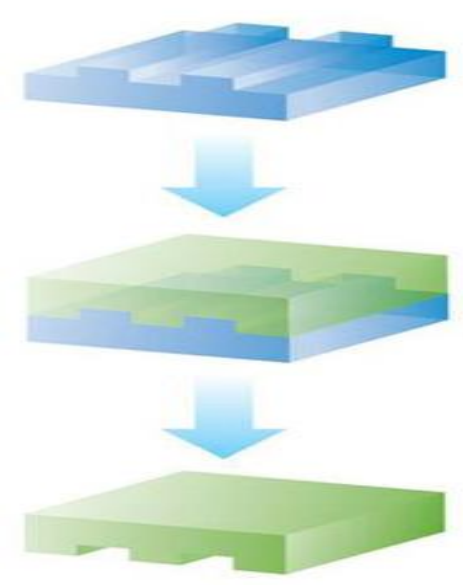

Fig 1.3 Replica Molding (REM) [8]

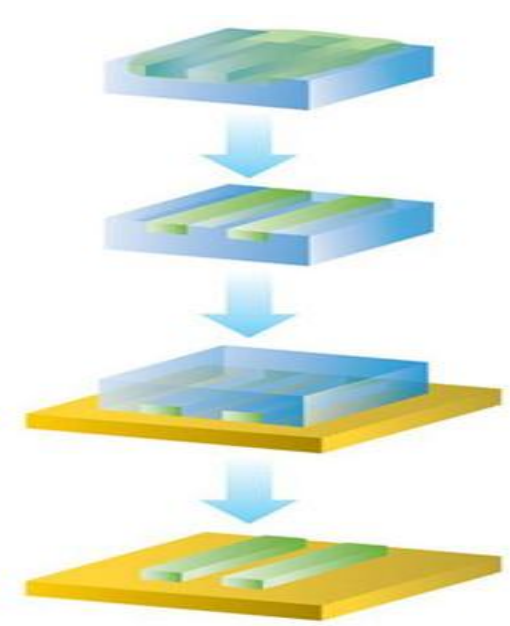

Fig 1.4 Microtransfer molding ( $\mu \mathrm{TM})$ [8] 


\section{a) Microtransfer Molding ( $\mu \mathrm{TM})$}

Microtransfer Molding ( $\mu \mathrm{TM}$ ) (Fig 1.4) uses Polydimethylsiloxane (PDMS) mold as a master and the recessed regions of an elastomeric mold are first filled with a prepolymer or ceramic precursor. An example of the liquid prepolymer is polyurethane, or any thermally curable epoxy. The excess polymer is removed from the master and the mold is then placed on a substrate. The filling material is then cured and the mold is removed. The residual material on the substrate surface forms relief patterns replicating the mold. Like $\mu \mathrm{CP}, \mu \mathrm{TM}$ can fast produce small structures on a large scale. It can also be used to build 3D structures layer by layer. One of the best examples of this is done by John Fourkas' group at University of Maryland, which produced the structures as shown in the Fig 1.5 [30]. The structures have significant overhang and are hollow. This is made possible by incorporating a thin membrane into the master, so that the mold is able to separate from the replica after curing. The mold must also be flexible enough to deform so that it can release from overhangs [30].

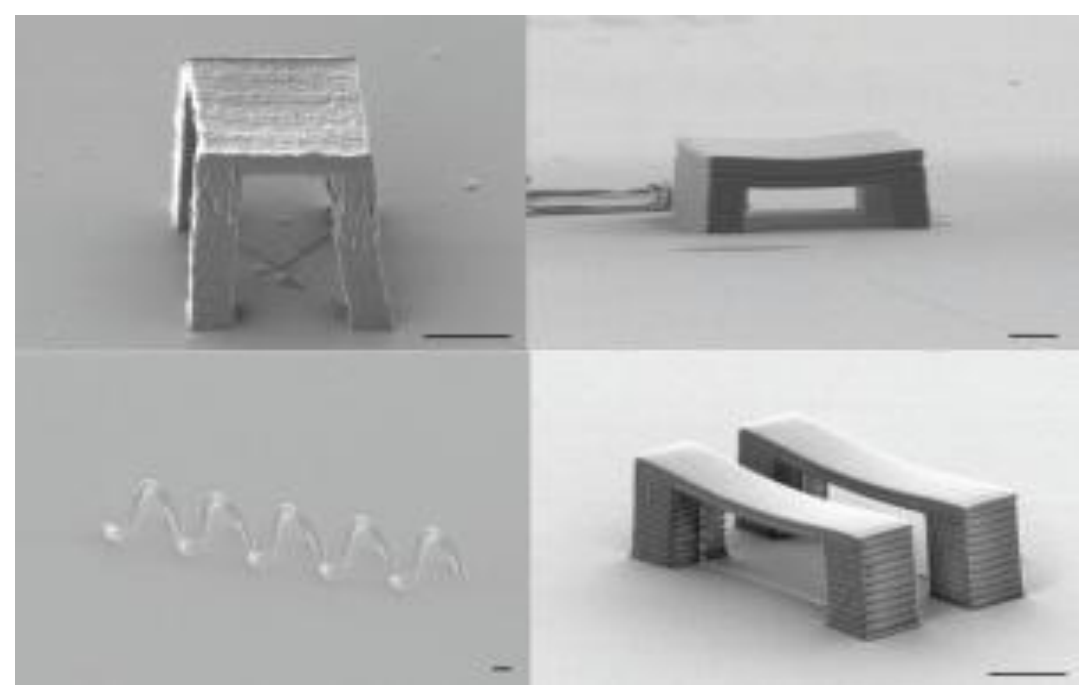

Fig 1.5 Examples of the structures that can be replicated using Microtransfer molding [30]. 


\section{b) Micro molding in capillaries (MMIC)}

Micromolding in Capillaries, also known as MMIC, is a soft lithographic technique which utilizes the thermodynamically driven process of capillary filling. A liquid (usually a prepolymer) will fill a capillary in order to minimize the solid-vapor and solid-liquid interfacial free energies. The rate of this capillary flow is dependent on the viscosity of the liquid, and both the length and diameter of the capillary. MMIC is similar to $\mu \mathrm{TM}$, however in this case the elastomeric master has a microchannel structure. Mould is brought in contact with a substrate. Curable polymers are introduced into channels formed by the mould and substrate through capillary action. The polymer is then cured and the mold in removed. By selectively introducing different material into different channels, arrays of different materials can be closed spaced through this method. It can also be used to selectively modify the surface of a substrate under the channels through introducing different solutions to the channels. Because of the nature of capillary filling, this technique does have some drawbacks: MMIC requires a connected network of capillaries; therefore, isolated structures cannot be formed, viscous drag of the liquid limits the distance in which capillary filling can be used; therefore, relatively long patterns are difficult to create with MMIC, the rate of filling is proportional to the diameter of the capillary; therefore, small capillaries result in very slow filling [8].

\subsubsection{Solvent-assisted micro molding (SAMIM)}

Solvent-assisted micromolding (SAMIM), like most of the previous techniques discussed, "allows fabrication of patterned, quasi-three-dimensional microstructures on the surfaces of polymeric substrates [8]." First, a substrate is evenly coated with a thin layer of polymer. An elastomeric PDMS mold is then wetted with a solvent that is both a good solvent for the polymer 
and a solvent that will not swell and thus distort the features on the PDMS mold. The mold is brought into contact with the surface of the polymer, the solvent dissolves a thin layer of the polymer surface and the polymer-solvent solution absorbs and dries onto the surface of the mold. While the mold is in direct contact with the polymer surface, the polymer solidifies to the surface of the mold as the solvent evaporates. Finally, the mold is removed to reveal a patterned polymer surface complementary to the mold. The overall effectiveness of SAMIM is the choice of the solvent. The solvent "should rapidly dissolve or swell the surface of the polymer; it should not, however, swell the PDMS mold and thereby distort the mold and/or destroy the conformal contact between the polymer and the mold." The solvent should also have a high vapor pressure and a moderately high surface tension, such as acetone, ethanol and methanol, to ensure quick dissipation of excess solvent and to minimize the swelling of the PDMS mold [8]." Whitesides et al [8] mention four main differences between SAMIM and existing techniques: SAMIM uses an elastomeric mold rather than a rigid mold, uses a solvent to "soften" the surface of the polymer and does not require specialized equipment or a system for pressing the mold into the substrate, does not suffer from slow rates of capillary filling, which limits MIMIC to relatively small areas, can be used with a wide range of polymers or prepolymers: the only requirement seems to be a solvent that dissolves the polymer of the substrate and wets the surface of the PDMS mold but does not significantly swell the mold. 


\subsubsection{Fabrication of PDMS 3D microstructures}

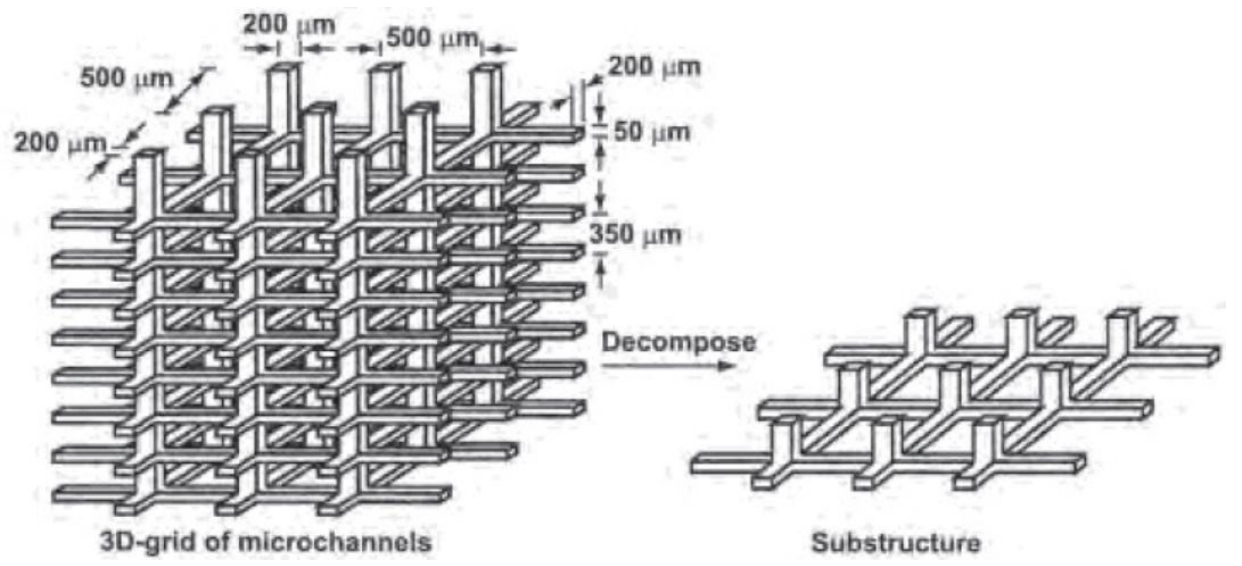

Fig. 1.6 3D microchannel network [31]

While soft lithography processes have found broad applications, they are generally limited to fabrication of two-dimensional (2D) or extruded 2D structures, including microfluidic channels and membrane-based micro actuators. These 2D structures must be built up and adhere to a supporting substrate. Since soft lithography is a surface relief manufacturing method, it is difficult to fabricate complicated three-dimensional structures or suspended mechanical structures. True three dimensional free standing structures cannot be achieved with soft lithography. However, many efforts have been exerted to fabrication of three dimensional complex microfluidic channel networks, where horizontal channels lay in different high within a thick substrate and vertical channels interconnects those horizontal channels. Fig. 1.6 gives an example of such a channel network. Fabrication of this kind of 3D channels cannot be done directly by soft lithography. The channel network will be decomposed into multiple layers. Each contains one set of horizontal channels and a segment of the vertical channels that used to connect those horizontal channels. Soft lithography is used to create each layer individually. Those individual layers are then stacked up and bond to each other. Many efforts have been 
attempted to build three-dimensional micro devices channels including: two level photolithography and the "membrane-sandwich" method.

When thinking about making topographic surface features within microfluidic channels, two-level photolithography comes to mind. Two-level photolithography fabricating process includes mask alignment and polymerization of SU-8 photoresist. This method makes grooved structures inside channels for chaotic mixing [32].

For PDMS chip, most 3D structure was fabricated by an add-on process, which stacks 2D patterned PDMS thin layers together. The "membrane sandwich" method was brought forward to suggest the structure of the final system by the help of two-level photolithography; developed for fabricating topographically complex 3-D channel structures in PDMS that stacks many thin patterned PDMS layers( Fig1.7) [33]. The "membrane sandwich" consisting of three levels of features within a single thin layer of PDMS (approximately $100 \mu \mathrm{m}$ ) is place between two thicker pieces of PDMS resulting in a very strong structural support. In this process two masters are fabricated with the use of the membrane. The top master is made by replica molding and silanized that have a single level of the features. The bottom master is made of silicon and photoresist by two-level photolithography containing two-levels of features. Fabrication of the membrane is accomplished by placing PDMS prepolymer between the two masters and applying a small amount of pressure. The pressure allows the masters to be in contact while at the same time the prepolymer is being expelled from the area of the initial contact. Possessing complimentary microscopic alignment tracks allows both masters to be slipped into one another without any form of magnification needed to accomplish this task. Following the curing process of the prepolymer the resulting silicon master is easily removed from the PDMS allowing the 
membrane to remain attached to the silanized PDMS master. Exposure of the membrane and a PDMS flat are then oxidized by plasma-treatment and brought into contact with one another. Once this step is completed the PDMS master is peeled away from the opposite side of the membrane and the membrane is sealed against another PDMS flat [32-33].
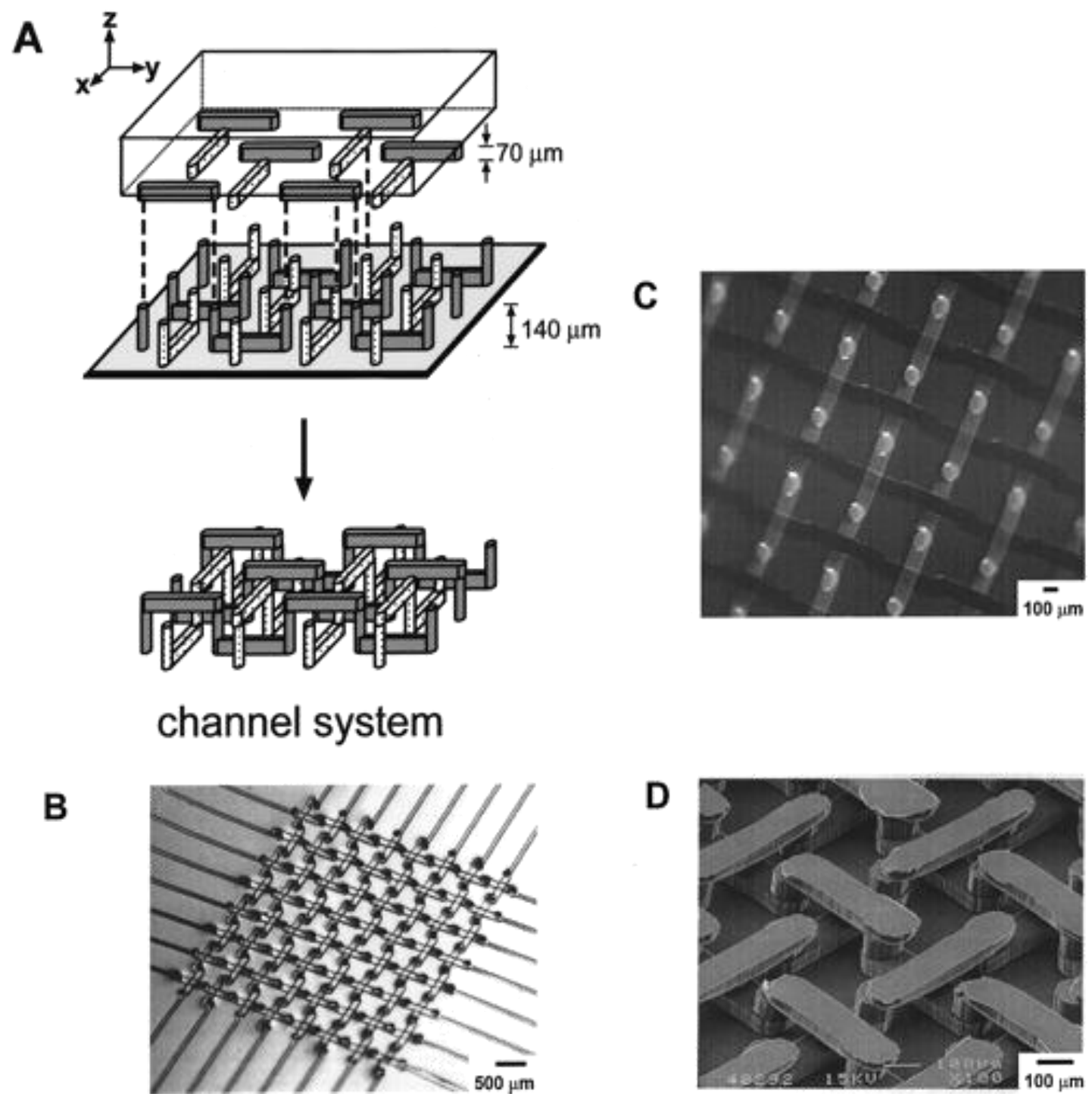

Fig.1.7 The membrane sandwich method for making a three-level channel system in a single membrane. (A) The top master contains one level of features and bottom master is a positive relief of photoresist containing two levels of features, and is made using two-level photolithography. (B) Schematic diagram of the three-level channel system in a PDMS membrane. (C) Optical image (looking down the z-axis) of the PDMS membrane (D) SEM of the channel system in epoxy polymer [32]. 


\subsubsection{Summary of soft lithography of PDMS}

Soft lithography is a versatile microfabrication technique, having many variations. It can be implemented to a wide variety of different polymers, not just PDMS. In addition to processing traditional polymers, significant process has also been made in micro patterning functional materials such as conducting polymers, piezoelectric polymers, biodegradable polymers, and hydrogels. Furthermore,

Some fundamental advantages of PDMS stamp method are that

- it is an inexpensive method for mass production;

- it can be applied to pattern large areas, greater than $20 \mathrm{~mm} \times 20 \mathrm{~mm}$;

- there is no diffraction limit to spatial resolution; features as small as 30nm have been fabricated;

- Generation and replication of three dimensional topologies are possible through layer-bylayer stacking of plenary microstructures;

- And it is capable to generate complex patterns.

Though soft lithography has numerous advantages, it has several drawbacks when it comes to the fabrication of biomedical microdevices. First of all, the utility of these techniques is often limited by the availability of appropriate masters. $\mu \mathrm{CP}$ works well with only a limited range of surfaces; MMIC is slow; REM, $\mu$ TM, and SAMIM leave a thin film of polymer over the surface. Secondly, the master used to cast the PDMS stamp or mold is fabricated using micro lithographic techniques such as photolithography or micromachining, or from available relief structures such as diffraction gratings or TEM grids. These processes are generally expensive. Therefore, the cost of master mould is high, making soft lithography less desirable for rapid prototyping and small-volume manufacturing. Finally, three-dimensional topography can only be constructed 
through stacking of planary structure. True three-dimensional construction is not possible with soft lithography.

\subsection{Two photon polymerization (2PP)}

Two-photon polymerization is a direct laser writing technique that has gained a lot of research interest because, currently, it is the only technique that is capable of creating complex 3D micro and nano features from polymers using pulsed near infrared laser. As suggested by the name, the resins are polymerized by simultaneously absorbing two photons at red-infrared wavelength.

The principle behind 2PP is simple but exceptionally dominant. First, femtosecond laser pulses are tightly focused onto a liquid resin which is transparent to the infrared. The pulses initiate a chemical process - two-photon polymerization - that converts the liquid resin into a solid. This process is confined to a highly localized area at the focal point due to the nonlinear dependence of the two-photon absorption rate on the laser intensity. Therefore, its spatial resolution is beyond diffraction limit. It has brought the light curable resin into the realm of nanofabrication.

The fabrication process of $2 \mathrm{PP}$ is very similar to laser stereolithography. Stereolithography is a planar process and cannot generate true $3 \mathrm{D}$ features. To generate $3 \mathrm{D}$ features, layers of 2D geometric features are created on different layers and stacked to provide the third dimension. Whereas, in 2PP the laser can be moved three -dimensionally inside a volume of photoresist, therefore, it is a volumetric process. The polymerization occurs along the trace of the focus. This allows the fabrication of any computer-generated 3D structure by direct laser "writing" into the resin. The non-irradiated liquid resin can be dissolved in developer, 
leaving behind the polymerized replica of the computer model. In contrast to conventional photolithography, 2PP offers a genuine 3D volume micro fabrication method.

With this method a feature resolution of $100 \mathrm{~nm}$ or higher can be achieved. Furthermore, due to the nonlinear absorption process, it is possible to directly write inside a given volume ("real" 3D writing), since the polymerization only takes place inside the focus of the laser beam. Therefore, complex 3D-structures can be inscribed into a suitable photosensitive material which is selectively cured. There are two types of photoresists; positive photoresist and negative photoresist. When a positive photoresist is used, the exposed region becomes soluble and eventually washed away by the developer. Negative photoresist operates in the exact opposite manner in which the non-irradiated area is removed by the developer and the exposed region is hardened due to cross-linking of polymer chains. The two main advantages of using a positive photoresist over a negative is the creation of a feature size is four times smaller and also able to produce a three dimensional templates, which are used in various applications in the biomedical industry. $2 \mathrm{PP}$ is professed as the most promising technique for the fabrication of $3 \mathrm{D}$ biomedical microstructures. Many researches have conducted to demonstrate these potentials in biomedical applications [34-37]. Fig 1.8 gives a SEM image of a stent made by this process.

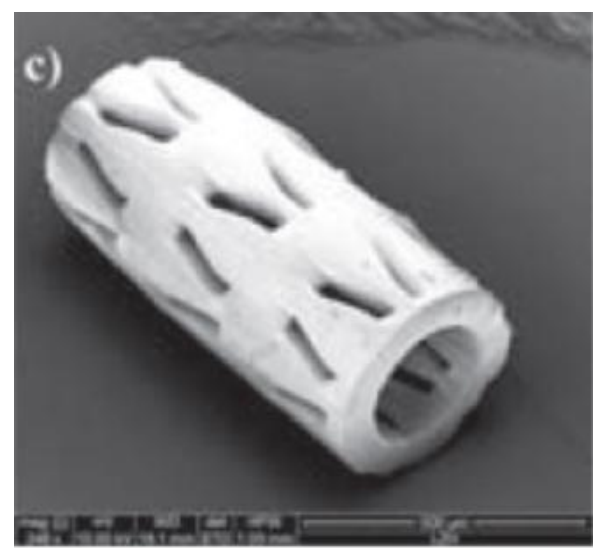

Fig. 1.8 Stent created by 2PP [37]. 
PDMS is not photosensitive; therefore 2PP cannot be applied to PDMS. C. A. Coenjarts et al. [38] illustrated two approaches for creating PDMS based microstructures by two-photon 3D microfabrication via photohydrosilylation and radical-initiated cross-linking. In both approaches, an initiator is added into the uncured PDMS based liquid before irradiation, to make it photosensitive. So far, this is the only study has been conducted on two-photon 3-dimensional microfabrication of PDMS elastomers.

Though a laser direct writing method is a good alternative to lithography to fabricate complex 3D micro/nano-devices, there are some limitations associated with the existing method.

- Using the technology of two-photon polymerization femtosecond laser technique is limited to photosensitive materials.

- $\quad$ The substrate material plays a very important role in the production of nanostructures using two-photon polymerization technique. The reason being is that every substrate material exhibits different properties to light reflectivity. As more light is being reflected upon the fabrication of the nano-structured sample by the laser beam, the less are the chances for the structure to be properly adhered to the surface of the substrate. There is also a possibility that the nano-structure will be fully or partially washed away as soon as the developer is applied.

- Photo initiators (molecules which have low photo dissociation energy) are often added in order to increase the material photosensitivity. 


\subsection{Ablation of PDMS}

Laser machining through ablation is a direct machining method. It is based on the removal of polymer material by using intense UV or infrared radiation provided by laser. Many researchers have proposed methods for creating microstructures ablating solid PDMS [39-40]. Hansong Zeng [41] demonstrated Picoseconds laser ablation of Polydimethylsiloxane (PDMS).The study shows that laser ablation rate per pulse increases with laser fluence and pulse number. The laser-drilled hole diameter increases with pulse number, and it saturates above certain pulse number for low fluence. Maria Graubner et al. [42] studied incubation and ablation behavior of PDMS for 266nm radiation. Silica like material and polycrystalline graphitic carbon with a relatively high bond angle disorder were identified as the main solid ablation products. The surface modification of PDMS by UV radiation in the presence of atmospheric oxygen or by plasmas generated by partial corona discharge [43] was reported previously. However these techniques do not allow structuring of the surface without using mask. Yelena V. White [44] demonstrated single-pulse and high repetition rate femtosecond laser machining of high aspect ratio submicron diameter holes on the surface of fused silica and glass, and laser microstructuring of PDMS material and diamond films grown on a silicon substrate. The sample were rinsed with deionized water and dried with dry nitrogen prior to laser interrogation. The laser beam was focused on the front surface of the samples. Microchannels were machined on the surface of PDMS samples by scanning the samples through the focused laser beam at a constant velocity. The holes produced by single laser pulses exhibit a smaller diameter and a smaller amount of debris on the sample surface. The results indicate that the best quality microchannels are formed when the sample is translated at $1.5 \mathrm{~mm} / \mathrm{s}$ with laser pulse energy of $1.6 \mu \mathrm{J}$. Lower laser energies $(0.4-1.2 \mu \mathrm{J})$ do not produce well-defined channels, while higher 
energies $(2-2.8 \mu \mathrm{J})$ form a large amount of debris on the sample surface. The presence of debris on the surface after laser machining suggests that post-machining sample treatment might be necessary. Nonetheless, to a great extent this laser ablation of solid PDMS rely on a mask or mold fabrication, which is very expensive, time consuming and not feasible for mass production. In addition, they have complicated fabrication stages and require special equipment.

\subsection{PDMS Nanocomposites}

In spite of its wide use and attractive properties, PDMS has some inherent drawbacks such as poor mechanical properties, low tensile strength, and intolerance to organic solvents [45]. A high surface inertness also restricts certain biological applications in microfluidic chips, since it is difficult to immobilize biomolecules on to its surface [46]. Addition of fillers to the polymer matrix can aid in overcoming some of these drawbacks. For example, Wang et al. [47] reported a chitosan-mediated synthesis of gold nanoparticles on PDMS surfaces under mild conditions. A reducing or stabilizing agent, chitosan, was first coated on the PDMS surface. The coated PDMS was then immersed in $\mathrm{HAuCl}_{4}$ solution to induce localized synthesis of gold nanoparticles selectively on the surface. In other reports, reinforced PDMS were used to make optical and microfluidic devices and were also used in other multifunctional applications such as catalysis and gas separation [48-49].

Polymer nanocomposites were developed in the late 1980s in both commercial research organizations and academic laboratories. Polymer nanocomposites are a class of materials that use fillers possessing dimensions on a nanometer scale reinforced into the polymer matrix. They have attracted significant attention by enabling technological developments across diverse areas 
such as optical devices [50], MEMS devices [51], biosensors [52] etc. Since these nanocomposites can be made biodegradable with tremendous stiffness and strength, they find application in the medical field such as blood bags, cardiac devices, and valves as reinforcing biomaterials. It can be also used in medical devices like biocompatible drug delivery system. Based on the dimensions of the nanoscale phase, polymer nanocomposites can be categorized as zero-dimensional (eg.nanoparticles), one-dimensional (eg. nanotubes), two-dimensional (eg. nanocoatings) and three-dimensional (interweaved networks). The presence of these inclusions can bring forth changes in several polymer properties. For example, mechanical and thermal properties of polymer matrices have been enhanced by encapsulating various fillers such as carbon nanotubes, clay, and silica [53].

In synthesizing PDMS nanocomposites, popular approaches include incorporating premade nanoparticles in to a PDMS matrix with the use of a common blending solvent or by an external reducing agent to result in nanoparticles. However, these approaches involve three or more processing steps and require pre synthesized nanoparticles. As a result, these methods lead to an increased processing time and cost which makes them economically unfeasible. To illustrate this point, Jheng et al. [54] reinforced a PDMS mould with high aspect ratio microstructures without damaging the silicon master mold by using a gas-liquid phase sol-gel process. The PDMS mould with microstructures is peeled off the silicon master mold and swelled by using tetraethoxy silane (TEOS). The PDMS mould is then taken out, cleaned, and treated with $\mathrm{H}_{2} \mathrm{O}$ (the reactant) and aminomethyl propanol (AMP-95; the catalyst) vapors that permeate into the PDMS network. Permeation of $\mathrm{H}_{2} \mathrm{O}$ and AMP-95 vapors into the PDMS network pre-soaked with the TEOS not only allows the in-situ precipitation of the $\mathrm{SiO}_{2}$ 
nanoparticles inside the PDMS network but also prevents the formation of nanoparticles at the surface of the microstructure. Other alternative techniques have also been explored to synthesize PDMS nanocomposite. These synthesize techniques involves multiple steps and longer processing time.

\subsection{Research Motivation}

In summary, PDMS is one of the most commonly used materials in the polymer devices fabrication due to its beneficial properties. Motivated by such diverse applications possible through pure PDMS and reinforced PDMS, numerous efforts have been directed towards developing novel fabrication techniques. But there are some limitations associated with the existing methods such as master creation and replication. Masters are expensive to create. The time and expense involved in generating masks are significant barriers to the rapid prototyping and small volume manufacturing. These barriers have also limited the development of soft lithography, and inhibited the use of micro fabrication in a number of areas. Using the technology of two-photon polymerization femtosecond laser technique, only photosensitive materials can be worked upon. This method is not applicable to PDMS. Construction of 3D PDMS microstructures relies on stacking of 2D thin PDMS bases. True 3D PDMS structuring is currently not available. This has acted as motivation behind this research work and the work presented in this dissertation focused on fabricating pure PDMS and reinforced PDMS devices using laser technology to offer an alternative fabrication route that will potentially lead to a new way for prototyping of PDMS devices. 


\subsection{Research Objectives}

This thesis attempts to establish femtosecond laser micromachining as a direct, flexible and single-step process for efficient production of PDMS microstructures. Femtosecond laser is capable of localized rapid heating within the focal volume, owning to its extremely short pulse duration. This characteristic has been used to structuring solids in micro or nanoscale. If laser parameters are carefully controlled, femtosecond laser may be able to process PDMS resin in micron scale. New range of laser parameters such as $\mathrm{MHz}$ pulse frequency, high peak intensity and high peak power may give new possibility of fabrication of PDMS micro and nanostructures.

Therefore, the main objectives of this research are to investigate femtosecond laser irradiation of PDMS resin and establish PDMS rapid fabrication methods. Generally, laser material processing is intensity-dependent. Small intensity variations can produce vast changes in the structure of the machined surface. Similarly, this investigation will be carried out in different regimes of laser intensity: below and above ablation threshold values. This research attempts to utilize the extreme short pulse character of femtosecond laser to demonstrate direct construction of PDMS microstructures and nanostructures.

It is noteworthy that even though this research is motivated by PDMS's vast applications in biomedical microdevices, there is no intension to venture into biomedical application in this work. Rather, this research will mainly focus on developing an alternative technique for PDMS processing. The potential applications of the developed alternative technique will be explored by future investigations. 


\section{CHAPTER 2}

\section{THERMAL CURING OF PDMS}

\subsection{Curing Mechanism of PDMS}

Curing is that refers to the toughening or hardening of a polymer material by crosslinking of polymer chains, brought about by chemical additives, radiation or heat. Crosslinking generates a 3D molecular network and the polymer remains stiff even if reheated, as the curing involves an irreversible chemical reaction.

An elastomer is required to be cured before it is used for any applications. Silicone elastomers can be easily transformed into a three-dimensional network by crosslinking reaction, which allows the formation of chemical bonds between adjacent chains. The majority of the silicone elastomers are crosslinked according to one of the following reactions.

\subsubsection{Crosslinking with radicals (Peroxide Initiated)}

Peroxide initiated where peroxide is used to produce radicals $\mathrm{R}^{\bullet}$ and initiate bonds between the chains (Fig 2.1) [17-18]. Efficient crosslinking with radicals is only achieved when vinyl groups are present on the polymer chain. This reaction has been used to produce high temperature silicone rubber. The peroxide is added before processing. During cure, some precautions are needed to avoid the formation of voids by the peroxide's volatile residues. Post curing (for several hours in ventilated ovens at elevated temperature) may be necessary to remove these volatiles, which can catalyze depolymerization at high temperatures [55]. 


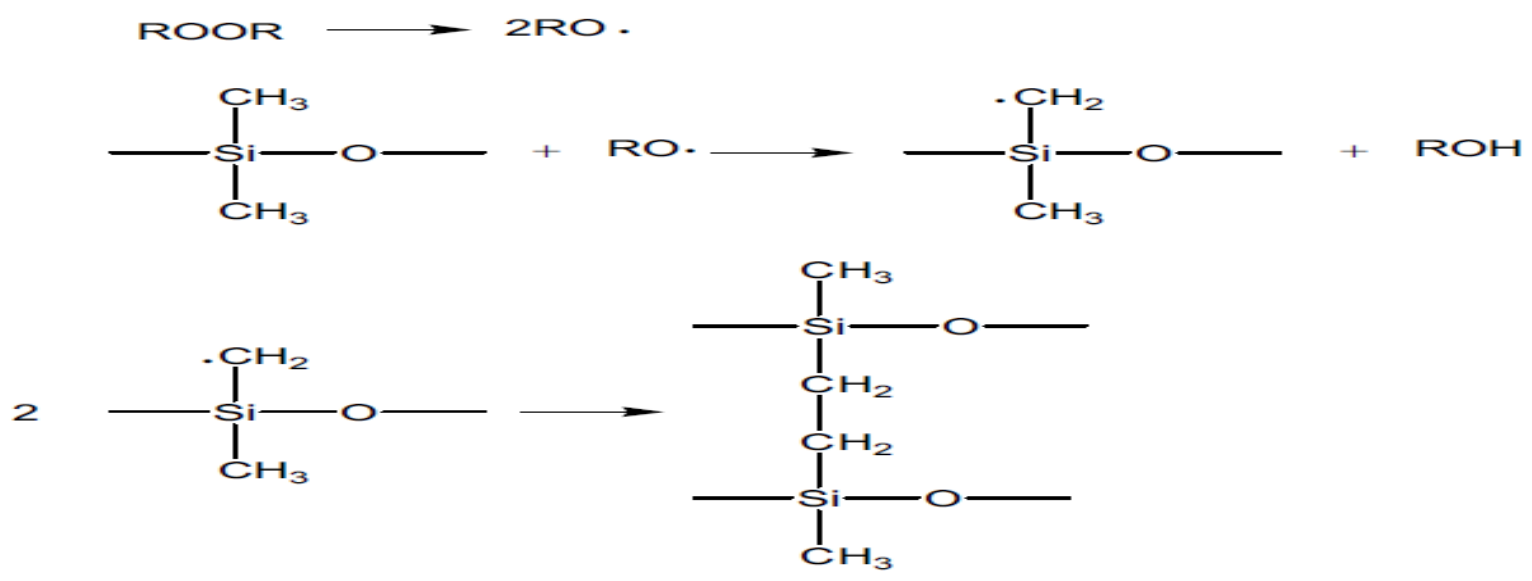

Fig 2.1 Peroxide curing Mechanism for PDMS [55]

\subsubsection{Crosslinking by condensation}

There are two systems for condensation curing: one part system and two part system. One part system crosslinking starts when the product is squeezed from the cartridge or tube and comes in contact with moisture, typically from humidity in the ambient air.

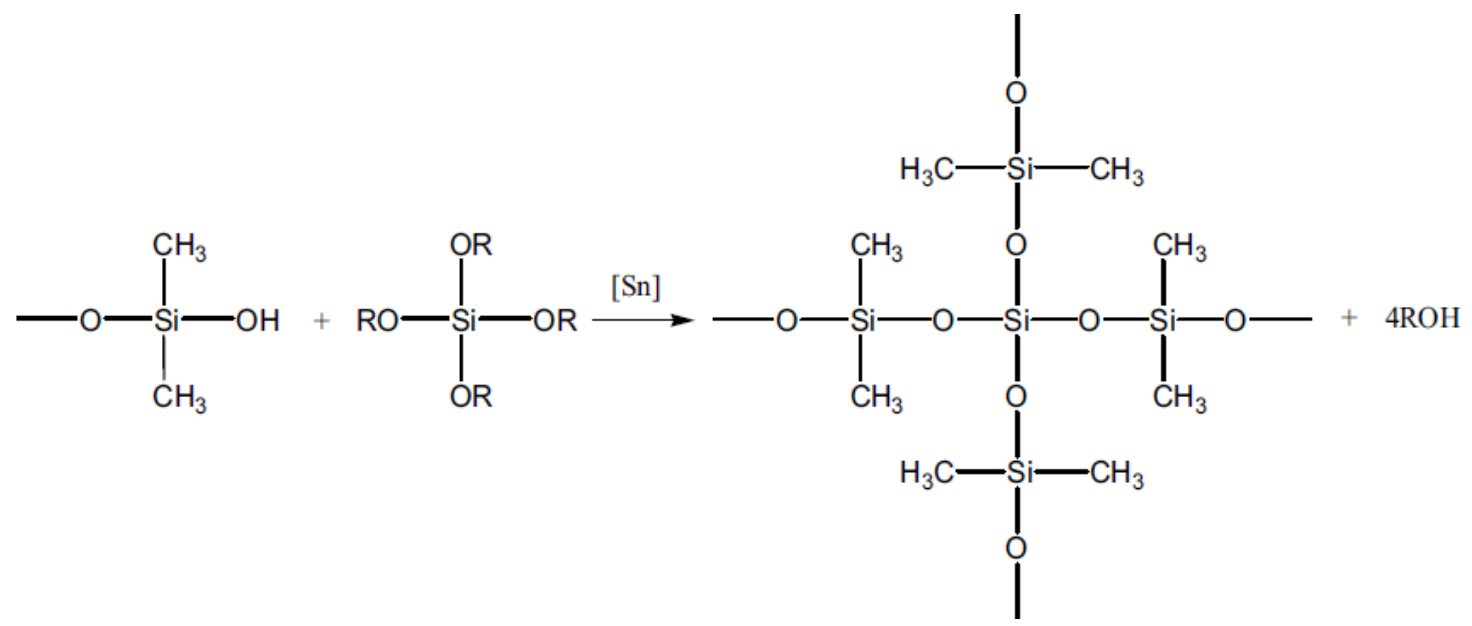

Fig 2.2 Condensation curing mechanism of silicone rubber [55]

This is because the catalyst and cross-linking agent are incorporated in to the base compound at the time of manufacturing. For two part systems, cross-linking starts upon mixing the two components. Alcohol is released as a by-product of the reaction, leading to slight shrinkage upon cure ( 0.5 to $1 \%$ linear shrinkage). Condensation curing takes place at room temperature based on 
the mechanism depicted in Fig 2.2. Silicones with this cure system are therefore not suitable for the fabrication of parts with precise tolerance. The silicone rubbers produced by this method is mainly suitable for sealing, bonding and coating applications due to their unique property [1718].

\subsubsection{Crosslinking by addition}

In room-temperature vulcanization (RTV) method, crosslinked silicone rubber is produced via Si-H group attachment to double bonds. Platinum, palladium, or rhodium salts or their complexes can be used as catalyst. There are no by-products with this reaction. The use of an addition cure reaction for cross-linking eliminates the shrinkage problem. Unlike other methods, RTV do not require additional energy at the time of fabrication, and the PDMS is usually flowable liquid prior to cure. Using platinum-olefin complexes as the catalyst, curing process takes place at room temperature (Fig 2.3).

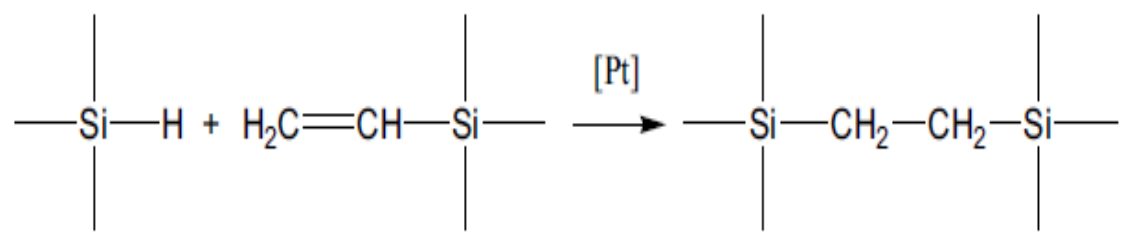

Fig 2.3 Addition curing mechanism of silicone rubber[55]

\subsubsection{Cross-linking by Irradiation}

High energy radiation is also useful method for preparing silicone rubber. This curing is suitable for radiation induced crosslinking of elastomers. Nearly 50 years ago, silicone rubbers are cross-linked by high energy electron beam or gamma rays. Hai Jun Yang et al. demonstrated fabrication and application of high quality poly (dimethylsiloxane) stamps by gamma ray irradiation [56]. D.J.T.Hill et al. reported molecular weight changes and scission and 
crosslinking in poly (dimethylsiloxane) on gamma radiolysis [57]. Much research has been reported on crosslinking of PDMS using E-beam /gamma rays but so-far no studies have been conducted on laser curing of PDMS.

\subsection{Characteristics of conventional curing techniques}

With respect to PDMS device production, the main time-lining step in the typical prototyping process is curing. PDMS cures at room temperature or is heated to speed up the curing process. For Sylgard 184, Dow corning suggests allowing the silicone to cure for approximately $48 \mathrm{hrs}$ at room temperature, $45 \mathrm{mins}$ at $100^{\circ} \mathrm{C}$, or $10 \mathrm{mins}$ at $150^{\circ} \mathrm{C}$ [21]. In the PDMS prototyping literature, researchers have specified a variety of curing temperatures and times as shown in Table 2.1.

In some cases, the PDMS is set to cure overnight out of convenience [21]. In another case; PDMS was cured in 10mins as suggested by Dow corning [21]. Usually no reasons are given for the specified curing times or temperature.

Table 2.1 Curing Parameters for PDMS prototyping (Conventional methods)

\begin{tabular}{cccc}
\hline Grade & Temperature $\left({ }^{\circ} \mathbf{C}\right)$ & Time & Reference \\
\hline Sylgard 184 & 150 & $10 \mathrm{mins}$ & {$[58]$} \\
Sylgard 184 & 150 & $30 \mathrm{mins}$ & {$[59]$} \\
Sylgard 184 & 100 & $1 \mathrm{hr}$ & {$[60]$} \\
Sylgard 184 & Room Temperature & Overnight & {$[61-62]$} \\
Sylgard 184 & 65 & $1-2 \mathrm{hrs}$ & {$[63]$} \\
Sylgard 184 & 65 & $2 \mathrm{hrs}$ & {$[64-65]$} \\
Sylgard 184 & 85 & $2 \mathrm{hrs}$ & {$[66]$} \\
Sylgard 184 & 85 & $3 \mathrm{hrs}$ & {$[67]$} \\
Sylgard 184 & 100 & $3 \mathrm{hrs}$ & {$[68]$} \\
Sylgard 184 & 65 & $4 \mathrm{hrs}$ & {$[67]$} \\
Sylgard 184 & 65 & Overnight & {$[69]$} \\
Not Specified & 80 & $8 \mathrm{hrs}$ & {$[70]$} \\
Silastic J RTV( Dow & 65 & $16 \mathrm{hrs}$ & {$[71]$} \\
Corning) & & & \\
\hline
\end{tabular}


However, it is known that curing temperature affect the amount of shrinkage. Researchers likely to choose a temperature profile for curing based on their time frame for needing the part and past experience in curing devices. For example, if there is no rush and only a few parts are

needed, an overnight or multiple nights cure is a simple way to cure a PDMS part. If multiple parts are needed on a regular basis, the laboratory may have a standard curing temperature protocol.

\subsection{Summary}

From the above description, it is clear that the prevailing techniques used for thermal curing PDMS normally take any time between a few minutes to several hours and even days. Sometimes it may require chemical additives to initiate crosslinking process. 


\section{CHAPTER 3}

\section{DESCRIPTION OF EXPERIMENTAL SETUP}

In this chapter, the material and experimental setups used for this dissertation are presented. All the experiments for this study were conducted using a high power, high repetition rate femtosecond laser in the Micro and Nano Fabrication research facility at Ryerson University, Toronto, Canada.

\subsection{PDMS resin preparation}

All experiments were performed with Sylgard-184, an elastomeric PDMS kit manufactured by Dow Corning. The components for preparing the elastomer were supplied in two parts, base and curing agent. Mixing these in a weight ratio produces the standard product as recommended by Dow Corning.

i) Desired amount (10g) of Sylgard polymer base is measured out in to a clean container.

ii) The elastomer and the curing agent are mixed at a 10part to 1part ratio (10:1). Therefore $1 \mathrm{~g}$ of curing agent is added in to the elastomer.

iii) Due to the base being an extremely viscous substance (3900 cP) [21] similar to honey, thorough mixing in the curing agent is essential to ensure that the reaction between them will occur homogenously and achieve good curing. Hence the elastomer with the curing agent is thoroughly mixed manually for a few minutes. 
iv) During mixing phase, air bubbles appear in the matrix. Bubbles degrade the optical qualities of cured PDMS so it should be removed before curing. Therefore the mixture is set down for about a half hour, or until almost all the bubbles disappear from the solution.

v) After this stage, the PDMS is ready to be cured using the method of choice: this prepolymer will cure at room temperature, or it can be significantly sped up with the addition of heat.

The fabrication process of PDMS using the proposed method is briefly explained in this section. The overall process flow is depicted in Fig 3.1. It involves three steps: spin coating, exposure (laser irradiation) and cleaning.

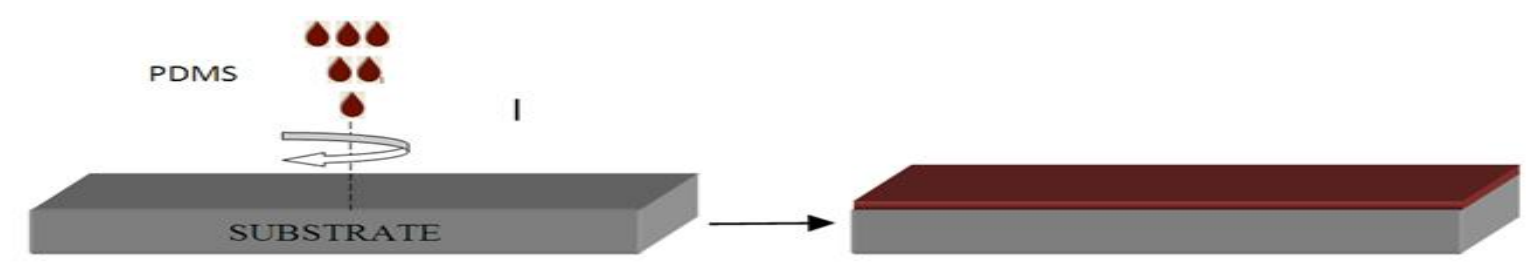

a)

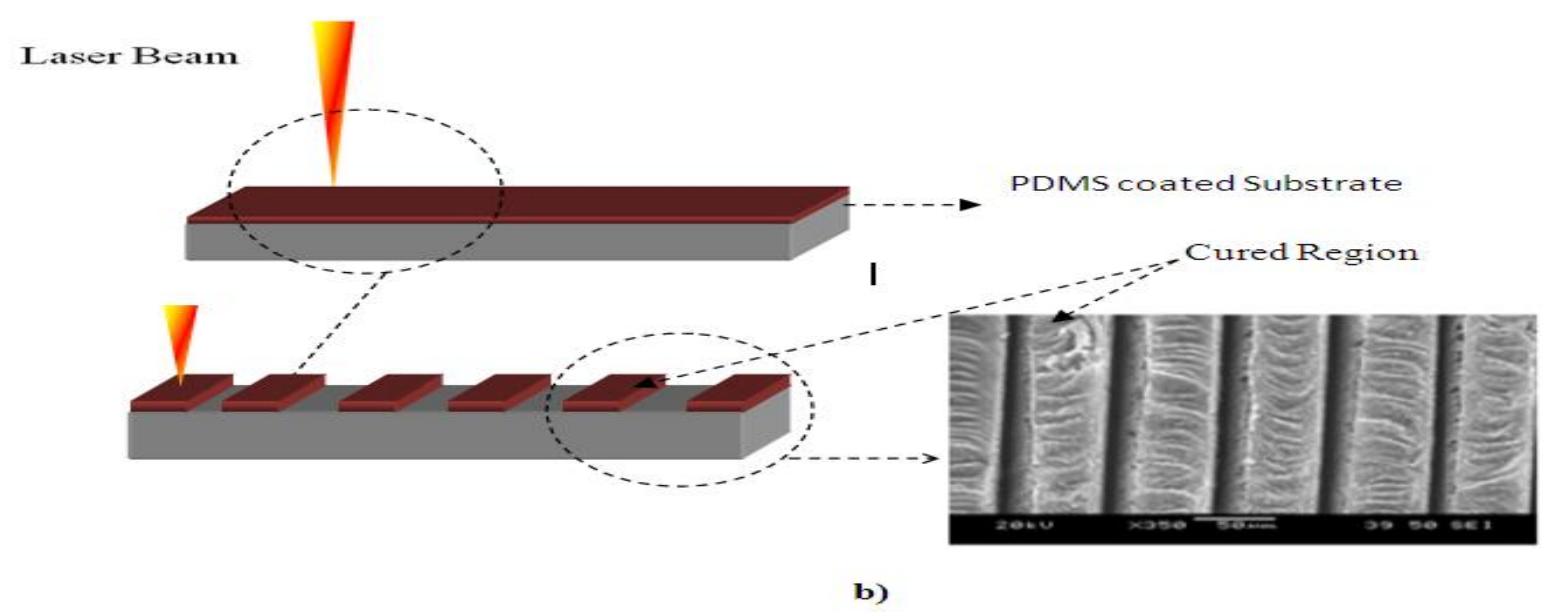

Fig 3.1 Process Flow 


\subsection{Spin Coating}

Spin coating is a procedure used to apply uniform thin films to flat substrates. An excess amount of a solution is placed on the substrate, which is then rotated at a high speed in order to spread the fluid by centrifugal force. To obtain good adhesion, substrate should be cleaned by acetone, followed by air dry. Fig 3.2 depicts a schematic representation of the spin coating process. Two-step spin coating was carried out to achieve desired coating thickness.

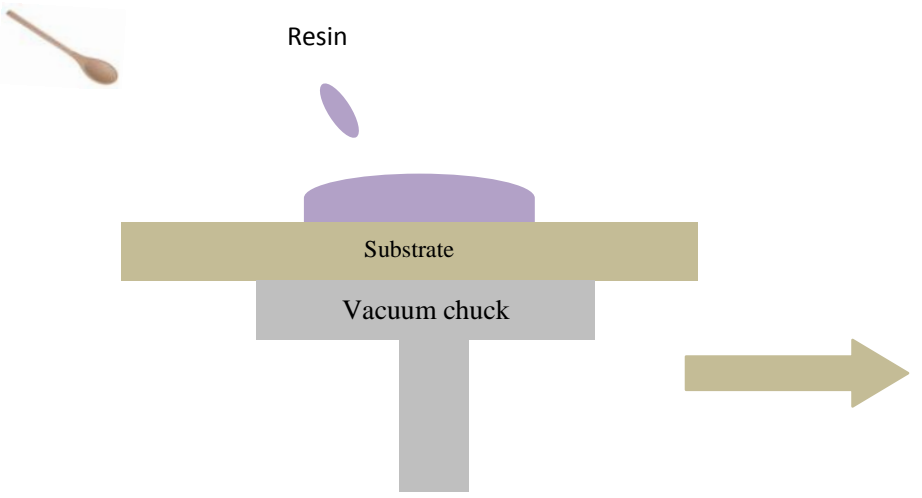

a) PDMS Dispense

Evaporation of Solvent

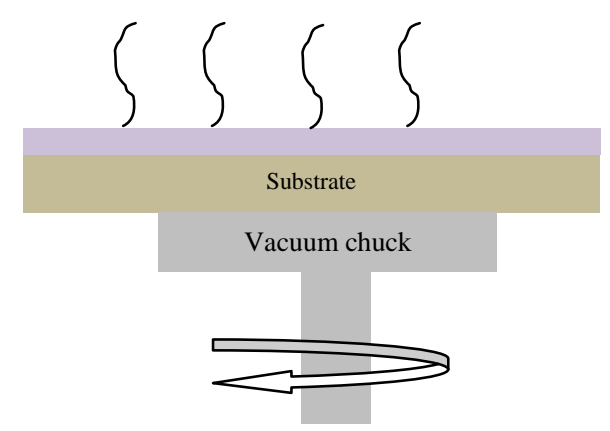

d) Evaporation cycle

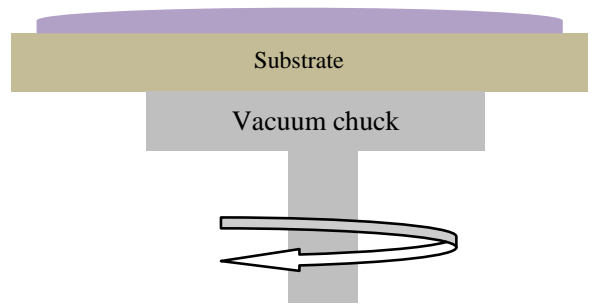

b) Spread Cycle

Fig 3.2 Spin coating of PDMS resin on a substrate a) resin is dispersed on the substrate b) resin is spread evenly by spinning at low speed, low acceleration c) high speed, high acceleration to develop a uniform coating thickness and expelling excess resin and d) evaporating of excessive solvent at final speed to obtain uniform desired final thickness 
First, resin was carefully dispensed in the center of the substrate (Fig3.2 a) and spun for 5seconds at the speed of $500 \mathrm{rpm}$ (Fig3.2b).This step spread the resin evenly by centrifugal force. Immediately after, sample was spun at a spindle speed of $4000 \mathrm{rpm}$ for 60 seconds (Fig3.2 c).This step was used to dispense off excessive resin and to evaporate solvent to achieve desired coating thickness (Fig. 3d).

\subsection{Exposure (Femtosecond laser Irradiation)}

The thin layer of resin was exposed to focused laser beam. The laser beam scans across the substrate surface following specific geometries created by AutoCAD. All the experiments were conducted using an IMPULSE ${ }^{\mathrm{TM}}$ direct-diode-pumped Yb-doped fiber oscillator/amplifier femtosecond laser system capable of delivering a maximum output power of $15 \mathrm{~W}$ at a repetition rate of $26 \mathrm{MHz}$. Available repetition rates are 2, 4, 8, 13 and $26 \mathrm{MHz}$. The laser emits pulses with duration of $210 \mathrm{fs}, 750 \mathrm{fs}, 1300 \mathrm{fs}$ and $10 \mathrm{ps}$ at a central wavelength of $1.03 \mu \mathrm{m}$.

The PDMS resin thin films were irradiated with laser beam of intensity at three different regimes: low, intermediate and high. For the low to intermediate intensity irradiation, optical setup illustrated in Figure 3.3 was employed. The key components include a second harmonic gneratator and a piezoelectric micrometer translation stage. The second harmonic generator is used to convert the central wavelength to $515 \mathrm{~nm}$. While the short wavelength provides better control on the intensity and beam profile, the maximum available laser power is $5 \mathrm{~W}$. For irradiation at higher intensity, 1030nm wavelength was used, providing maximum laser power of 15W. A computer controlled galvoscanner was employed for high intense irradiation of PDMS (Fig 3.5). 


\subsubsection{Setup 1- Low and Intermediate Intense Irradiation of PDMS}

Fig 3.3 is a schematic representation of femtosecond laser setup used for low and intermediate intense irradiation of PDMS coated sample. A plano-convex lens of 500mm focal length and a plano-concave lens of $150 \mathrm{~mm}$ focal length were used to reduce the laser beam diameter and a quarter wave plate is used in between to control the polarization of the laser beam.
1. $1030 \mathrm{~nm}$ Mirror
2. $F=500 \mathrm{~mm}$ Convex Lens
3. $1030 \mathrm{~nm} \mathrm{N/2}$ Waveplate
4. $\mathrm{F}=-150 \mathrm{~mm}$ Concave Lens
5. $515 \mathrm{~nm}$ Harmonic Generator
6. $515 \mathrm{~nm}$ Mirror
7. $515 \mathrm{~nm}$ Mirror
8. $F=-75 \mathrm{~mm}$ Concave Lens

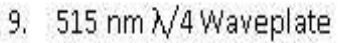

10. $F=300 \mathrm{~mm}$ Convex Lens

11. $515 \mathrm{~nm}$ Mirror

12. Diaphragm

13. Piezo Scanner

14. $\mathrm{EFL}=12,478 \mathrm{~mm}$ Telecentric Lens

15. Sample and Sample Holder

16. Computer controller

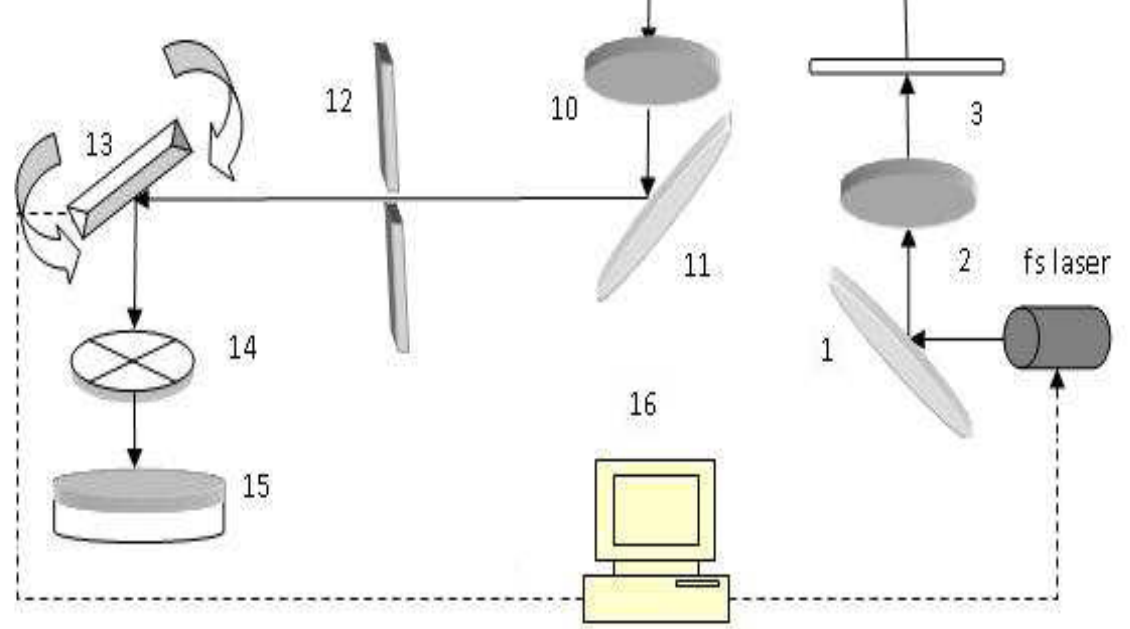

Fig 3.3 Schematic representation of laser setup for low and intermediate irradiation of PDMS

A harmonic generator was used to convert the laser beam to the second harmonics $(515 \mathrm{~nm})$ central wavelength. There are several motivations to use the second harmonic light frequency. In comparison, it is easier to achieve a better curing efficiency and smaller spatial 
resolution. Also shorter wavelength proportionally reduces the diffraction limit of the focal spot and results in smaller feature size. The $515 \mathrm{~nm}$ mirrors were used to dump the 1030nm wavelength out of the beam. Afterwards, a plano-concave lens of $75 \mathrm{~mm}$ focal length and planoconvex lens of $300 \mathrm{~mm}$ focal length were utilized to expand the beam diameter by 4 times to 10 $\mathrm{mm}$. A quarter waveplate was used to rotate the polarization state of the laser beam to circular. In addition, a diaphragm is used to improve the beam profile. Finally, the laser beam was scanned on the substrate surface by a piezo tip/tilt mirror.

The laser spot size, also known as beam waist, is the minimum diameter of the Gaussian beam travelling in a free space. Fig 3.4 shows schematic diagram of the laser spot size and depth of focus for a laser beam converged by telecentric lens.

The telecentric lenses are specially designed to make the focused beam always perpendicular to the flat focal plane regardless of the beam's position in the scan lens. They also offer greater depth of focus and smaller spot sizes when used for laser material processing. Therefore, it is possible to focus the beam on a very thin film of resin without any precision positioning stage. Telecentric scanning lens, by the very nature of their design, maintains a near normal incidence angel of the beam to the work piece. This ensures that position errors and image distortions are minimized over the entire scan field. Having a greater depth of field and smaller spot size are important for precision laser micro-machining processes where there is a need to produce fine spot sizes while maintaining precise placement over a relatively large field. For this study, a telecentric lens with a $12.478 \mathrm{~mm}$ focal length was utilized to focus the laser beam. 
The theoretical laser minimum spot diameter $\left(D_{0}\right)$ was calculated using the following equation [72].

$$
\mathrm{D}_{\mathrm{o}} \approx 1.27 \lambda_{\mathrm{o}} \mathrm{f} / \mathrm{D}
$$

where $f$ is the effective focal length of the telecentric lens, $\lambda_{o}$ is the wavelength of the laser and $D$ denotes the laser beam diameter. Values for these parameters are $12.478 \mathrm{~mm}, 515 \mathrm{~nm}$ and $8 \mathrm{~mm}$, respectively. Hence, the theoretical spot size diameter was calculated to be $1.02 \mu \mathrm{m}$.

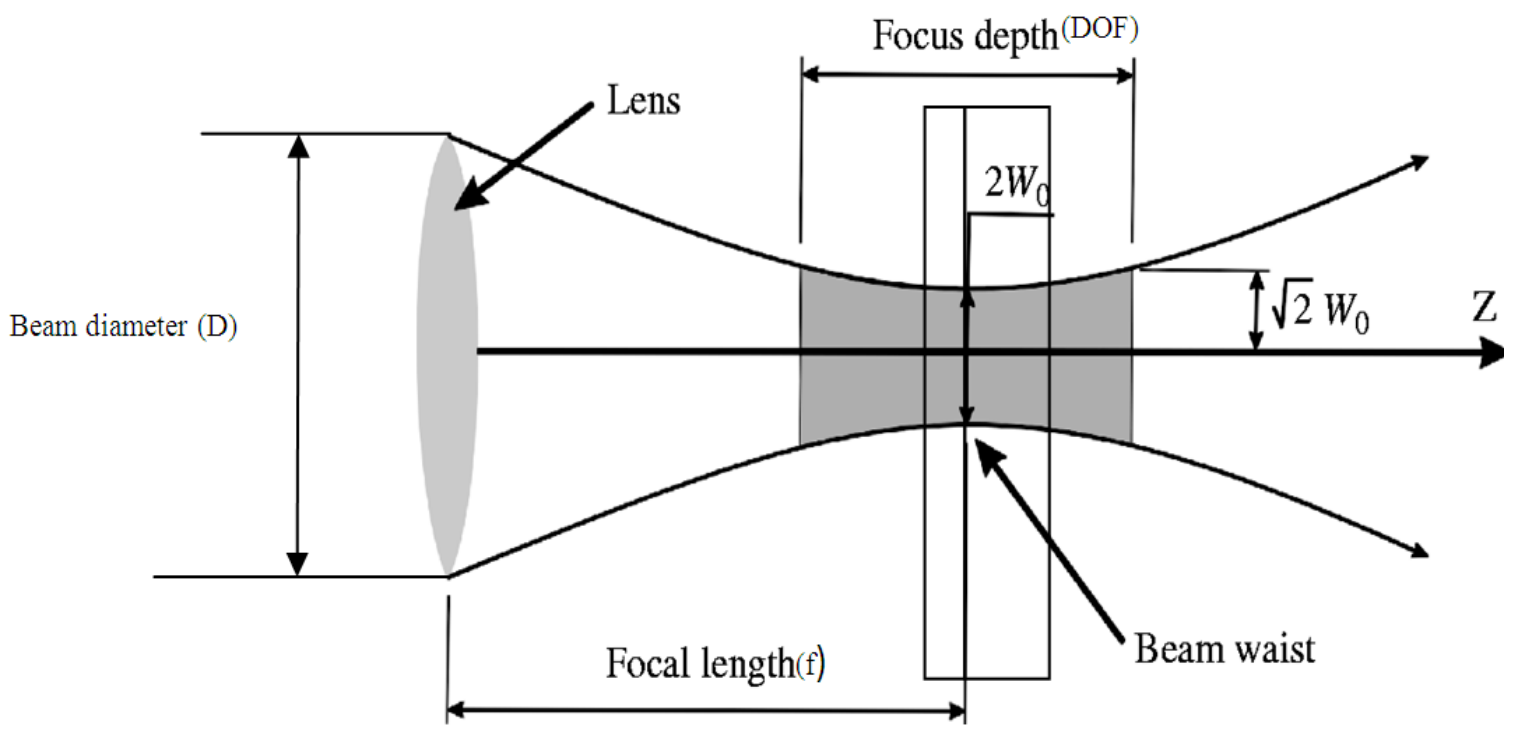

Fig 3.4 Laser spot size and depth of focus for converging laser beam using a telecentric lens

\subsubsection{Setup 2- High Intense Irradiation of PDMS}

Fig 3.5 is a schematic representation of femtosecond laser setup used for high intense irradiation of PDMS coated sample. The previous studies reported a unique growth of fibrous nanoparticle aggregate in different materials by using high power at 1030nm [73-74]. Therefore, laser central wavelength is $1030 \mathrm{~nm}$ with pulse duration of $214 \mathrm{fs}$ was employed in irradiating PDMS to generate hybrid PDMS nanoparticle aggregate. 


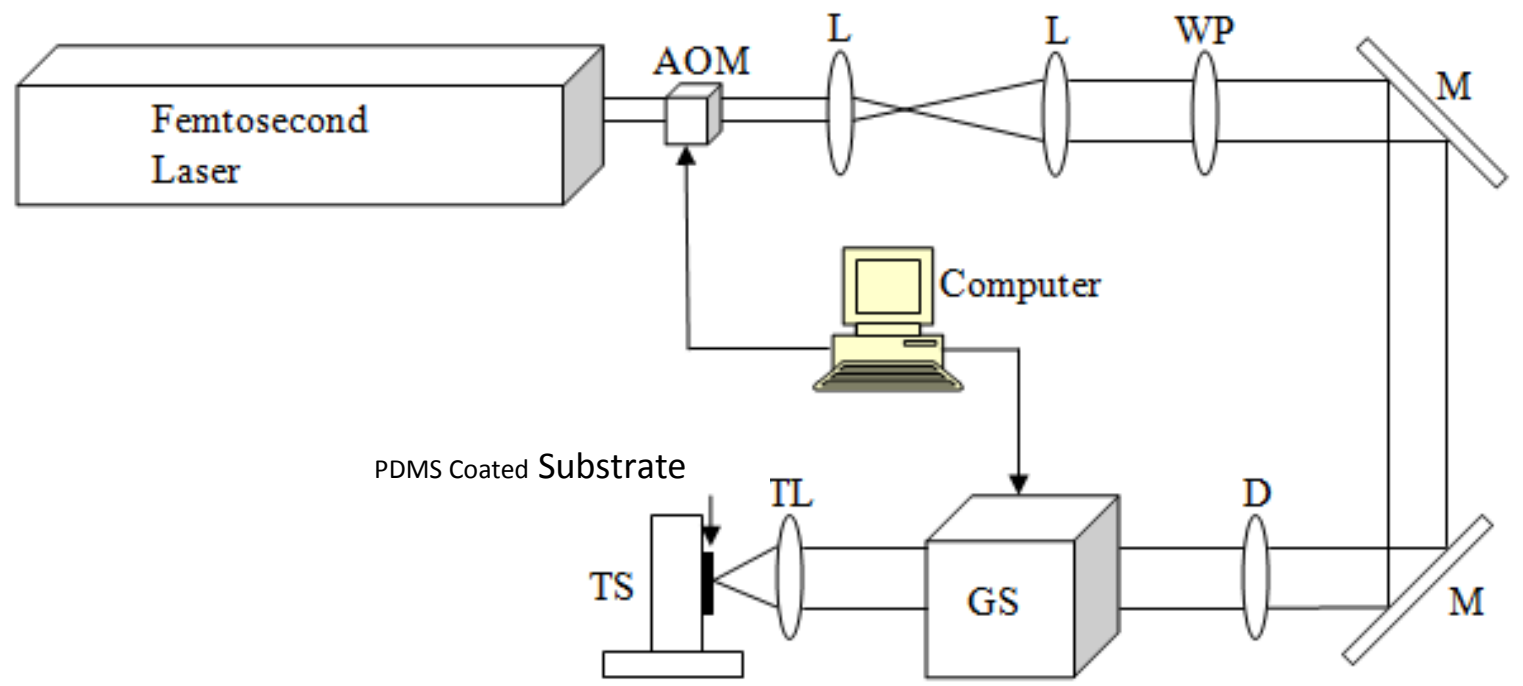

Fig 3.5 Schematic representation of laser setup for High intense irradiation of PDMS : AOM, L, WP, M, D, GS, TL and TS denote acousto-optic modulator, lens, waveplate, mirror, diaphragm, galvoscanner, telecentric lens and translation stage respectively

The laser beam was expanded to $8 \mathrm{~mm}$ in diameter using a combination of UV fused silica plano-convex coated lens of $100 \mathrm{~mm}$ and $200 \mathrm{~mm}$ focal length and focused into a spot of $10.38 \mu \mathrm{m}$ using a telecentric lens of $63.5 \mathrm{~mm}$ effective focal length. A quarter waveplate was aligned with the beam expansion to obtain circular polarization. A galvoscanner was used for high speed beam positioning in XY-axis. An acousto-optic modulator was used, in conjunction with the galvoscanner, to diffract the zero order beams such that the substrate is not machined when the galvoscanner moves from one via to another. A two-axis precision translation stage is used to locate the laser irradiation spot on the sample surface. The pulse repetition rate varied from $2 \mathrm{MHz}$ to $26 \mathrm{MHz}$ internally with computerized selection. All laser power readings were obtained prior to beam entering the galvoscanner. An average power of $15 \mathrm{~W}$ was delivered consistently to the substrate with minimal loss of power in mirrors and lens absorbing energy. 


\subsection{Characterization}

The laser irradiated substrate is immersed in acetone for approximately 5 mins at room temperature to remove unhardened PDMS. The morphology of the formed microstructures is then characterized using a scanning electron microscopy (SEM) (Joel, GSM-6380LV) and an Energy- dispersive x-ray (EDX) analysis. Since the sample is nonconductive, it had to be gold sputtered before observation. 


\section{CHAPTER 4}

\section{IRRADIATION WITH LOW TO INTERMEDIATE}

INTENSITY LASER FLUENCE

The goal of this research is to outline a viable fabricating process for PDMS resin that could be used for rapid fabrication of micro/nano featured components. This chapter describes low and intermediate intense irradiation of PDMS resin using femtosecond laser. The laser spot that used to irradiate the PDMS does not have homogeneous intensity. Instead it follows Gaussian distribution. The laser power should be controlled in the order of $0.01 \mathrm{~W}$ (at $214 \mathrm{fs}$ pulsewidth and $13 \mathrm{MHz}$ ) in order to induce only rapid curing. When the laser power rises above $0.15 \mathrm{~W}$ both curing and ablation will occur. At the periphery of the laser spot, PDMS resin will be cured due to the relatively low intensity. At the center of the laser spot, the intensity is above the ablation threshold, thus evaporate PDMS and create void. The first part of this chapter describes a site-specific curing of PDMS induced by direct low-intensity laser irradiation. The latter part describes the intermediate intense irradiation of PDMS and the fabrication of microfeatures, like pure PDMS microchannels, through laser ablation assisted rapid curing.

\section{1 Low Intense Irradiation: Rapid curing of 2D and 3D PDMS microstructures}

In order to construct 2D and true 3D PDMS structures, the PDMS coated glass substrate is irradiated using low intense femtosecond laser under ambient conditions. The thickness of the glass substrate used is $\sim 1.0 \mathrm{~mm}$. The irradiations are carried out at a pulse width of $214 \mathrm{fs}$ with a scanning speed of $1000 \mu \mathrm{m} / \mathrm{s}$ at $512 \mathrm{~nm}$ wavelength. A very low power $(\sim 0.01 \mathrm{~W})$ is used. Increase of laser power will lead to ablation. The laser beam is either focused on the bottom of 
the resin thin film or inside the resin thin film as shown in Fig 4.1. When the laser beam is focused on the bottom of the thin film, a surface structure is obtained, as shown in Fig 4.2(line width $\sim 15 \mu \mathrm{m})$. On the other hand, when it is focused within the thin film, a free standing structure is created and adhered to the support substrate, as shown in Fig 4.3. The line width is $\sim 20 \mu \mathrm{m}$. These features were generated with a single pass laser scan. The results demonstrate that both $2 \mathrm{D}$ and $3 \mathrm{D}$ microstructures can be generated with the proposed method.

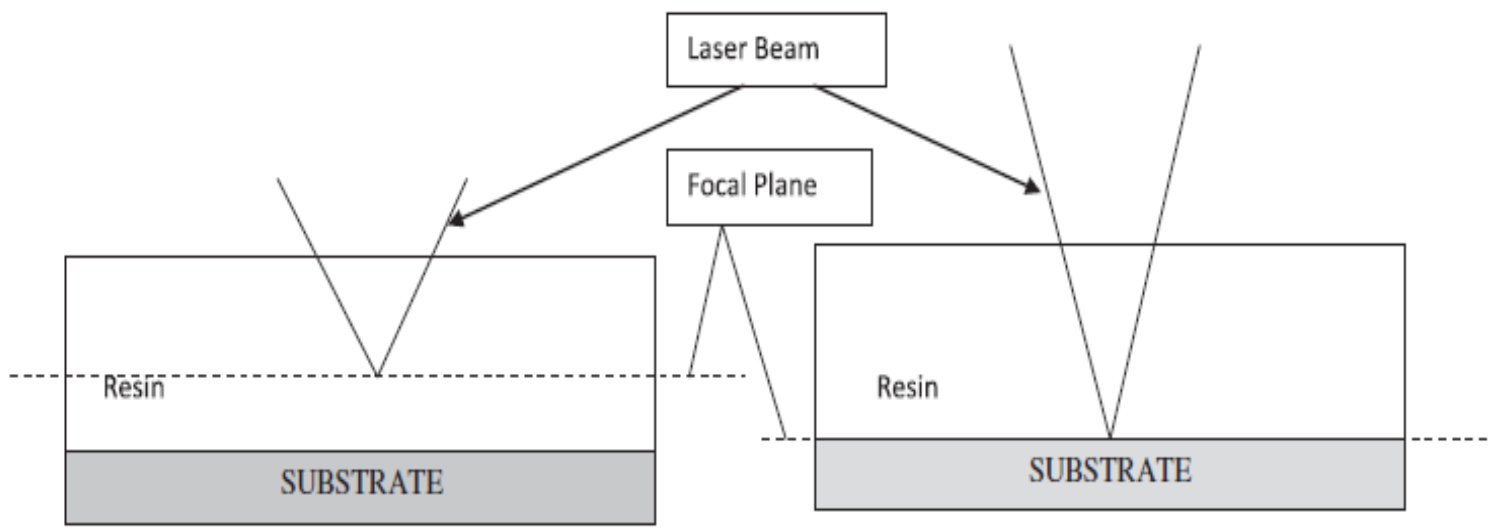

(a)

(b)

Fig 4.1 Position of the focused laser spot a) free standing structure b) surface structure

\subsection{Heat transfer analysis of rapid heating at the volume in vicinity of laser focus}

Rapid curing can be explained by traditional one-dimensional heat transfer model. Upon laser irradiation, part of the absorbed optical energy is instantaneously converted into thermal energy, inducing rapid temperature rise and a chemical reaction that induces polymer solidification. PDMS has very low absorption coefficient for 1032 and 532nm light. The absorption coefficient of PDMS is $\sim 19 \mathrm{~cm}^{-1}$ at $780 \mathrm{~nm}$ [75]. So, only fraction of absorbed laser power is transferred into heat. The resolution of the fabricated microstructures is determined by the size of the smallest solidified volume, called "voxel" (volumetric pixel). Previous study 
shows that short pulse laser results in highly localized heating with a corresponding high peak temperature in vicinity of the focal volume [76]. This localized heating can be future enhanced by repetitive irradiation. When the repetition rate of the laser is sufficiently high (>200 kHz), the interval between successive laser pulses is shorter than the characteristic time of thermal diffusion, leading to heat accumulation in the focal volume. The rapid temperature rise will cure the resin. Since the heated volume is highly confined in vicinity of the focal volume a steep temperature gradients is created surrounding the cured voxel. When the laser irradiation terminates, this cured voxel rapidly cools down, leading to changes in the material properties [77-79]. The size of the voxel increases with increase of laser power as well as with the total number of pulses [76, 79]. With consequence, the femtosecond laser pulses of megahertz pulse frequency serves as a source of heat.

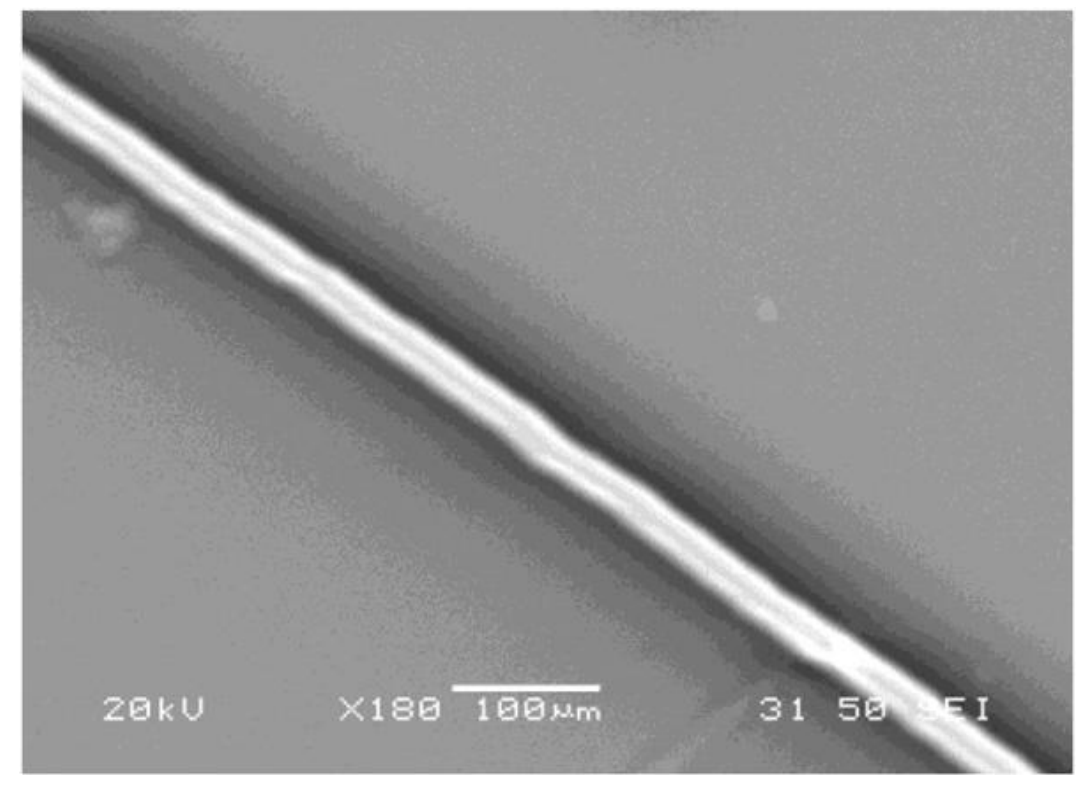

Fig 4.2 Surface Structure 

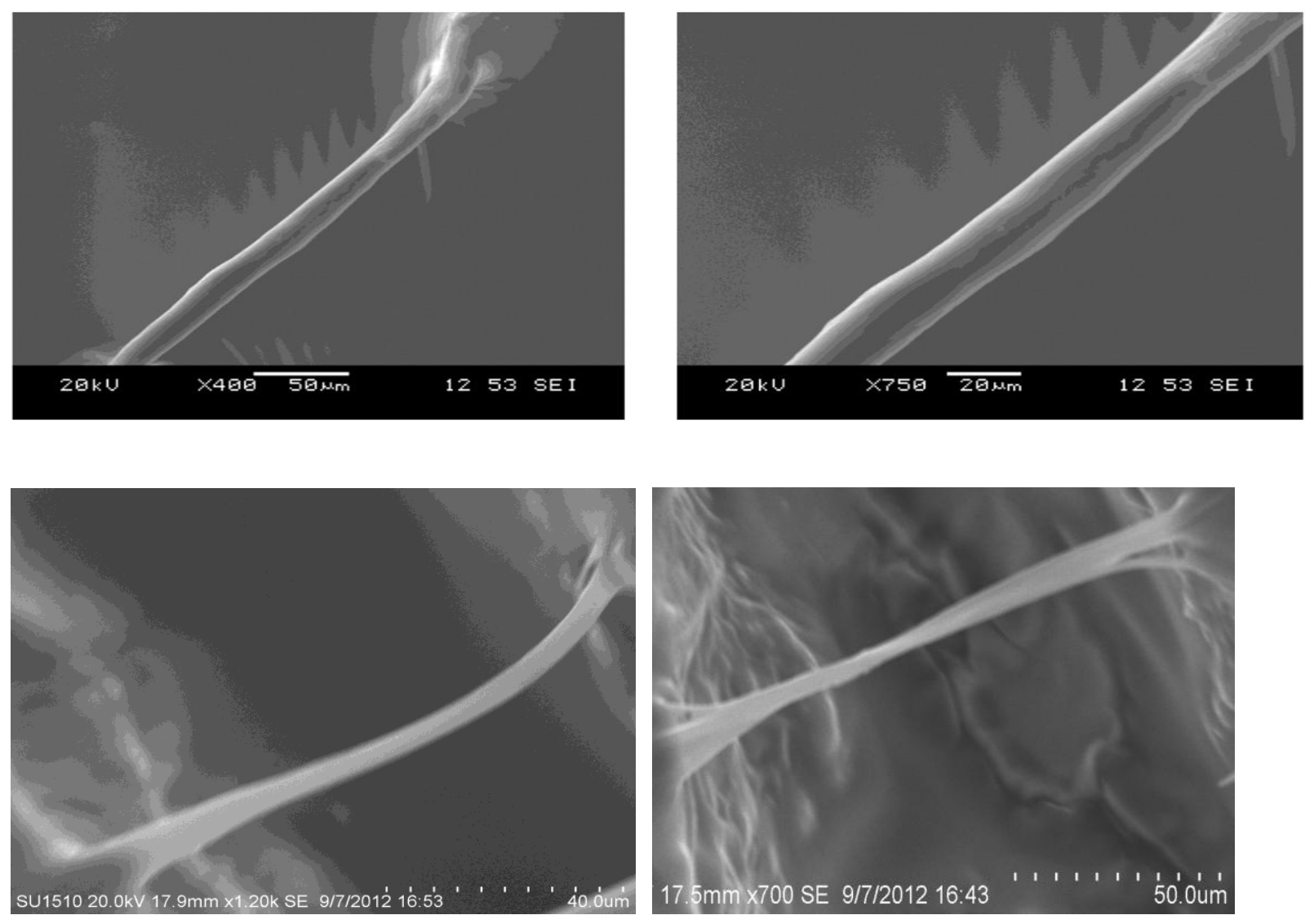

Fig 4.3 Freestanding structures

Considering a femtosecond laser irradiation as a heat source, a numerical model [80] is used to analyze the heat transfer during and after laser irradiation. The incident radiation intensity $I o$, is given by

$$
I_{0}=\frac{4 P}{\pi \times \tau_{p} \times f \times d^{2}}
$$

where $P$ is the laser power, $f$ is the repetition rate of the laser, $\tau_{p}$ is the laser pulse width and $d$ is the laser spot size. The calculated spot is around $1 \mu \mathrm{m}$. However, in practice it is very difficult to maintain a focused laser spot at $1 \mathrm{~m}$ due to defects of optical lenses, turbulence of the 
surrounding atmosphere and temperature fluctuation. From the size of the obtained microfeatures, a more realistic estimation of the spot size would be around $10 \mu \mathrm{m}$. Heat diffusion during laser irradiation is neglected. At $0.01 \mathrm{~W}$ average laser power, the incident radiation intensity is calculated as $1.37 \times 10^{11} \mathrm{~W} / \mathrm{cm}^{2}$ for $214 \mathrm{fs}$. At the center of the laser spot, the surface temperature raise at anytime, $\mathrm{t}$, during the pulse is calculated using equation 4.2 ,

$$
T_{(z, t)}=\frac{I_{0} \times t \times \varepsilon \times \ln 10}{c}\left(2 \times 10^{-\varepsilon(h-z)}\right)
$$

where $h$ is the film thickness, $\varepsilon$ is the material extinction coefficient; $c$ is the volumetric heat capacity and $z$ is the absorbed length along the laser direction ( $Z$ axis). Using the same parameters, the average temperature can be computed using equation 4.3.

$$
T_{a v g}=\frac{I_{0} \times t}{h \times c}\left(1-10^{-\varepsilon h}\right)
$$

The thermo physical properties used in the numerical analysis are given in Table 4.1 [81]. At the center of the Gaussian beam, the surface of the film rises to $4227 \mathrm{~K}$ at the end of the pulse. The average surface temperature is calculated using equation 4.3 and it is found to be $365 \mathrm{~K}$. Thus, at the end of the pulse there is a thin layer of solidified PDMS of very high temperature. After the pulse, the solidified PDMS cools and the dissipated thermal energy penetrates the volume of the liquid resin. Consequently, the solidified PDMS volume grows. The growth comes to an end when the solidified voxel cools down to the cure temperature. Cooling time required to reach the PDMS threshold curing temperature, which is $353 \mathrm{~K}$ at a curing time of 2 hour [109], is assumed as a transient conduction problem and it is calculated using Equation 4.4.

$$
\mathrm{t}=\frac{\rho \cdot V_{c}}{h_{t} A_{s}} \times \ln \left(\frac{T_{i}-T_{\infty}}{T-T_{\infty}}\right)
$$


Where $T_{i}$ is the initial average temperature, $T$ is the threshold curing temperature of PDMS resin, $T_{\infty}$ is the room temperature $\left(\mathrm{T}_{\infty}<\mathrm{T}_{\mathrm{i}}\right), \rho$ is the density of the polymer, $V_{c}$ is the volume of the irradiated area, $h_{t}$ is the heat transfer coefficient $\left[\mathrm{W} / \mathrm{m}^{2} \mathrm{~K}\right], A_{s}$ is the irradiated area. Therefore, the time required to reach curing temperature is calculated as $57.9 \mu \mathrm{s}$.

Table 4.1 Thermo Physical Properties [81]

\begin{tabular}{cc}
\hline Properties & PDMS \\
\hline Density $\left[\mathrm{kg} / \mathrm{m}^{3}\right], \rho$ & 965 \\
Thermal Conductivity $[\mathrm{W} / \mathrm{mK}]$, & 0.15 \\
Heattransfer Coefficient $\left[\mathrm{W} / \mathrm{m}^{2} \mathrm{~K}\right], h_{t}$ & 10 \\
Extinction coeffcient $\left[\mu \mathrm{m}^{-1}\right], \varepsilon$ & 5 \\
Volumetric Heat Capacity $\left(\mathrm{J} / \mathrm{m}^{3} \mathrm{~K}\right), c$ & $1.6 \times 10^{6}$ \\
\hline
\end{tabular}

The resolution of the curing process was investigated by producing individual voxels at a given power. A basic one-dimensional model is attempted to explain the generation of $2 \mathrm{D}$ microstructures through rapid curing. Upon laser irradiation, a small volume in vicinity to laser spot is cured instantly due to the high temperature rise. It is assumed that the diameter of this initial voxel is the same as the diameter of laser spot. The depth of this voxel is determined by the estimated temperature at the center of the laser spot along $\mathrm{Z}$ axis, which is calculated by equation 4.2 and given in Figure 4.4a \& Figure 4.4b. Figure 4.4a shows that at the depth of 0.5 $\mu \mathrm{m}$, the temperature at the center of the laser spot reduces to below the curing temperature of PDMS Since the curing temperature increases with the reduction of curing time, we can expect a 
much higher curing temperature at a curing time scale of micron second. Therefore, the depth of curing is estimated to be no more than $0.5 \mu \mathrm{m}$.

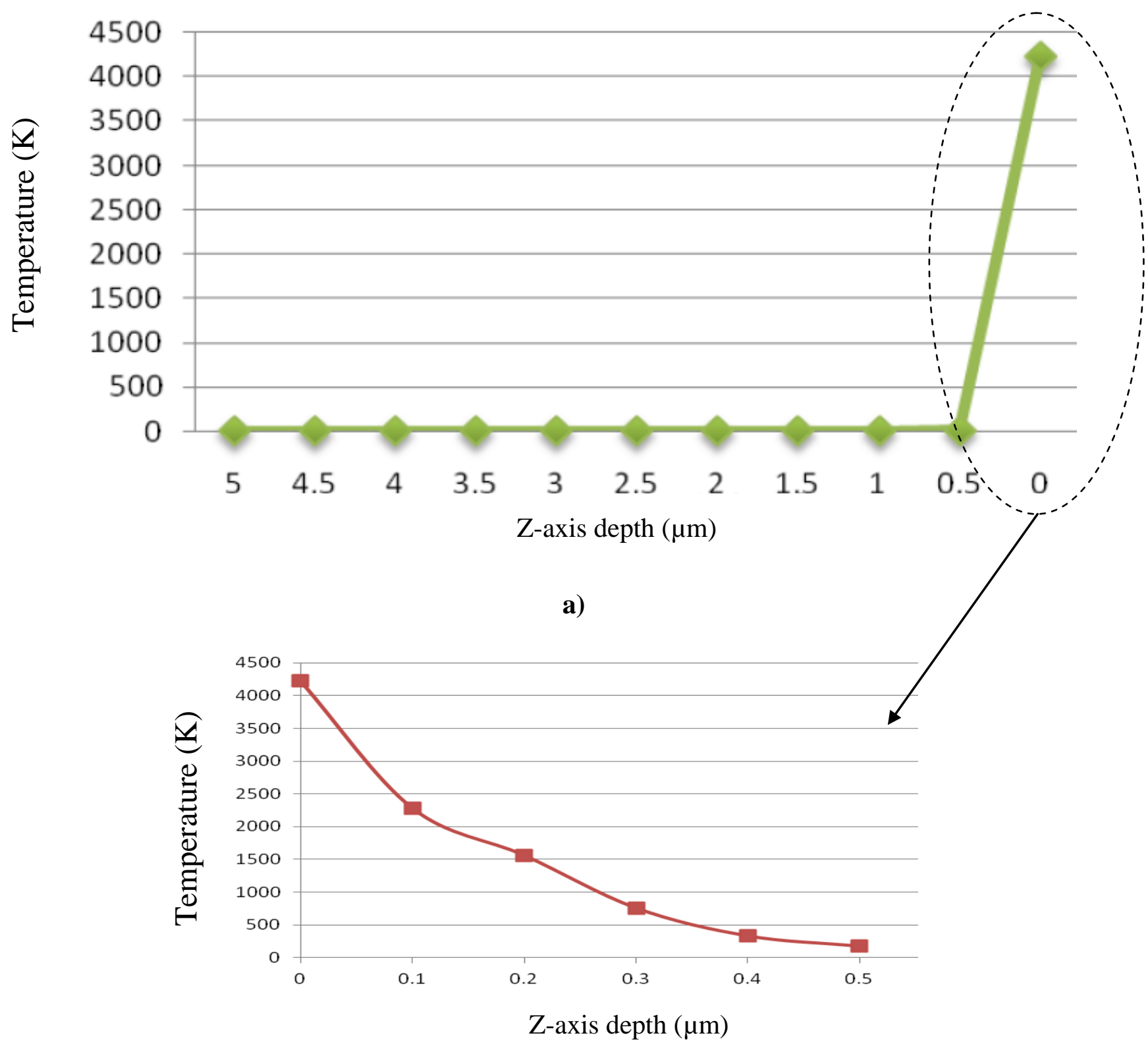

b)

Fig 4.4 a) Z axis depth Vs Temperature at centre of Gaussian laser spot b) Zoomed version 


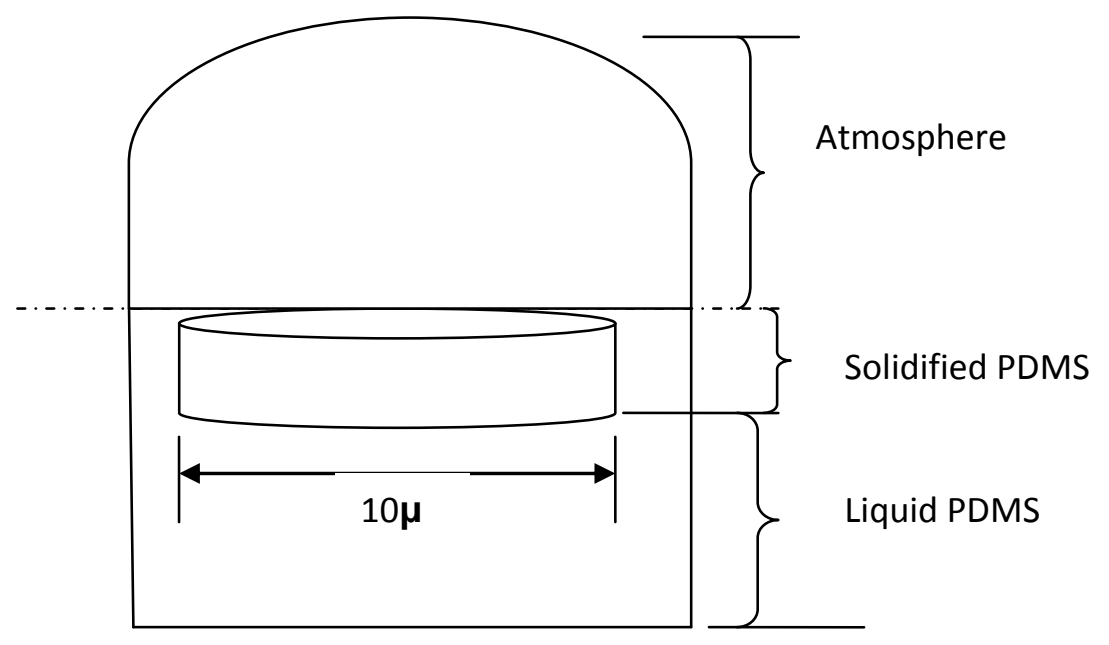

a)

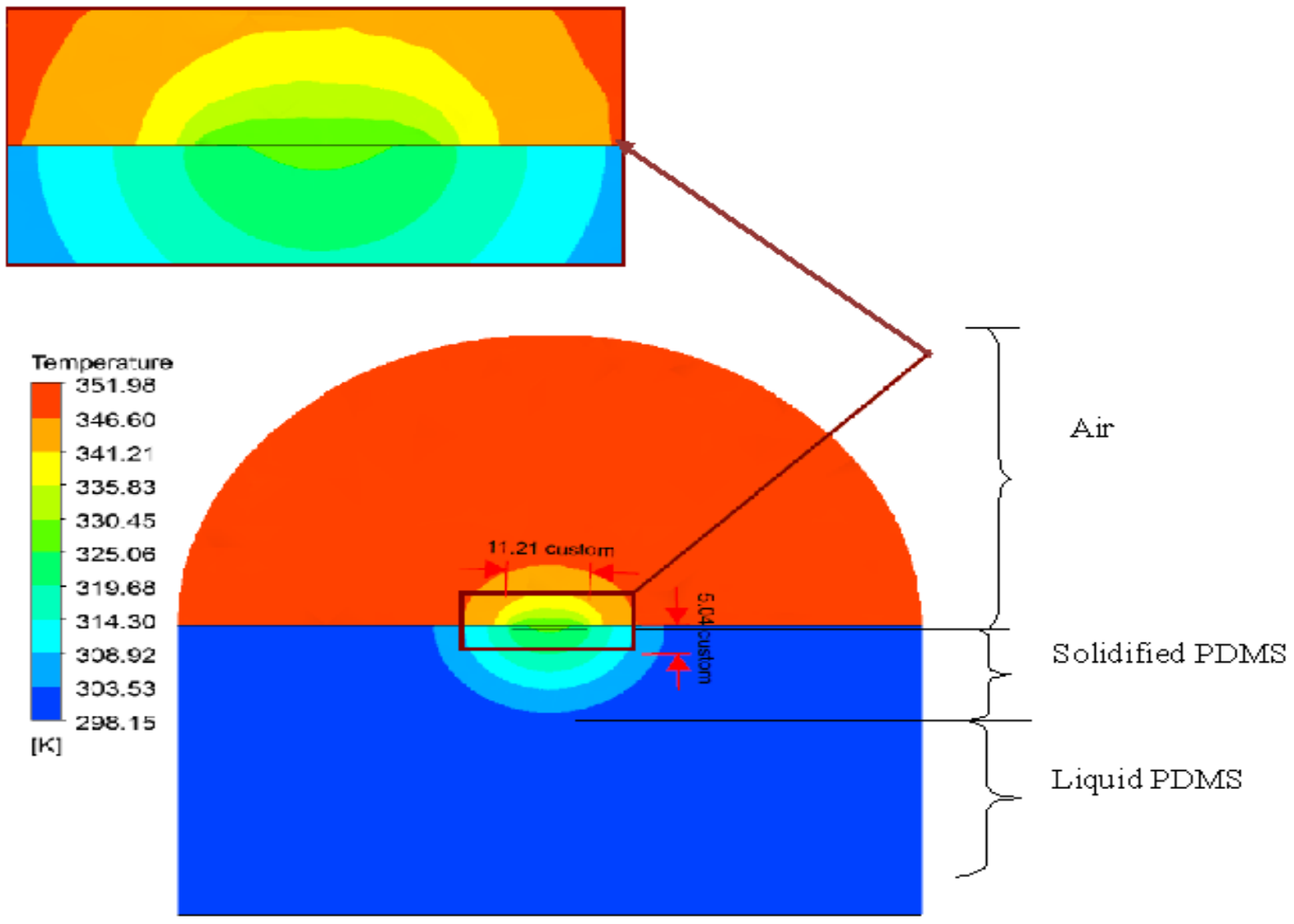

b)

Fig 4.5 a) Simulation Setup b) Simulated Model 
This initial voxel possesses very high average temperature. After the laser irradiation, heat dissipates from this initial voxel to surrounding polymer resin, causing growth of the voxel. An analysis is carried out in ANSYS ICEM CFD (Computational Fluid Dynamics) and ANSYS CFX (general purpose CFD program) to find out the voxel growth and cooling time history. To simplify the analysis, it is assume that the initial voxel is a disc in shape $(10 \mu \mathrm{m}$ in diameter and $0.5 \mu \mathrm{m}$ deep), floating in a liquid PDMS tank. Figure 4.5(a) shows the setup used for simulation with periodic boundary conditions and figure 4.5(b) shows the simulated setup. Model is simulated by treating the disc as a body with homogenous temperature [365K] and the temperature remains homogenous during the cooling. The numerical simulation results show the temperature variation at samples with respect to time. With the passage of time, the temperature decreases and remains constant throughout.

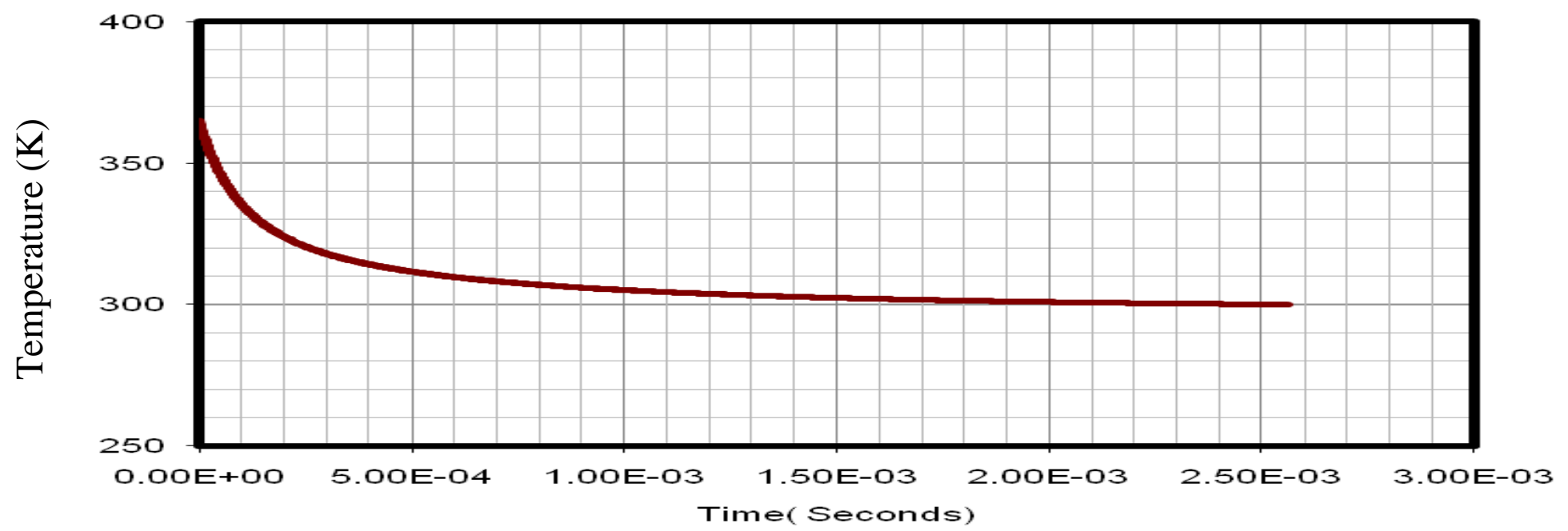

Fig 4.6 Temperature history of polymer resin after the laser irradiation

Figure 4.6 illustrates the graph of temperature versus time and it is evident from the graph that the temperature drops to the curing threshold within $114 \mu \mathrm{s}$, which is comparable to the 
cooling time calculated using equation 4 . From the simulation result, the width and depth of the voxel is found to be $11.21 \mu \mathrm{m}$ and $5.104 \mu \mathrm{m}$, respectively. These numbers cannot be used for a numerical comparison to the experimental results because of the great simplicity of the simulation model, but they demonstrate that feature sizes are in the order of micrometer.

\subsection{Discussions: Strength and degradation of PDMS}

In this work PDMS is cured using femtosecond laser irradiation. So it is necessary to explore the degradation and strength of PDMS. The following theoretical explanation could be used to elucidate the degradation and strength of PDMS.

The thermal diffusion length in PDMS can be calculated using

$$
\mathrm{L}=2 \times \sqrt{ }(\mathrm{k} \tau)
$$

With a curing time $(\tau)$ of $57.93 \mu$ s (calculated using equation 4.4) and a thermal diffusivity (k) of PDMS is $1.0 \times 10^{-7} \mathrm{~m}^{2} \mathrm{~s}^{-1}$ [82], the diffusion length is calculated as $4.81 \mu \mathrm{m}$. Kruczala et al. [83] studied the diffusion coefficients of Nitroxide radicals in Heterophasic propylene-ethylene

copolymers (HPEC). Based on the agglomeration literature data, it is in the range of $4.23 \times 10^{-10}$ to $1.20 \times 10^{-7}$ and the diffusion coefficient of radical's decreases as the temperature increases. Even within $60 \mathrm{~K}$ temperature difference, there is massive decrease in diffusion coefficient. Since the diffusion coefficient of radicals in PDMS polymer is unknown, based on the literature values of other polymers, the radical diffusion length is estimated. The radical diffusion ranges $0.2 \mu \mathrm{m}$ to $7 \mu \mathrm{m}$. In this work, the average temperature is in the range of $4000 \mathrm{~K}$, which paves way for lower radical diffusion coefficient. From the experimental results, it is evident that both 
thermal and radical diffusion plays a critical role in determining the feature size. It shows that with femtosecond laser irradiation, micron sized curing is possible within microsecond time scale.

The thermal degradation of PDMS is determined by temperature and heating rate. According to Thomas and Kendrick [84] the thermal degradation process required activation energy of about $43 \mathrm{kcal} / \mathrm{mol}^{[-1]}$, which defines the degradation into two regimes. When PDMS is exposed to low temperatures $(753-900 \mathrm{~K})$ and a slow rate of heating, the degradation has been shown to proceed via depolymerization of the polysiloxane backbone leading to formation of cyclosiloxanes. At higher temperature regime ( $>900 \mathrm{~K}$ ) and with a faster heating rate, radicals are generated through homolytic Si-C bond cleavage, resulting in bonds reorganization with $\mathrm{CH}_{3}$ radical species and $\mathrm{CH}_{3}$ radical abstracting a $\mathrm{H}$ from the polymer matrix and forming methane. Due to this bonds reorganization, the thermal stability of material increases.

Chenoweth et al. [85] investigated the failure of the polydimethylsilioxane polymer (PDMS) at high temperatures and pressures and in the presence of various additives. It is evident that the degradation initiates at very high temperature about $2800 \mathrm{~K}$ when it is heated at the rate of $266 \mathrm{~K} / \mathrm{ps}$. During the laser curing, the peak temperature at the hottest spot of the irradiated area reaches above 4000K. However, the calculated average temperature obtained by inducing femtosecond laser pulses is about $365 \mathrm{~K}$ which is lower than the degradation temperature $[2800 \mathrm{~K}]$. Also, PDMS is cured in a timescale shorter than a second using femtosecond laser pulses. Rapid cooling follows the curing, limits the decomposition of PDMS. Miao Liu [86] reported the heating effect on mechanical properties of PDMS. At high 
temperature, longer heating time lowers the ultimate tensile stress and young's modulus. In our work, PDMS is cured in timescale of microseconds using femtosecond laser pulses. So there is a prospect to retain the mechanical properties since it is cured in a very short time.

\subsection{Challenges of low intense irradiation of PDMS}

The low intense irradiation of PDMS resin demonstrates the ability of femtosecond laser to generate 2D and true 3D PDMS microstructures through rapid curing. These microstructures are generated using very low laser intensity in the order of $10^{11} \mathrm{~W} / \mathrm{cm}^{2}$. Since an amplified system is used for this study, it is very difficult to maintain at such a low laser power with a reasonable stability. At $0.01 \mathrm{~W}$, laser power fluctuates significantly, in the range of 50\%-100\%, or even more. Therefore, it is impossible to conduct any meaningful parameter study using the amplified system. In another word, the laser system limits the further exploration of this method. An oscillator, rather than an amplified system should be used for this study. An oscillator usually delivers pulses less than 100 femtosecond at an average power in the order of several hundred mille Watt. One such an example is Mercury produced by PolarOnyx. With mille Watt average output power, the pulse-to-pulse laser intensity (power) fluctuation will be in an acceptable range, which allows for precise tuning of laser power. Therefore, precise sculpture of 2D and true 3D PDMS microstructures is possible.

\subsection{Intermediate Intense Irradiation of PDMS: rapid curing and ablation}

When the laser power rises above $0.01 \mathrm{~W}$, both curing and ablation may occur. At the periphery of the laser spot, PDMS resin will be cured due to the relatively low intensity. At the center of the laser spot, the intensity is above the ablation threshold, thus evaporate PDMS and create void. The resultant is a microchannel. Figure 4.8 shows a SEM image of such a channel. 
The rapid temperature rise will cure the resin. As the energy of the laser beam increases to above the curing threshold, the centre of the laser pulse ablates the polymer and the curing threshold boundary moves to the lower intensity of the Gaussian beam (Fig 4.7). The ablation removes the polymer from the central part while the rapid curing process cures the sides of the produced channel shown in Fig 4.8.

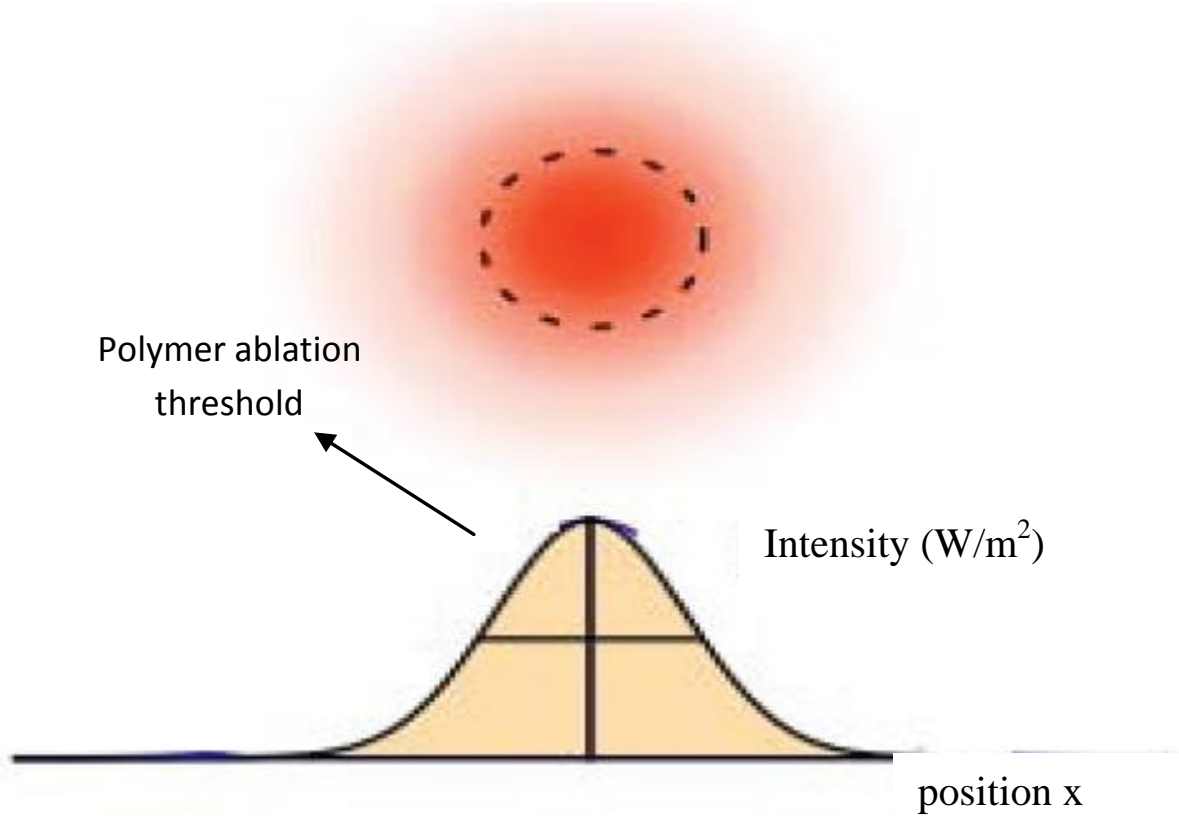

Fig 4.7 Laser intensity profile

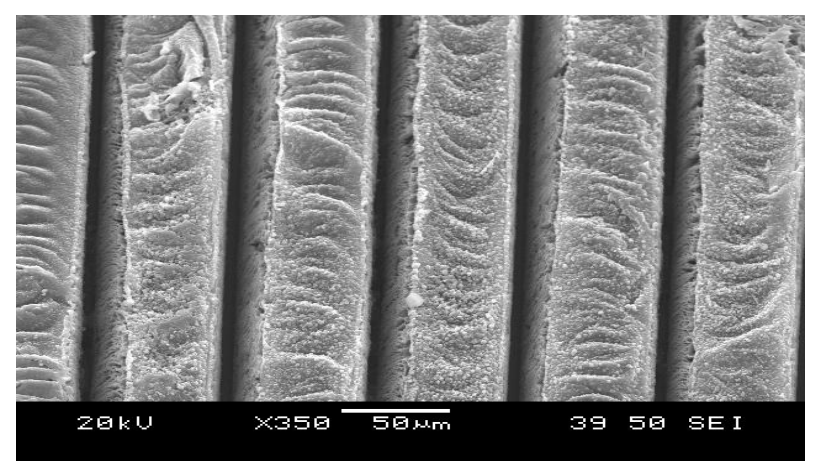

Fig 4.8 PDMS microchannel generated using proposed method at laser power of $0.15 \mathrm{~W}$, pulse width of $214 \mathrm{fs}$ and repetition rate of $13 \mathrm{MHz}$ 


\subsection{Effect of laser power}

To study the effect of laser intensity on the feature size created on rapid curing, micro channels were created at different laser powers. The results is, however, not consistent. For instance in one experiment, which the result is plotted in figure 4.9 , the width of the channel initially, is linearly decreases with laser intensity, and then it reaches to minimum around $4 \mu \mathrm{m}$ at $0.2 \mathrm{~W}$. In another experiment repeated with same parameters, however, the result is quite different. As shown in Figure 4.10, the variation of channel width is random. This study was attempted several times, no conclusive results obtained. At this power level, there are two competing mechanisms. On one hand the low intensity part of the Gaussian beam will cure the PDMS resin and create solid structures, on the other hand the center part of the Gaussian beam vaporizes the PDMS resin and create void. This competition complicates the study of effect of laser power on curing at this power level. As discussed earlier, an oscillator, with low average power and acceptable intensity fluctuation, will be preferred for this study.

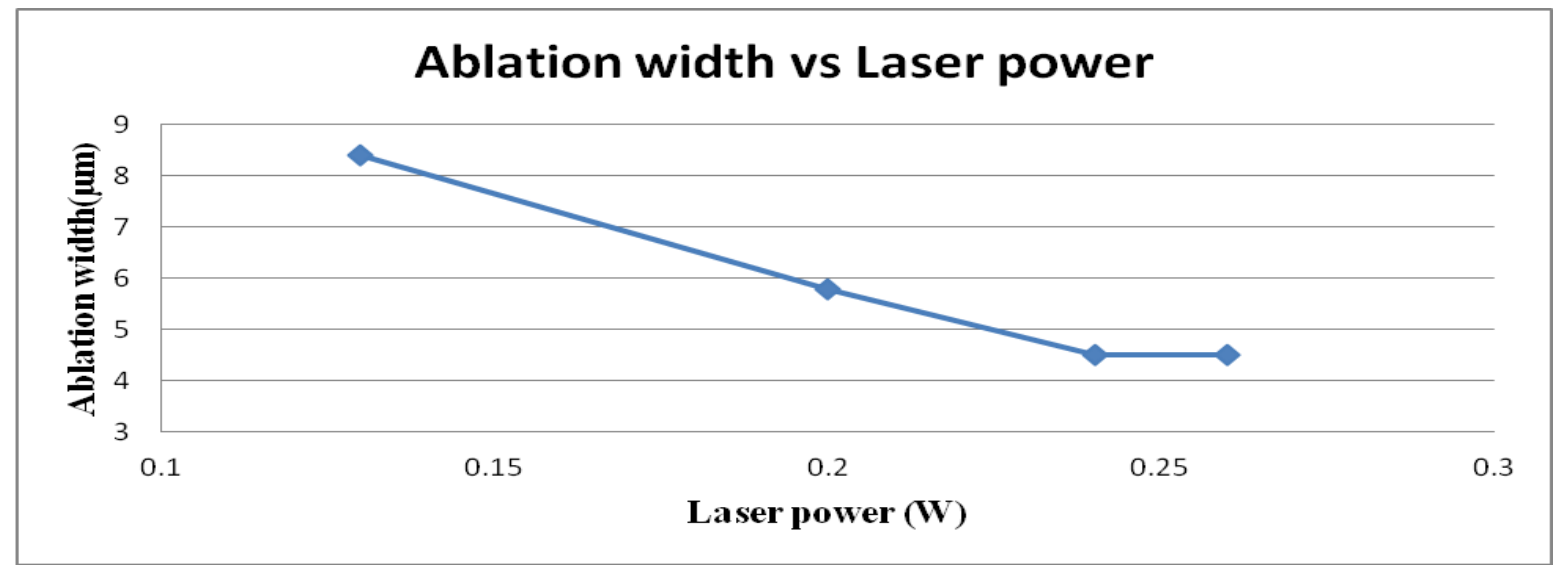

Fig 4.9 Ablation width vs Laser power for $214 \mathrm{fs}$ and $13 \mathrm{MHz}$ 

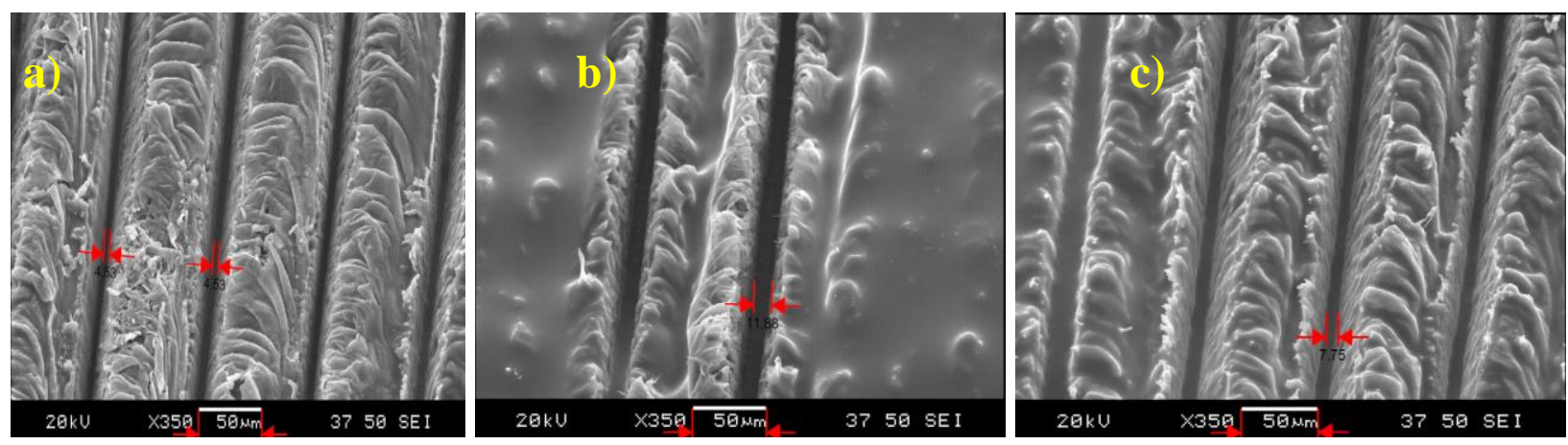

Fig 4.10 Rep rate: 13 MHz, Scanning Speed: $1000 \mu \mathrm{m} / \mathrm{s}$, pulse width $214 \mathrm{fs}$ for different laser power a] $0.24 \mathrm{~W}, \mathrm{~b}] 0.13 \mathrm{~W}, \mathrm{c}] 0.19 \mathrm{~W}$

\subsection{Effect of pulsewidth}

The influence of the pulse width in the femto- to pico- second time regimes on laser curing is studied to determine optimum pulse width for rapid curing. One way to look at the pulse width is the laser-resin interaction time. For the irradiating laser beam, pulsewidth range from $214 \mathrm{fs}$ to $3 \mathrm{ps}$ was used to investigate pulse width effect on the curing efficiency. It is found that the pulse width has a great effect on the curing.
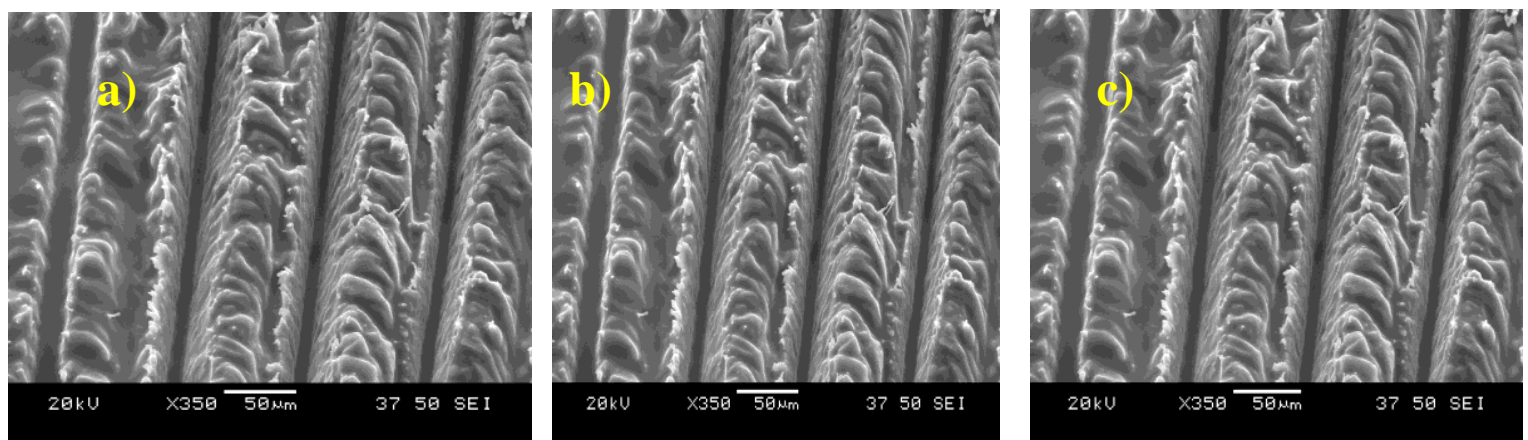

Fig 4.11 Rep rate: $13 \mathrm{MHz}$, Scanning Speed: $1000 \mu \mathrm{m} / \mathrm{s}$, pulse width $214 \mathrm{fs}$ for different laser power a] $0.15 \mathrm{~W}, \mathrm{~b}] 0.16 \mathrm{~W}, \mathrm{c}] 0.2 \mathrm{~W}$ 

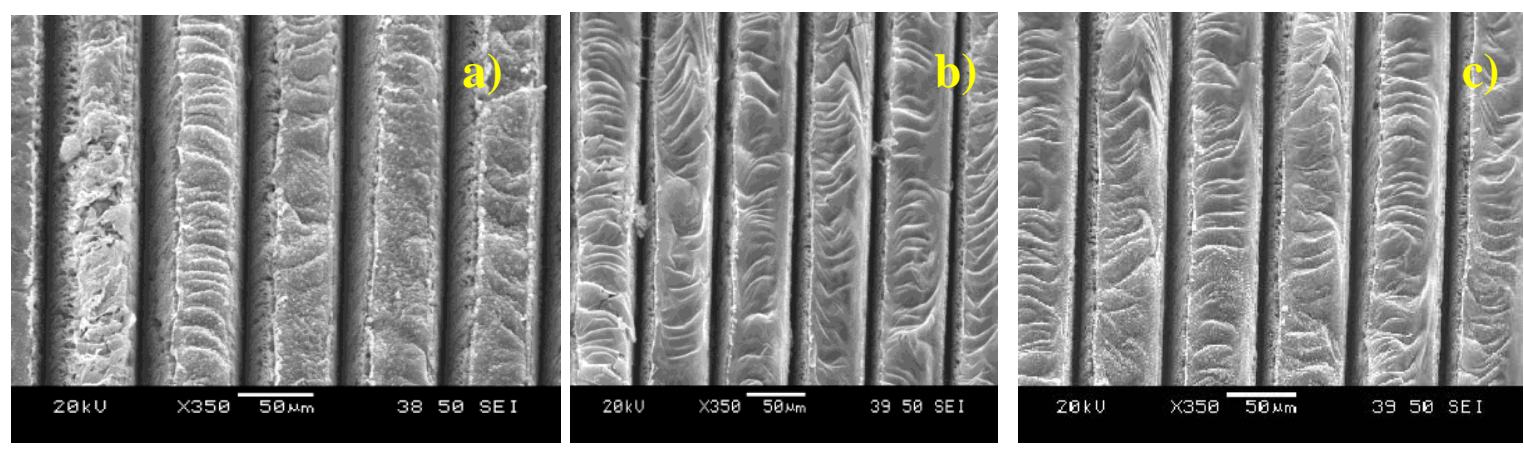

Fig 4.12 Rep rate: $13 \mathrm{MHz}$, Scanning Speed: $1000 \mu \mathrm{m} / \mathrm{s}$, pulse width $714 \mathrm{fs}$ for different laser power a] $0.15 \mathrm{~W}, \mathrm{~b}] 0.16 \mathrm{~W}, \mathrm{c}] 0.2 \mathrm{~W}$
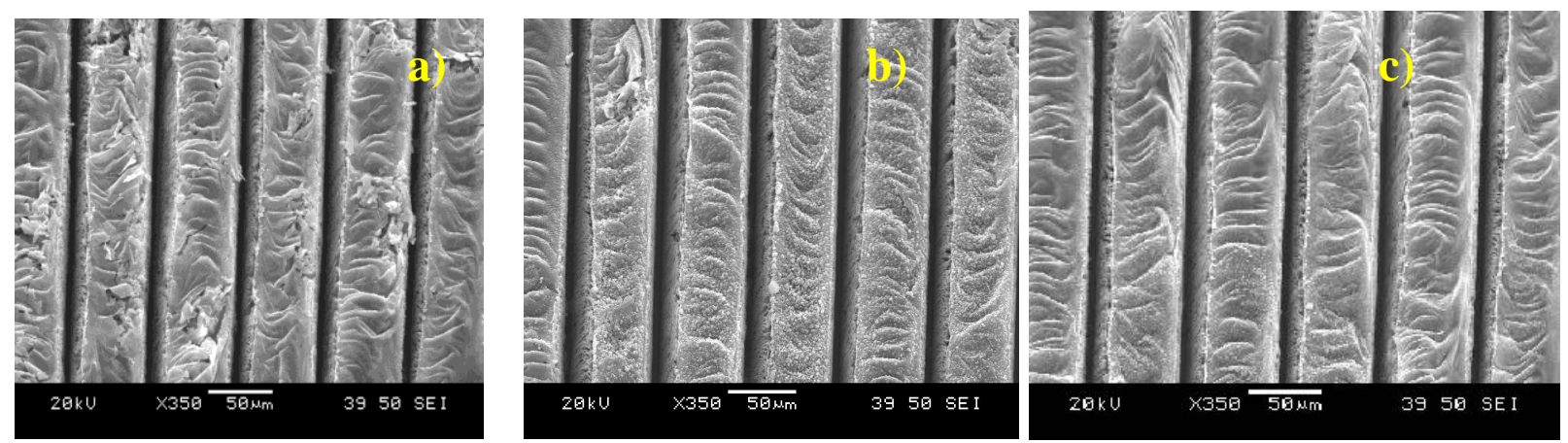

Fig 4.13 Rep rate: $13 \mathrm{MHz}$, Scanning Speed: $1000 \mu \mathrm{m} / \mathrm{s}$, pulse width 1428fs for different laser power a] $0.15 \mathrm{~W}, \mathrm{~b}] 0.16 \mathrm{~W}, \mathrm{c}] 0.2 \mathrm{~W}$

The images show that the formed channel quality improves with the increase of pulse width at $13 \mathrm{MHz}$ repetition rate. Enhanced channel formation is achieved at $1428 \mathrm{fs}$ with $13 \mathrm{MHz}$ and high pulse energy. As shown in Figure 4.11(a) - (c) the sample was irradiated with laser beam of pulse width of $214 \mathrm{fs}$. It is noted that in this time regime, curing efficiency is poor even at higher pulse energy. Channels are partially cured. Heat is not sufficient to cure the focal volume. Curing quality has improved at 714 fs [Fig 4.12(a) - (c)]. Curing quality is further enhanced at 1.5ps [Fig 4.13(a)-(c)] due to extended laser-matter interaction time. Beyond 3ps, there is no initiation of crosslinking, even at extended exposure time. 
The average surface temperature at the end of each pulse irradiation, as indicated by Equation 4.3, is proportional to the intensity of the laser pulse. At longer pulse width, the intensity is lower. At one point the intensity will be too low to generate sufficient surface temperature to overcome the threshold curing temperature. This could explain why at $3 \mathrm{ps}$ polymerization cannot be initiated.

On the other hand, in the regime where the pulse width provides sufficient peak intensity to overcome the curing threshold, the diffusion length of each incoming laser pulse influences the curing quality. As shown in Equation 4.5, the diffusion length of laser $214 \mathrm{fs}$ pulse will has a diffusion length of $30 \mathrm{~nm}$. At 1428fs, that increases to $76 \mathrm{~nm}$. A longer diffusion length means the volume in vicinity of laser focal spot is heated more efficiently, therefore a more uniformed voxel, thus, better quality of created microstructures.

Again, limited by the specifications of the amplified system used for this study, in-depth analysis of effects of laser parameters is not practicable. However, preliminary experimental results obtained with available laser parameters indicate that the size of the cured microstructures could be controlled by adjusting laser power and pulse width.

\subsection{Summary}

Realizing the true potential of PDMS micro devices, many researchers have developed numerous techniques to fabricate PDMS microstructures and devices. However, most of these techniques require masks and also involves multiple steps and long processing time. A new fabrication technique for the formation of pure PDMS microstructures was presented in this chapter. The extreme short pulse duration of femtosecond laser is used to find a new way of rapid curing of PDMS in micron scale directly from resin. 2D and 3D PDMS microstructures are 
demonstrated using the proposed method. In principle, complex geometry also can be achieved using this technique. The laser intensity should be controlled in the order of $10^{11} \mathrm{~W} / \mathrm{cm}^{2}$ in order to induce only rapid curing but not ablation. This indicates that oscillators which emit pulse with mille Watt average power will be more suitable for this task than the amplified system used in this research.

Laser intensity fluctuates significantly at $0.01 \mathrm{~W}$. It is impossible to conduct any meaning parameter studying. However, this study experimentally validated the concept of using femtosecond laser rapid curing of PDMS resin. To the best of the author's knowledge, this is the first time a sitespecific curing of PDMS induced by direct low-intense laser irradiation is reported.

At laser power of $0.15-0.2 \mathrm{~W}$, both curing and ablation occur simultaneously due to the Gaussian intensity distribution of the focused laser spot. A single-scan will create a microchannel. The sidewalls of these microchannels were used to study the effect of pulse width on rapid curing. It is concluded that long pulse width, in the range of a few picoseconds is the optimal for rapid curing. Rapid curing with laser intensity in this regime was used for the rapid prototyping of PDMS microchannels. 


\section{CHAPTER 5}

\section{REINFORCEMENT OF PDMS WITH NANOFIBERS}

This chapter describes the intermediate intense irradiation of PDMS and the fabrication of self-enclosed reinforced PDMS microchannels, through laser ablation assisted rapid curing. Femtosecond laser is used for both nanofiber generation and the fabrication of nanofiber dispersed PDMS microstructures. The strength of the generated reinforced microstructures is investigated through Nanoindentation analysis.

\section{1 Need for PDMS Reinforcement}

Polydimethylsiloxane (PDMS) is one of the most widely explored siloxane polymers because of their unique physical and chemical properties. The properties of PDMS that make it attractive include its elastomeric nature yielding a Young's modulus of 2.2 $\mathrm{MPa}$, low surface energy of $20 \mathrm{erg} / \mathrm{cm}$, outstanding gas permeability, optically transparent, durable, non-fluorescent, biocompatible, chemically inert, and nontoxic; the last two properties make it well suited for cell culture and tissue engineering applications [87-88]. However, because PDMS elastomers in unfilled state cannot form strain-induced crystallization, their mechanical properties are extremely poor. Even the requirement for stiffness is higher when the material is required to perform in nanoscaled 3-D devices. Therefore reinforcement techniques are used to enhance the polymer properties, and extend their utility in most applications. Great enhancement of mechanical properties could be achieved by blending filler particles into the polymers before cross-linking [89-92]. Reinforced PDMS has great potential in the fabrication of biomedical devices, microelectronics, and photonics devices [93-98]. 


\subsection{Reinforcing Materials}

Polymer nanocomposites are a class of materials that use fillers possessing dimensions on the nanometer scale to reinforce the polymer matrix. Amorphous silica, particularly fumed silica, carbon black, carbon nanotubes, calcium carbonate fillers, inorganic nanoparticles, layered nanoclay, carbon nanotubes are the well knownreinforcing fillers for elastomers.

However, reinforcement of transparent polymer resin with traditional micro fillers is difficult due to the need to maintain optical clarity. Nanofibers offer the highest Young's modulus (stiffness), thermal conductivity, and electrical conductivity without scattering light appreciably because of their smaller diameter. As such, their inclusion as reinforcements in polymers (PDMS) holds tremendous potential for unprecedented PDMS polymer properties thereby preserving polymer transparency. Besides, polymer nanocomposites offer an attractive alternative to microcomposites filled with conventional particulates due to the exceptionally high surface to volume ratio of the reinforcing phase and/or its exceptionally high aspect ratio. Also, development of polymer nanocomposites avoids use of many costly and cumbersome fabrication techniques applied to microfillerreinforced composites.

\subsection{Reinforcing Techniques}

Many efforts have been attempted to build reinforced PDMS channels and devices. Normally, reinforcement of PDMS will involve solution mixing, in situ polymerization and melt intercalation. For instance, Burnside et al. [99] synthesized PDMS silicate nanocomposites using the melt process, which involves delamination of silicate particles in the PDMS matrix followed by cross-linking. Zhang et al. [100] reported in situ synthesis of 
PDMS-gold nanoparticles composite films. Thus PDMS monomer and the curing agent were first mixed in known proportions and then cured at $80^{\circ} \mathrm{C}$ for $90 \mathrm{~min}$. The resulting native PDMS thin films were immersed in $0.5 \% 92(\mathrm{~m} / \mathrm{v}) \mathrm{HAuCl}_{4}$ aqueous solutions and incubated at room temperature. The residual curing agent in the PDMS matrix itself is used to reduce $\mathrm{HAuCl}_{4}$. Apart from the above-mentioned methods, there are several approaches for the reinforcement of the PDMS [101-102], e.g., mixing the nanofillers such as inorganic nanoparticles, layered nanoclay, or carbon nanotubes with the uncured PDMS base, followed by the curing process [103-106]; mixing the hydroxyl-terminated PDMS with a silicon alkoxide such as TEOS, followed by simultaneous curing of the PDMS and the solgel process involving hydrolysis and condensation of the TEOS [107]; copolymerization such as forming a PDMS-urethane copolymer [108].

Finally the process used in synthesizing the nanocomposite and curing PDMS involves multiple steps, longer curing time, and replication, overall requiring from a few hours to a few days. In addition, they have complicated fabrication steps, and require masks, multiple sacrificial layers, which limit the application of PDMS in various applications.

\subsubsection{Bonding Techniques}

Many versions of lithography techniques have been developed to fabricate enclosed channels. However, to a great extent these lithography techniques reply on a mask or mold fabrication, which is very expensive, time consuming and not feasible for rapid prototyping. In addition, they have complicated fabrication steps, and require multiple sacrificial layers. A laser direct write method is a good alternative to lithography, but it still requires substrate bonding to enclose the channels. 
Various techniques have been reported for sealing the channels. After channel fabrication, the microchannels (which are normally open on top), must be sealed to form a enclosed channel; the challenge here is to achieve this seal without altering the dimensions of the channel pattern during the sealing process. This step often is the limitation for high volume fabrication methods. Several bonding techniques have been reported and reviewed here.

\section{a)Thermal Bonding:}

Thermal bonding or thermal fusion is the most widely used approach for sealing microfluidic patterns [109]. A patterned substrate is bonded a blank substrate on top using this method; a clamp or hydraulic press is used to hold the two pieces together. Then the assembly is heated to elevate the temperature around the glass transition temperature ( $\mathrm{Tg}$ ) of the substrate. After a period of time, the temperature is lowered and the bonded microdevice is released from the clamp. This should be mentioned that since the bonding

depends on the intermolecular interactions between the contact polymer layers, this bonding method cannot provide high bonding strength for polymeric substrates, and delamination offer occurs. Moreover, this kind of bonding is performed at a temperature close to $\mathrm{Tg}$, channel deformation is very common, which makes this technique unsuitable for sealing microstructures with very low aspect ratios and small dimensions.

\section{b) Solvent bonding:}

Alternatively, organic solvents that dissolve the polymeric substances can be used for microdevice bonding [110]. A thin layer of solvent is spin coated on a blank substrate and then, patterned substrate is quickly brought in to tight contact with the blank. After a period of time, the solvent partially dissolves the polymer at the contact surface, and the 
flexible polymer chains in both substrates are bonded when external pressure is applied. As a result, strong intermolecular interactions are established between the two substrates.

Unlike thermal bonding, solvent bonding is performed at room temperature and provides high bonding strength; however, the organic solvents used should have moderate solubility in the polymer substrates, and their volatilities should not be high. In addition, the amount of solvent coated on the substrate surface should be carefully controlled; otherwise, excessive polymers may dissolve and flow in to the channels, which can block the channels.

Many channel enclosing methods have been studied, including sealing with fusion bonding[111],adhesive bonding, chemical bonding and depositing materials over sacrificial layers followed by wet etching or thermal decomposition [112] etc. All the aforementioned methods have their own advantages and disadvantages. For instance, the most commonly used thermal bonding requires stringent cleaning and high temperatures, often resulting in channel morphing and weak bonds [113]. The residuals stuck to the channel walls after removal of sacrificial layer may hinder the flow inside the channels [114]. Finally, these enclosing methods require multiple steps; hence, it significantly increases the complexity.

Even though extensive work has been done for fabricating enclosed channels, no work has been done to fabricate reinforced self enclosed channels. 


\subsection{Proposed Method: Fabrication of Reinforced PDMS microstructures}

Stereolithography is, essentially, one method from the family of rapid prototyping technologies that involves the curing or solidification of a liquid photosensitive polymer by applying a laser beam across its surface. A Thermostereolithography (TSTL) apparatus, composed of a femtosecond laser, with a laser beam focused and directed through an optoelectronic system, creating a cured layer, was developed and constructed for research purposes.

\section{4.1 Generation of Nanostructures Using Laser Ablation}

First, nanofibrous structures are formed by laser ablation of silicon target in ambient atmosphere. Arrays of holes were drilled in to the silicon substrate using femtosecond laser of $13 \mathrm{MHz}$ pulse frequency with dwell time of $10 \mathrm{~ms}$ and a power of $15 \mathrm{~W}$. Web-like fibrous nanostructures were formed in and around the drilled hole due to the agglomeration of the bulk quantity of nanoparticles created during laser ablation. It is also found that the pulse frequency at which the particle aggregations form is in agreement with the theoretically calculated critical time to start nanoparticle formation [115]. The fibrous nanostructure formed shows certain degree of self-assembly consisting of rings and bridges. Also, the fibrous nanostructures have relatively uniform diameters $(50 \mathrm{~nm})$ and did not observe a wide range of variation in size distribution [73].Energy dispersive X-ray spectroscopy $[E D X]$ analysis shows that these fibrous structures contain only Silicon with a trace of Oxygen. The mechanism of the particle-aggregate formation can be explained by laser ablation and gas phase condensation. Vapor condensation in the plume of laser ablation generates nanoparticles and they aggregate [73]. This method demonstrates a unique, novel and inexpensive way of creating 3D fibrous nanostructure. Vapor condensation starts with 
nucleation, proceeds with growth of supercritical nucleus and comes to a halt due to the effect of quenching. The observed structure is an aggregate of particles of few tens of nanometers. This indicates that nanoparticle aggregation occurs in the latter part of the vapor condensation by the collision of large sized nucleus. The density of nucleus decreases as the plumes expands. For nanoparticles to aggregate in massive scale and form fibrous nanostructures, a continuous source of vapor needs to be supplied to the expanding plume in order to maintain the nucleus density at a certain level. The plumes generated by consecutive laser pulses must arrive before the critical time. Therefore, the formation of fibrous structure is possible only if the pulse frequency is higher than $1 \mathrm{MHz}$. In the case of silicon, fibrous structure was first observed at repetition rate of $2 \mathrm{MHz}$ and maximized between 8 and $13 \mathrm{MHz}$ [73]. Fig. 5.1 a) shows the process of nanofiber generation.

\subsubsection{Reinforcement of PDMS}

The PDMS mixture is poured over the ablated silicon substrate. The substrate is then spun at a spindle speed of $4000 \mathrm{rpm}$ for $60 \mathrm{sec}$, thus forming reinforced PDMS with nanofiber. Generally, the process used in reinforcement of PDMS involves multiple steps, longer time, and replication, overall requiring from a few hours to a few days. This work, demonstrates a novel technique to reinforce PDMS with nanofiber. To the best of the author's knowledge, this is the first time reinforcement of PDMS induced by direct laser

irradiation is reported. Fig. 5.2 shows the spin coating of ablated silicon substrate with PDMS. 


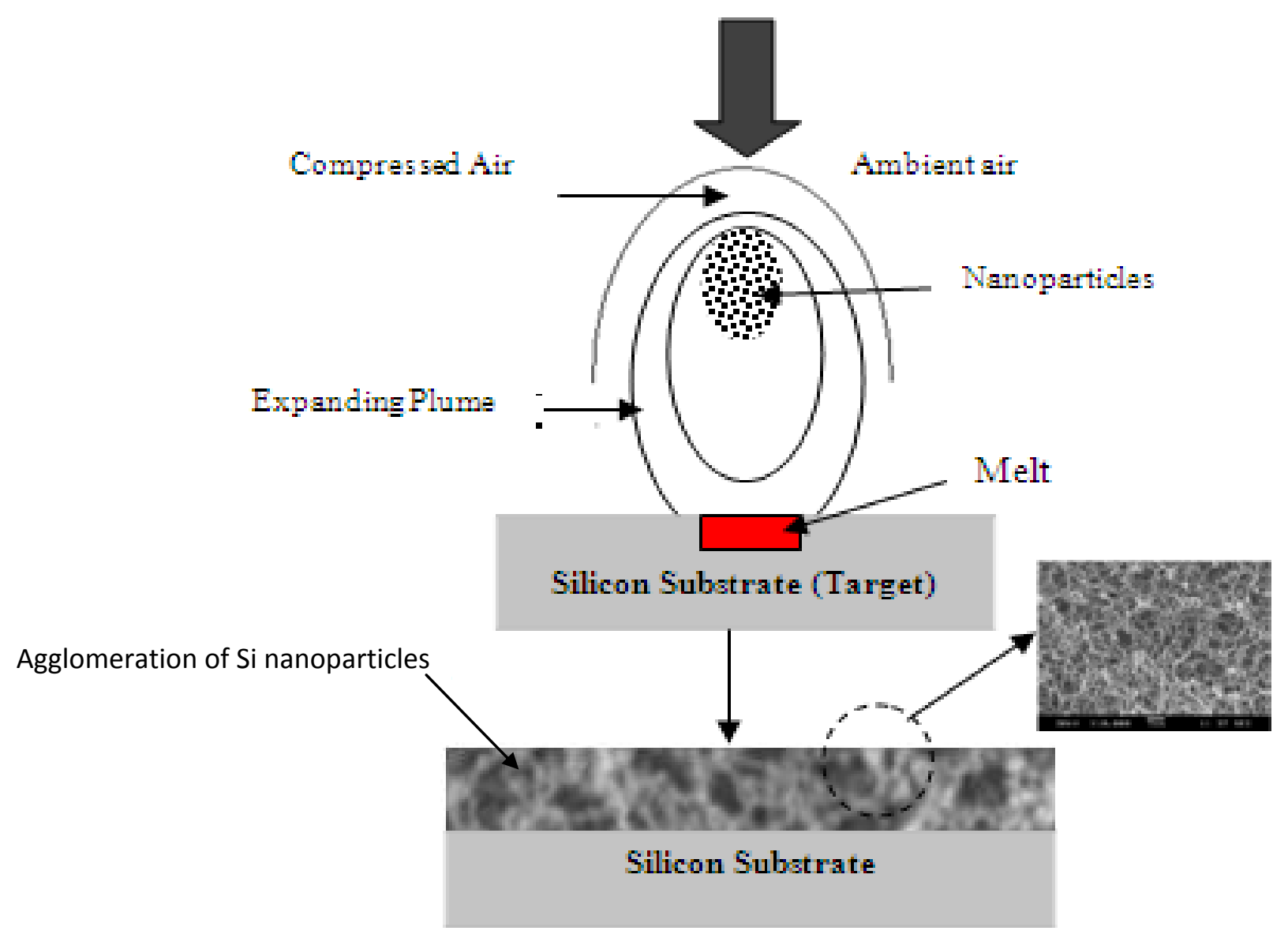

a)

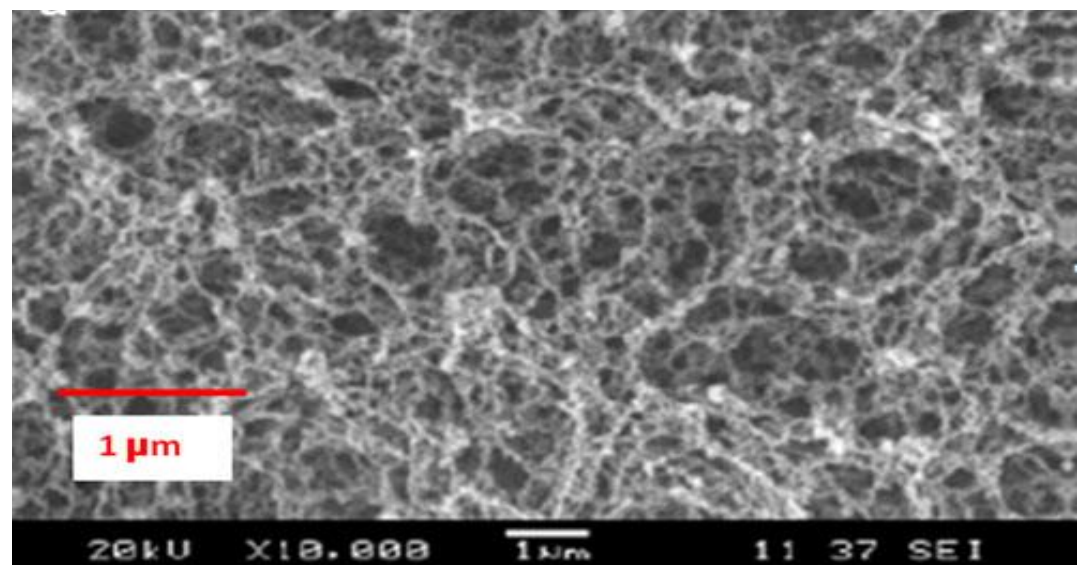

b)

Fig 5.1 a) Generation of nanofibers using femtosecond laser ablation b) SEM image of a nanoparticles aggregate after laser ablation of silicon substrate 
PDMS

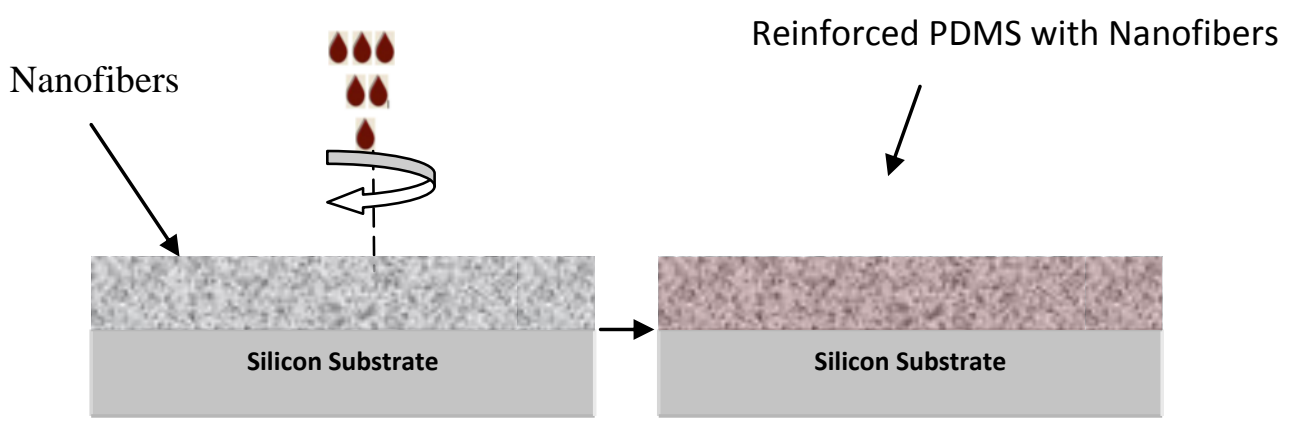

Fig 5.2 Reinforcement of PDMS with silicon nanofibers

\subsubsection{Reinforced Structure Fabrication: Self-enclosed reinforced PDMS Channels}

In order to generate micro features, this PDMS coated ablated silicon substrate is exposed to femtosecond laser irradiation. Fig. 5.3 shows the Laser irradiation of PDMS coated ablated Si substrate. The laser beam was used to scan across the PDMS coated ablated silicon substrate to cure the resin in predetermined patterns. The irradiations are carried out at different pulse widths of $214 \mathrm{fs}, 714 \mathrm{fs}$ and 1.5 ps with a scanning speed of $1000 \mu \mathrm{m} / \mathrm{s}$. Laser power was varied between $0.13 \mathrm{~W}$ to $0.27 \mathrm{~W}$. Pattern is revealed after the exposed substrate is cleaned with acetone. The morphology of the formed microstructures is then characterized using a scanning electron microscope [SEM] and energy-dispersive $\mathrm{x}$ ray $(\mathrm{EDX})$ analysis. When the laser intensity rises above $0.01 \mathrm{~W}$ both curing and ablation will occur. Upon laser irradiation, the absorbed optical energy, inducing rapid temperature rise, consequently, a chemical reaction leads to polymer solidification. The resolution of the fabricated microstructures is determined by the size of the voxel. When the repetition rate of the laser is sufficiently high $(>200 \mathrm{kHz})$, the interval between successive laser pulses is shorter than the characteristic time of thermal diffusion, leading to heat accumulation in the focal volume. The rapid temperature rise will cure the resin. The laser spot that used to irradiate the PDMS does not have homogeneous intensity, instead it follows Gaussian 
distribution. At the periphery of the laser spot, PDMS resin will be cured due to the relatively low intensity. At the center of the laser spot, the intensity is above the ablation threshold, thus evaporate PDMS and create void.

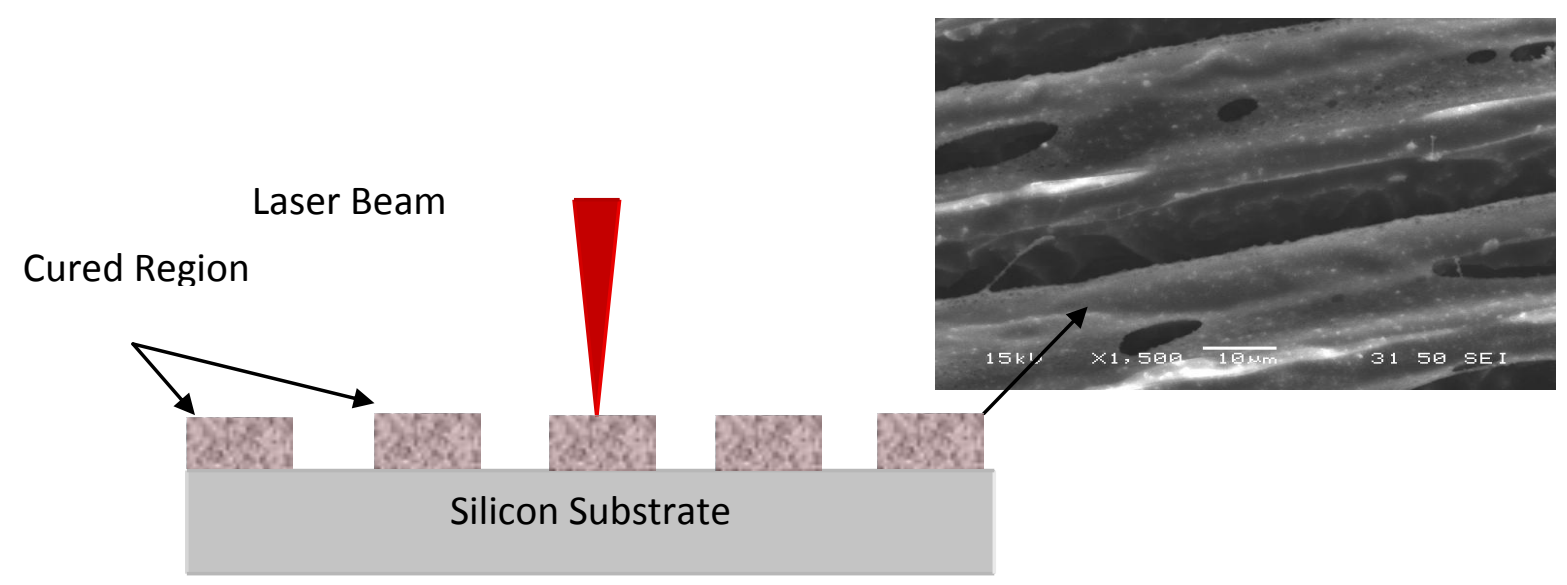

Fig 5.3 Irradiation of substrate

In order to investigate the formation of reinforced enclosed channels, series of experiments were conducted. It is noteworthy that enclosure of channels was achieved only with nanofiber fillings. When the experiment was repeated without nanofiber, no linking on the top occurred. Fig. 5.4a) shows a SEM image of a nanofiber reinforced self-enclosed channel and Fig. 5.4 b) shows a SEM image of generated microstructure without nanofiber by the proposed method. 

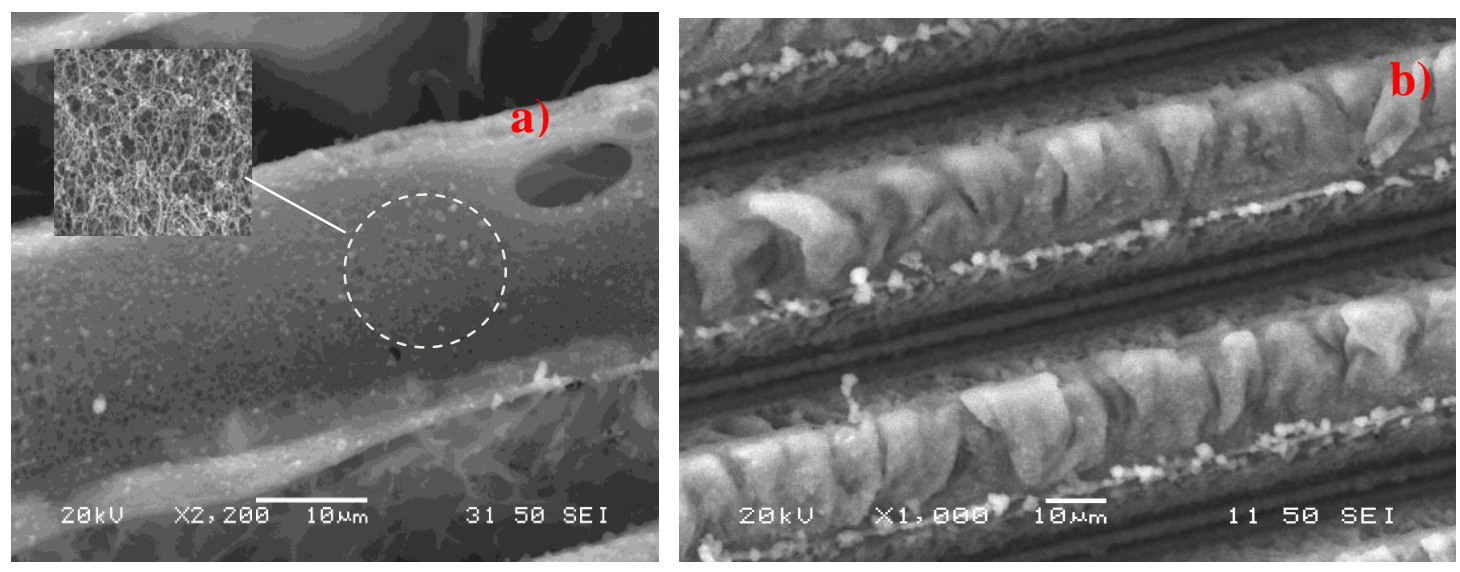

Fig 5.4 SEM Image of final microstructure created using this new process. a) SEM Image of final nanofiber reinforced self enclosed channel with a repetition rate of $13 \mathrm{MHz}$, an average power of $0.27 \mathrm{~W}$, a pulsewidth of $1428 \mathrm{fs}$, and a scanning speed of $1000 \mathrm{~mm} / \mathrm{s}$. b) SEM Image of microstructure created without nanofiber

Fig. 5.5 illustrates the linking mechanism by secondary curing. Upon laser irradiation, PDMS is solidified by the rapid heating. Above the solidified volume, there is a volume that is secondarily polymerized. It is not completely solidified, but practically cross-linked due to the residual heat of the laser beam. If a second scan pass is applied on the original scanned line, a complete cross-link could be induced at much lower laser intensity. If the subsequent scan pass is applied close to the original scan pass, the secondary curing will overlap with the practically cured part from the first scan and cause a roof bridges over the two solidified walls.

With pure polymers resin, the two sequent scan pass must be placed very close, less than $100 \mathrm{~nm}$, in order to induce a secondary curing. Previous study showed that secondary curing is possible without nanofiber fillings for photosensitive resin, such as SU-8 [116]. Whereas, in this experiment secondary curing did not happen for PDMS (which is thermosensitive) resin without any fillers (nanofibers). In presence of nanofiber fillings, 
there heat conduction along the fiber, making secondary curing possible with much wider spacing $(10-20 \mu \mathrm{m})$. Previous study shows that the nanofiber improves the heat conduction [117]. CROSSLINKING ON THE TOP
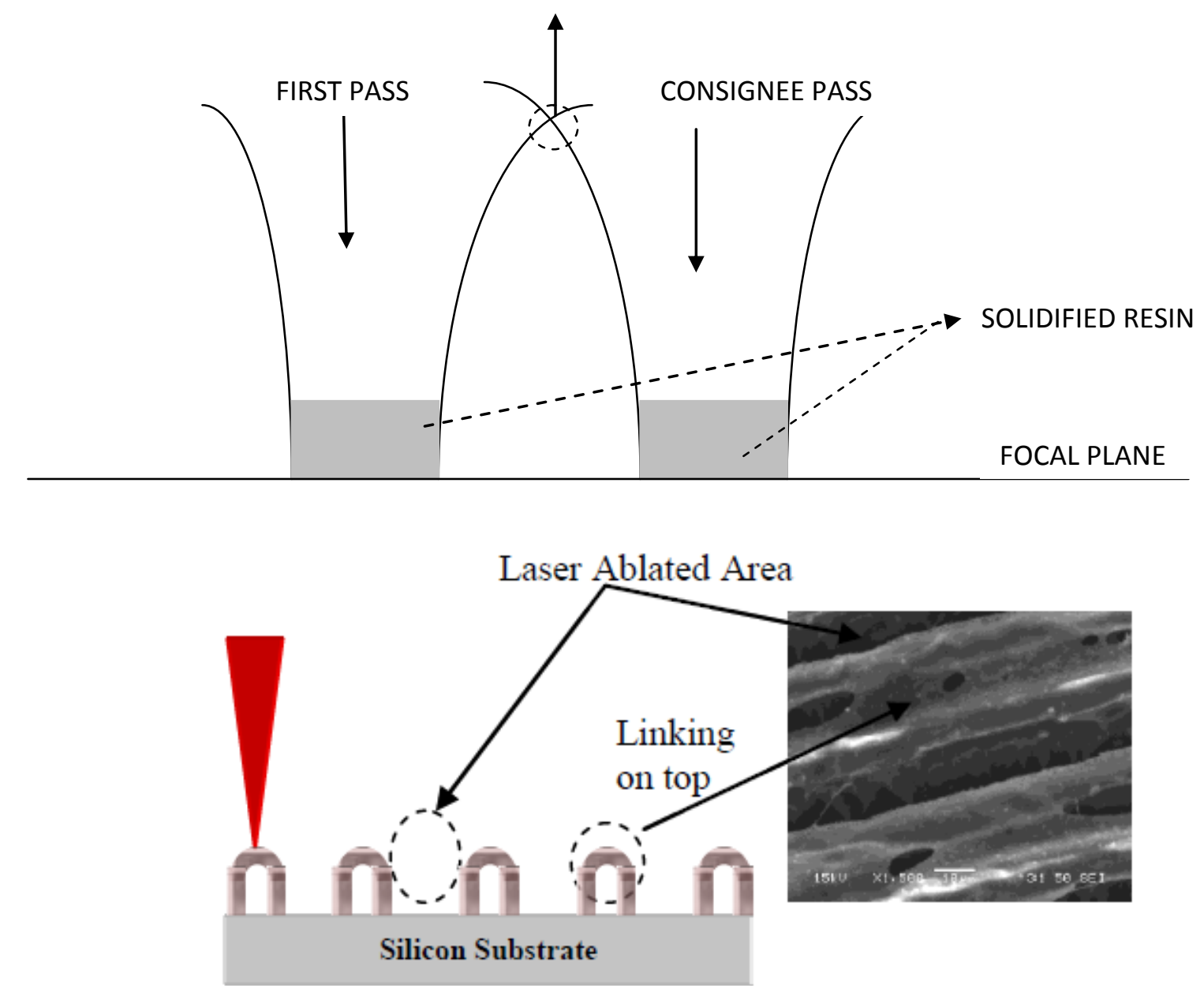

Fig. 5.5 Linking mechanism

Energy dispersive X-ray analysis was conducted on the sample, to analyze the composition of the generated micro features. Nanofiber reinforced PDMS and Pure PDMS were analyzed. The result showed in Fig. 5.6 confirmed the presence of silicon nanofibers in the roof of the reinforced polymer matrix. Comparing both pure and reinforced polymer, 
pure polymer contains low level of oxygen(34\%) and high level of silicon(65\%), where as the nanofiber-reinforced features contains a higher level of oxygen (43\%) and a lower level of silicon (57\%), which indicates of presence of silicon and silicon oxide nanofibers in the polymer matrix.

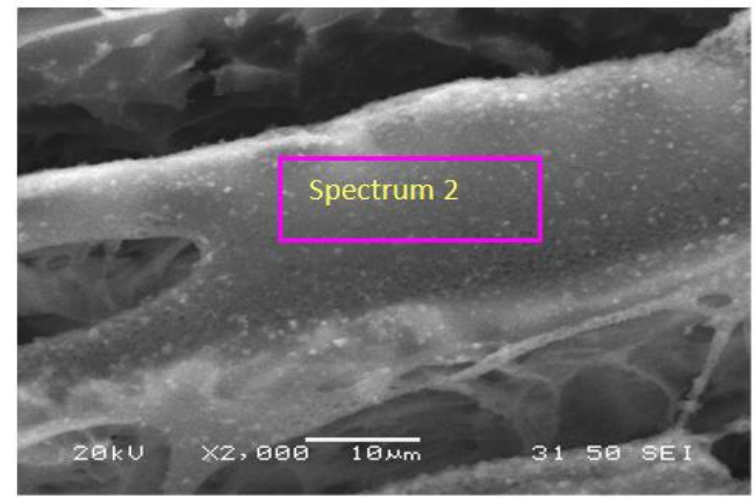

a)

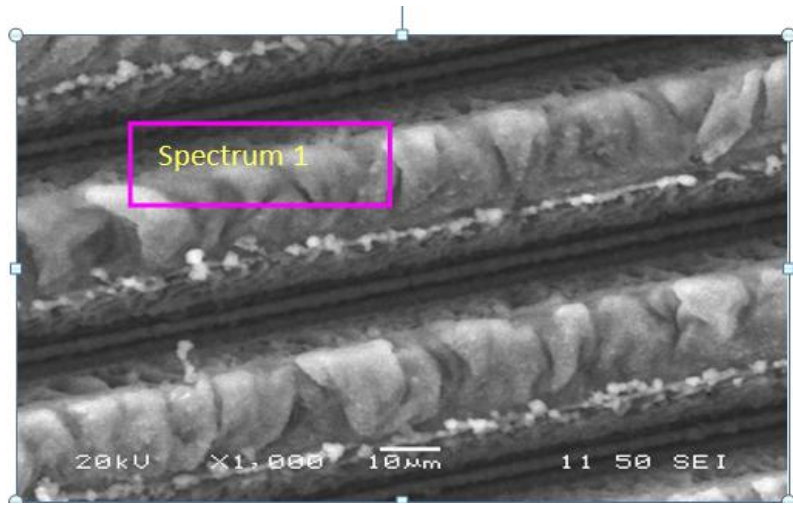

b)

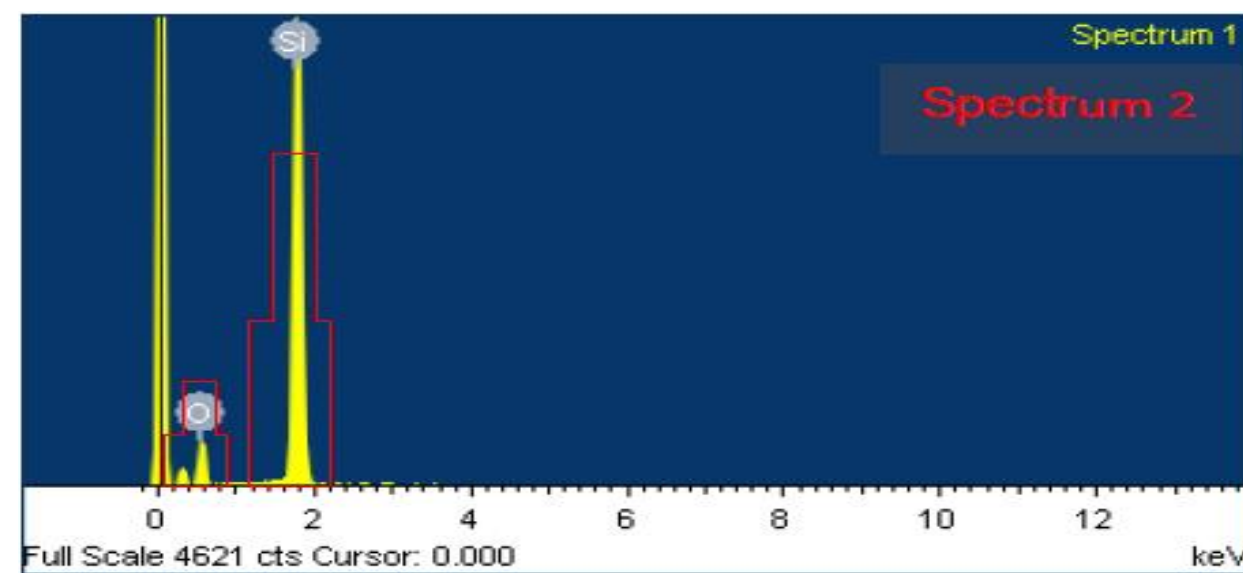

(c)

Fig 5.6 EDX analysis of a) Reinforced PDMS microstructures (Spectrum 2) and b) pure PDMS microstructure (Spectrum 1) (c) EDX spectrum

To investigate pulsewidth effect on the linking mechanism, laser pulses of duration of $214 \mathrm{fs}, 714 \mathrm{fs}$ and 1.428 ps were employed.. Fig. 5.7 shows a SEM image of a reinforced 
self enclosed channel created by the proposed method with a repetition rate of $13 \mathrm{MHz}$, an average power of $0.17 \mathrm{~W}$, scanning speed of $1000 \mathrm{~mm} / \mathrm{s}$ for different pulsewidth. The SEM images show that at a constant scanning speed the curing efficiency of PDMS improves with the increase of pulse width due to extended time of irradiation. As the pulsewidth increases, its diffusion increases due to the energy distribution. Enhanced channel formation is achieved at $1.428 \mathrm{ps}$ with $13 \mathrm{MHz}$ and high pulse energy.
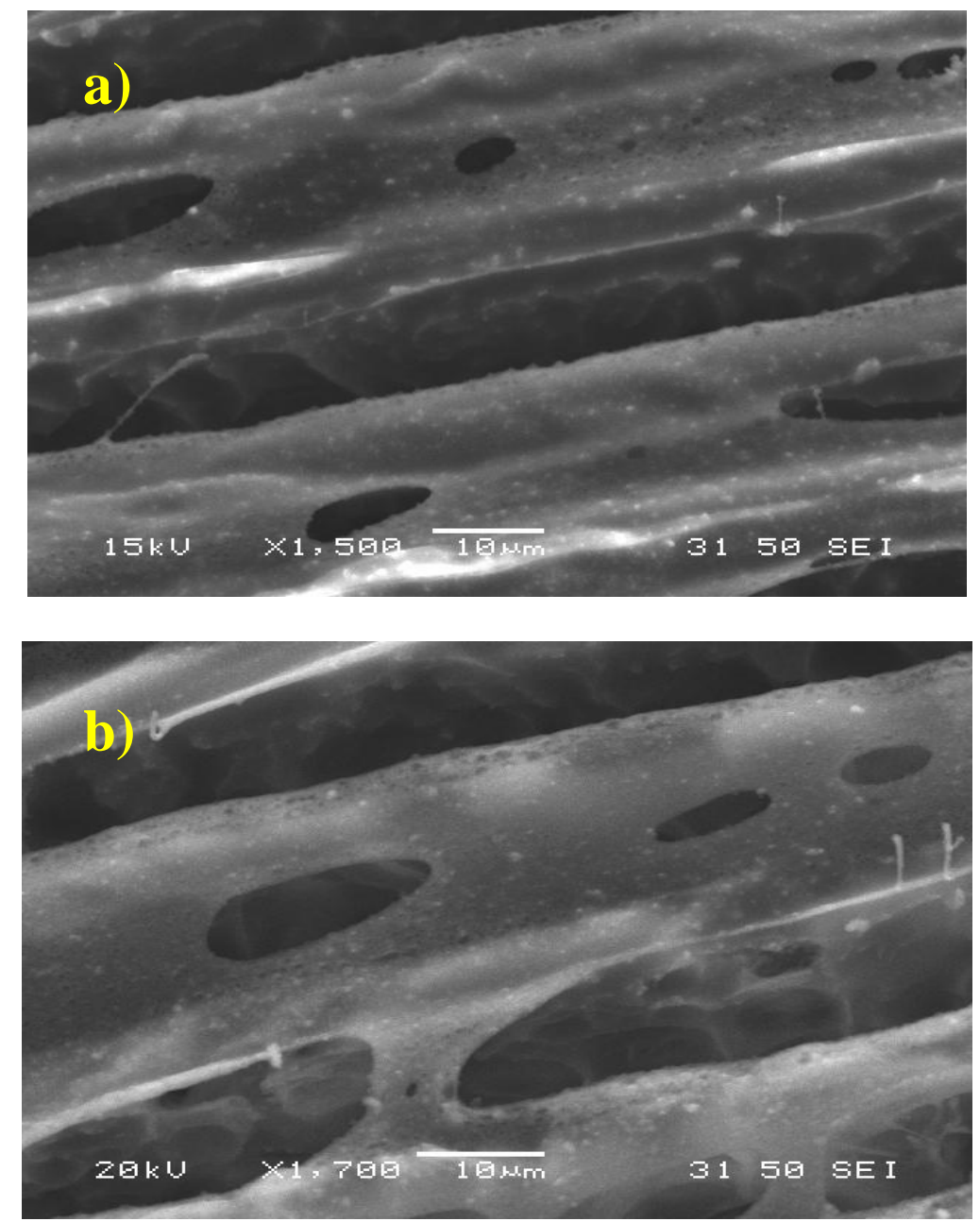


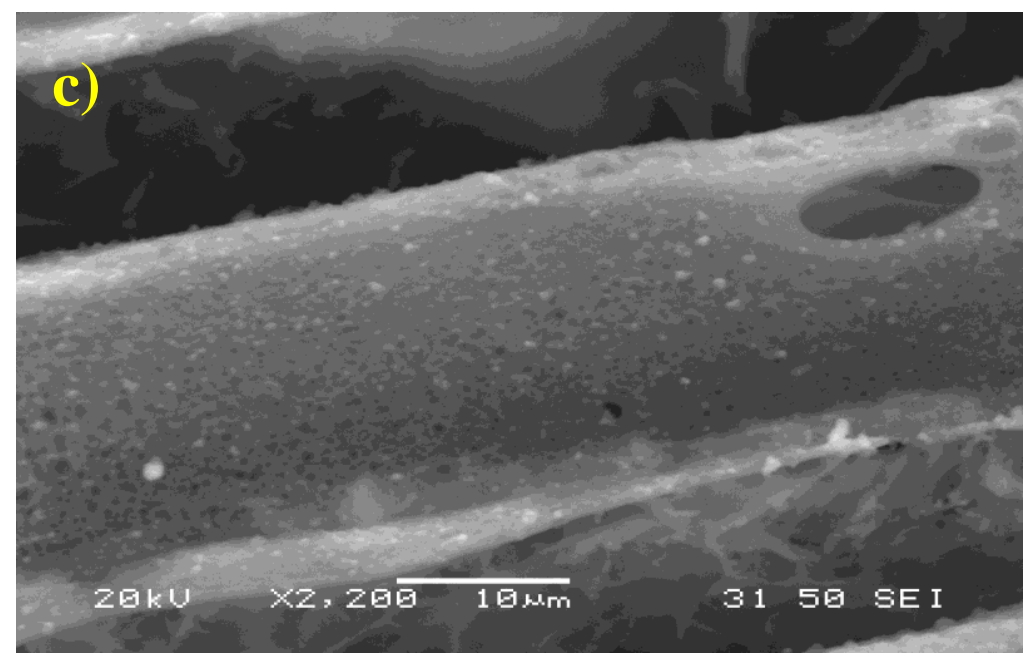

Fig 5.7 SEM image of reinforced self enclosed channels a) 714 fs- $13 \mathrm{MHz}-200 \mathrm{~mW}$, b) $1428 \mathrm{fs}-13 \mathrm{MHz}-250 \mathrm{~mW} \& \mathrm{c}) 1428 \mathrm{fs}-13 \mathrm{MHz}-270 \mathrm{~mW}$ (ZOOM Image)

\section{4.4 Test for Mechanical Properties of reinforced polymer}

A Nanoindentation test system was used to determine the strength of the reinforced polymer. In an instrumented indentation test, a diamond indenter tip (Berkovich tip, a threesided pyramid probe with a total included angle of $142.3^{\circ}$ and $5 \mu \mathrm{m}$ in radius), normal to the sample surface, is driven into the sample by applying an increasing load up to some preset value $(\max$ load $10,000.00 \mu \mathrm{N})$. The load is then gradually released until partial or complete relaxation of the material occurs. During both the loading (loading rate 20,000.00 $\mu \mathrm{N} / \mathrm{min}$ ) and unloading cycles (unloading rate $20,000.00 \mu \mathrm{N} / \mathrm{min}$ ), the applied force and penetration depth into the material are recorded (Fig. 5.8b), and a load-displacement curve (Fig. 5.8a) is created. The features of this curve (like slope) and testing parameters (such as contact area of the tip) allow material characteristics such as indentation hardness and Young's modulus to be determined. Figure 5.9 shows optical images of the indented sample. Nanoindentation analysis is done using the Oliver-Pharr method. Oliver-Pharr 
found that unloading data are usually not linear but are better described by a power law. The modulus from unloading is then calculated using the following equations:

$$
\begin{aligned}
& S=\frac{d p}{d H}=\frac{2}{\sqrt{\pi}} E_{r} \sqrt{A \beta} \\
& E_{r}=\frac{\left(1-v^{2}\right)}{E}+\frac{\left(1-v_{i}^{2}\right)}{E}
\end{aligned}
$$

where $\mathrm{S}$ is the measured stiffness of the upper portion of the unloading curve; $\mathrm{A}$ is the projected contact area; $E_{r}$ is the reduced modulus; $\beta$ is a correction factor which is 1.034 for a Berkovich indenter; E and $v$ are the Young's modulus and Poisson's ratio for the specimen, respectively; and $E_{i}$ and $v_{\mathrm{i}}$ are the Young's modulus and Poisson's ratio for the indenter, respectively.

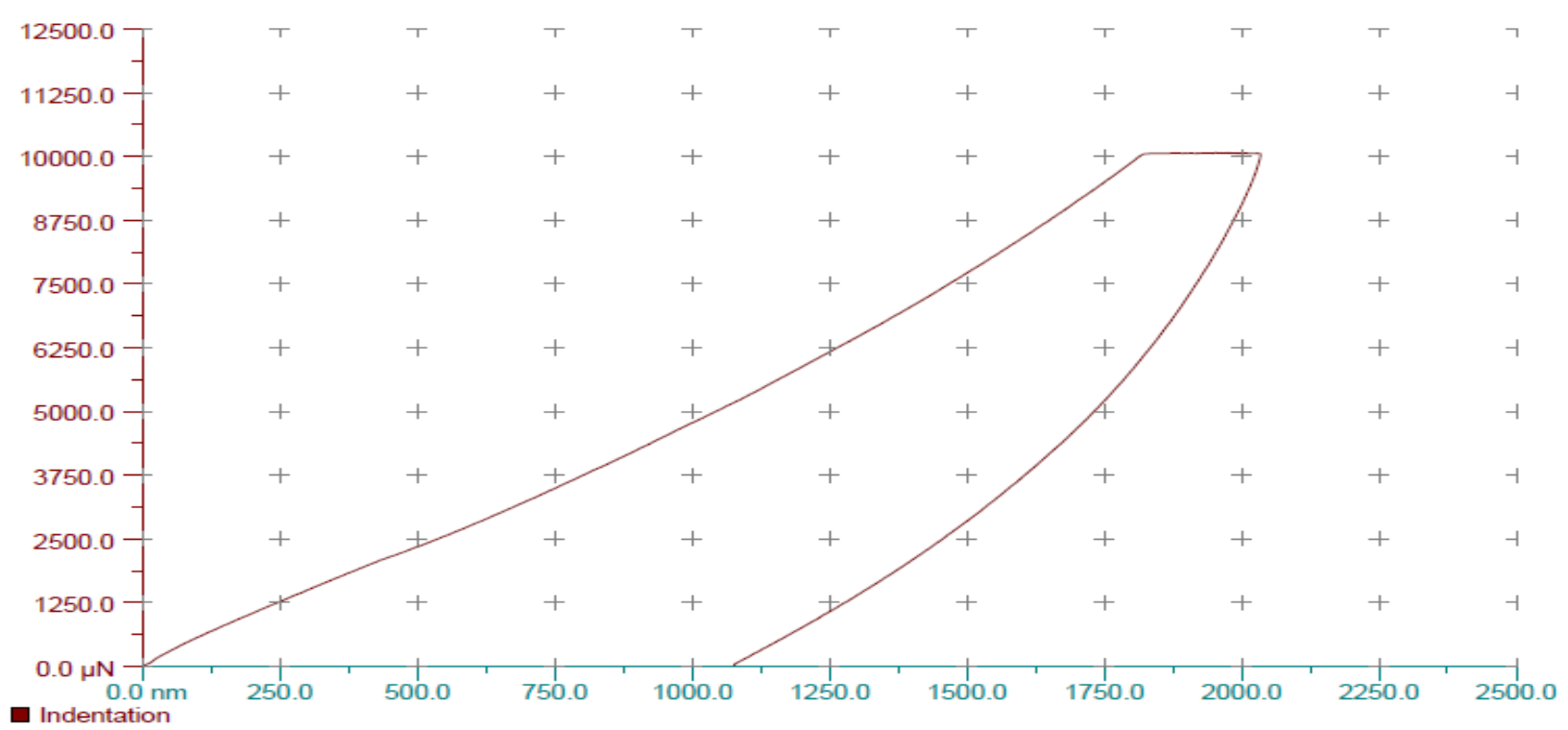

Fig 5.8 a) Load-displacement curve 


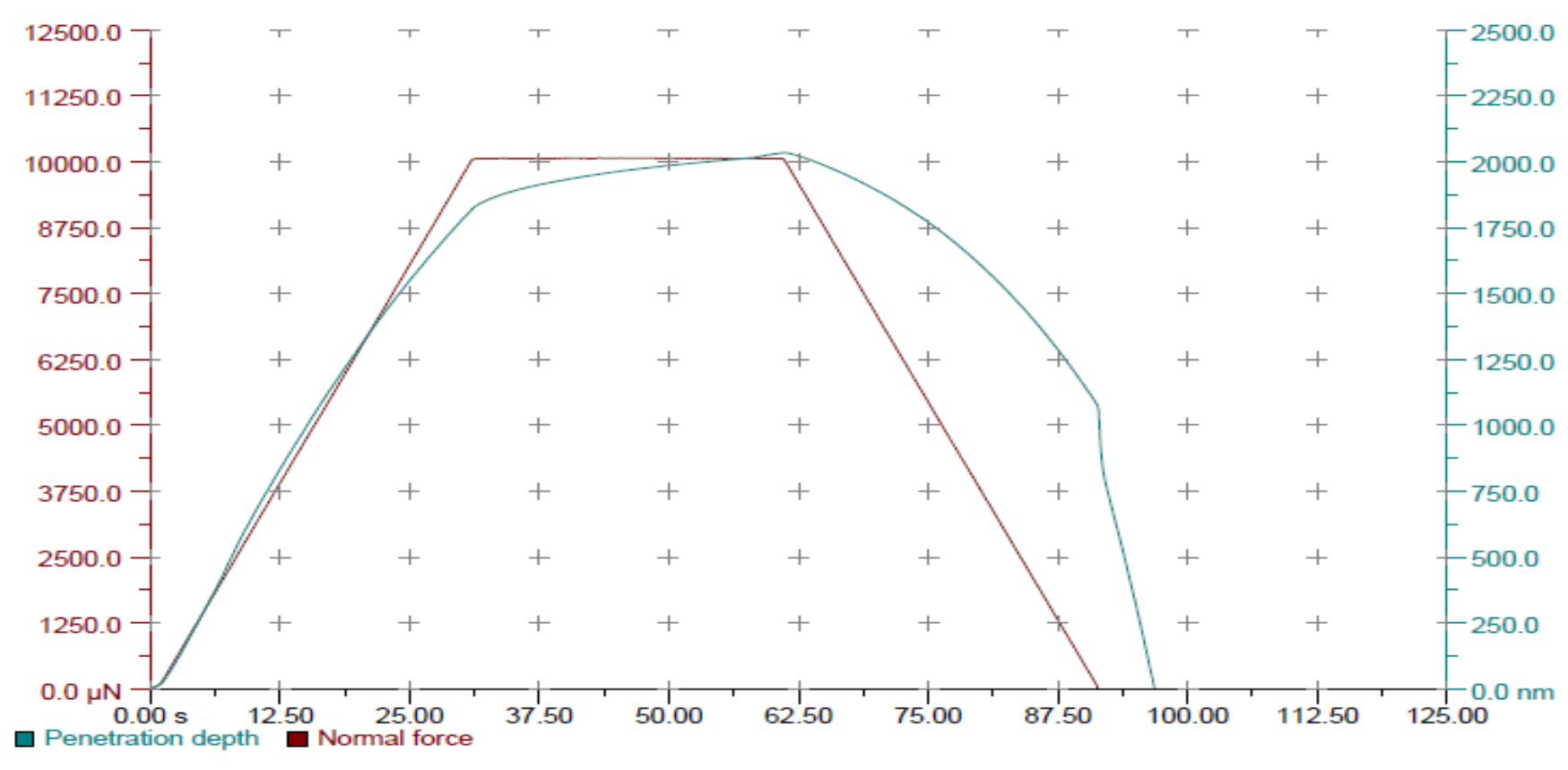

Fig $5.8 \mathrm{~b}$ ) Curve for the applied force and penetration depths

The indenter contact area can be calculated using the following equations. The area function for a perfect Berkovich indenter is the following.

$$
\begin{aligned}
\mathrm{A}\left(\mathrm{h}_{\mathrm{c}}\right) & =24.5 \mathrm{~h}_{\mathrm{c}}{ }^{2} \\
\mathrm{~h}_{\mathrm{c}} & =\mathrm{h}_{\max }-\mathrm{e}\left[\mathrm{P}_{\max } / \mathrm{S}\right]
\end{aligned}
$$

Where $\mathrm{P}_{\max }$ is the peak indentation load, e is 0.75 for a Berkovich indenter, $\mathrm{S}$ is the unloading stiffness, and $\mathrm{h}_{\max }$ is the maximum depth of indentation. 

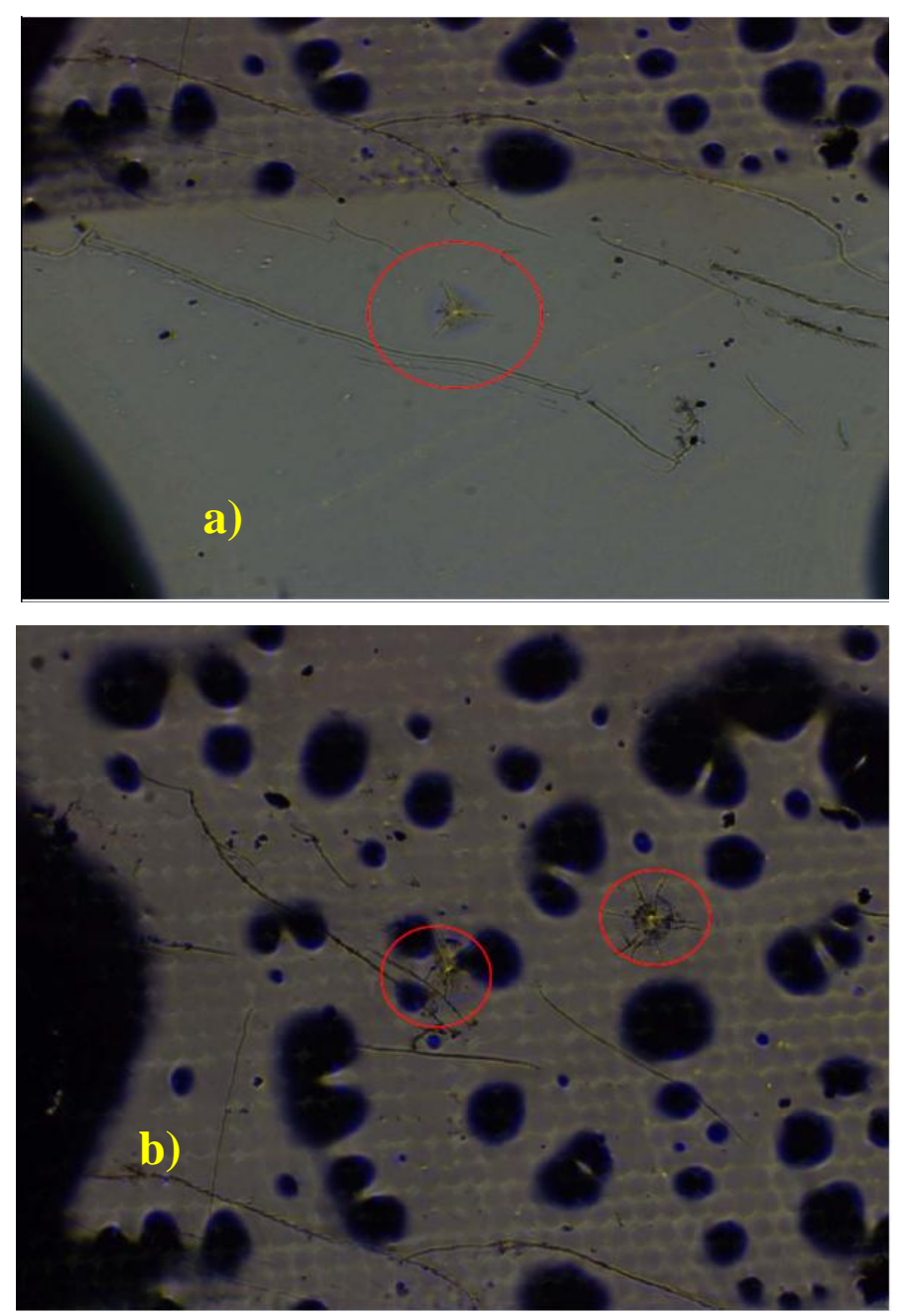

Fig 5.9 Optical Microscopy Image a) PDMS without Fibers b) With Fibers

The elastic modulus of pure and reinforced PDMS was calculated from nanoindentation analysis using the above-mentioned equations and it is $1.797 \mathrm{GPa}$ for PDMS with silicon nanofibers, and $0.175 \mathrm{GPa}$ for a pure PDMS sample. Clearly, incorporation of silicon nanofibers in to PDMS has a significant effect on mechanical property enhancement. The elastic modulus of PDMS increased up to 10 times with the addition of silicon nanofibers. 


\section{5 Summary}

A new method to develop polymer nanocomposites is reported. Ultrafast laser irradiation was used to generate nanofibers and cure nanofiber-filled polymer under ambient conditions. No additives were used in developing the nanocomposites. Selfenclosed channels were fabricated with the subsequent scanning lines are places close to each other (line spacing in the range of 10-20 $\mu \mathrm{m}$ ).

This process offers many advantages: decreased processing time; it is a non lithographic process; involves a straightforward fabrication process; and avoids misalignments due to thermal expansion or mismatch between the mold and the substrate, which is a problem for multilevel electronic device fabrication. Also, structures can be fabricated under non-clean room conditions and on any hard substrate irrespective of the materials (e.g., glass or silicon). The results pave the way for the new methodology of short pulse irradiation to be applied in the production of reinforced polymeric devices. To the best of the author's knowledge, this is the first time a reinforcement of PDMS induced by direct laser irradiation is reported. 


\section{CHAPTER 6}

\section{IRRADIATION AT HIGH LASER FLUENCE: SYNTHESIS OF PDMS-Si/AI HYBRID FIBROUS NANOSTRUCTURE}

When laser fluence is in the low to intermediate range, femtosecond laser irradiation is a useful tool for the fabrication of microstructures through rapid curing and ablation. If laser fluence is high above the ablation threshold of PDMS, an entirely different phenomenon occurs and yields PDMS nanoparticles and PDMS-Si/Al hybrid nanostructure.

\subsection{Introduction}

Much research has been reported on Nanocomposites, but far few studies have been conducted on hybrid nanostructures. Nanocomposites are generally described as a bulk matrix reinforced with nanoscale materials. Hybrid nanostructures also involve the combination of two or more constituents. What makes hybrid nanostructure different from nanocomposite is that in hybrid nanostructures all constituents are in nanoscale. Due to this characteristic, hybrid nanostructures may raise potential synergistic properties, thus potential applications in various areas. Current methods of synthesizing polymer/inorganic hybrid nanostructures is limited to wet - chemical techniques, including molecular beam epitaxy, chemical vapor deposition, Sol-Gel process , In-situ polymerization, , benzyl alcohol route[118], hydrothermal route[119-120], miniemulsion polymerization[121] and etc. With wet-chemical techniques, individual nanoparticles of two or more different materials are mixed and linked into two dimensional structures, such as chains, through 
chemical reactions. A variety of hybrid nanostructures have been synthesized and demonstrated for diverse applications: lithium ion batteries, light harvesting, gas sensors, nanobiosensors, energy storage, cellular imaging, biomedical and photo thermal imaging, data storage, photovoltaics and therapeutic [122-124]. B. P. Vinayan et al. [125] synthesized graphene-multiwalled CNT hybrid nanostructure for lithium ion battery applications. For this, graphene was prepared by the solar exfoliation of graphite oxide, while MWCNTs are prepared by chemical vapour deposition method. The graphene and MWNT are chemically modified before generating graphene-MWNT hybrid nanostructure. Hybrid nanostructure is attained by homogeneous mixing of chemically modified graphene and MWCNT. S.Mackowski [126] demonstrated a method to design novel hybrid nanostructure comprising biological photosynthetic systems (natural photosynthetic protein-pigment complexes) and nanostructures which includes semiconductor nanocrystals, metallic nanoparticles or carbon nanotubes. These hybrid structures enhances the efficiency of light harvesting either via Plasmon excitation in metals or absorption tunability characteristics of semiconductors. Such hybrid systems, through combining the properties of the constituents, provide a novel paradigm for designing photovoltaic devices. Shaoli Zhu et al. [127] reviewed the design, fabrication and applications of hybrid Au-Ag triangular nanostructures. The hybrid nanostructure (Au-Ag triangular nanostructure) is prepared using Nanosphere Lithography technique. The refractive index sensitivity of hybrid Au-Ag triangular nanostructures is calculated by FDTD method, and the sensitivity in the experiment is detected using protein. This hybrid $\mathrm{Au}-\mathrm{Ag}$ nanostructure is used to detect SEB solution. The detection sensitivity for SEB is nanogramme per milliliter level. 
The experiment results show that the hybrid nanostructures are useful in the nanobiosensors research field, which has many potential applications in chemical material research fields. The prevailing techniques to generate hybrid nanostructures have various drawbacks: generates nanoparticulate structures (1D and 2D structures) on certain composition of materials, difficulties in control of structure and morphology, excessive use of solvents as medium which may leave toxic residues in the final product, abundant energy consumption, and multiple steps which requires long processing time.

Recent efforts studied the different methods for generating PDMS nanostructures (e.g., nanowell, nanopost, nanopillar arrays) namely RIE etching, Nanosphere Lithography and two step moulding process. In recent times, femtosecond laser have proven to be powerful means for nanostructuring of bulk metals. Femtosecond laser pulses have also been used for distinct nanostructuring of thin films with minimal thermal side effects [128130]. Laser ablation of polymeric material usually results in complete decomposition of the original polymer. The laser ablative synthesis of polymeric nanoparticles had never reported before.

\subsection{Hybrid Nanostructure Generation}

First, nanofibrous structures are formed by laser ablation of PDMS coated silicon target in ambient atmosphere. Arrays of holes were drilled in to the silicon substrate using

femtosecond laser of $13 \mathrm{MHz}$ pulse frequency with dwell time of $10 \mathrm{~ms}$ and a power of 15W. Previous experiments [73] revealed that the formation of fibrous nanostructure maximizes at 12.6 MHz. Thus, the experiments were conducted at a frequency of $12.6 \mathrm{MHz}$ in order to obtain the highest production. 
Hybrid PDMS-Si and PDMS-Al fibrous nanostructures are produced in a single step process, using femtosecond laser pulses of $\mathrm{MHz}$ pulse frequency involving laser ablation. This work deals with a new strategy that applies an ultrafast laser beam on a thermo sensitive resin to achieve laser ablation. Using SEM/TEM, it is observed that individual nanoparticles are bonded with each other to form nanoparticle aggregate

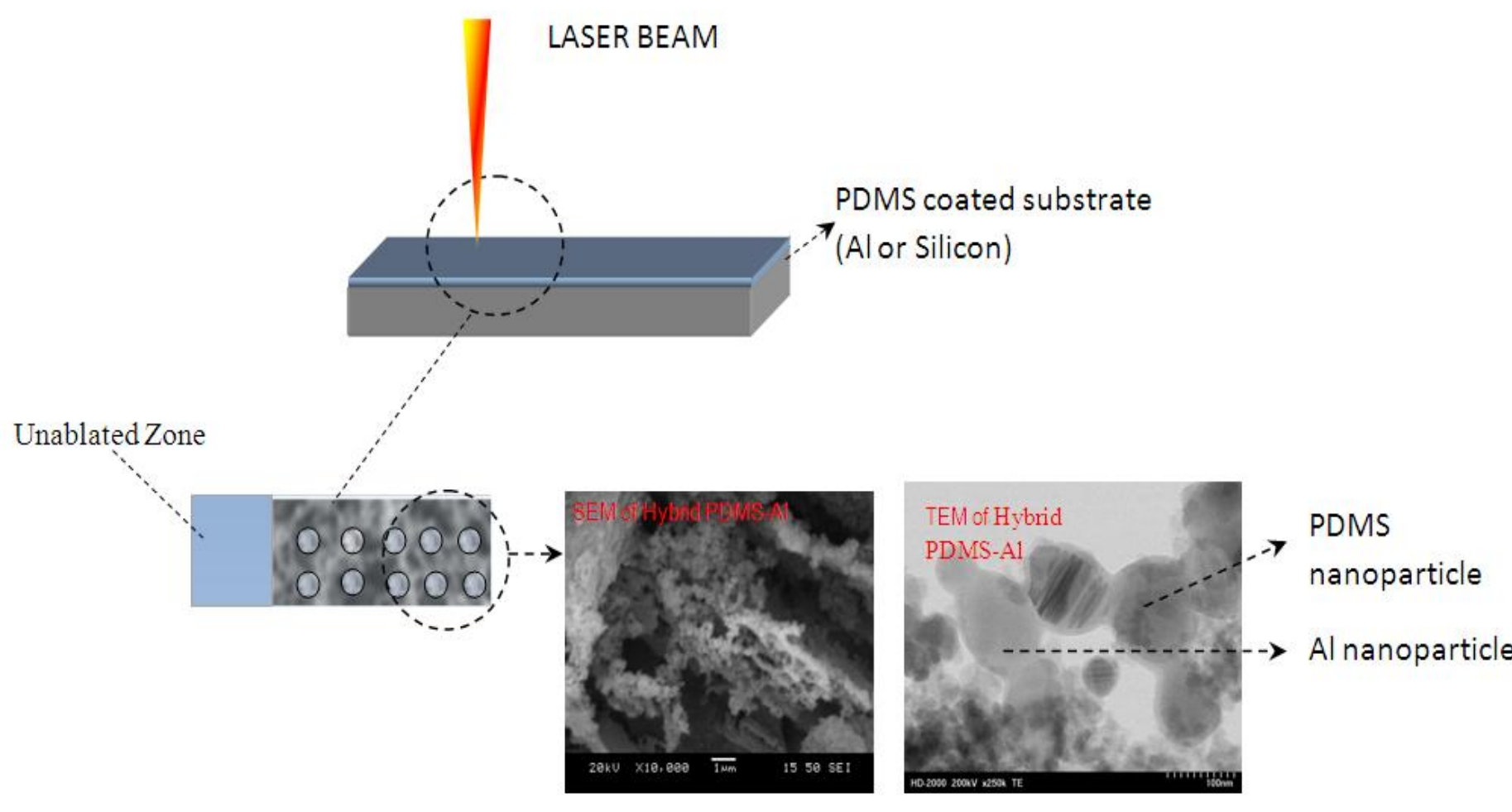

Fig 6.1 Proposed single step direct synthesis of hybrid nanostructure 


\subsubsection{PDMS-Al Hybrid Nanostructure}

To explain the process of hybrid PDMS-Al nanofibrous structure generation, the experiments are categorized in to three regimes based on the coating thickness. The process that explains the PDMS nanocomposite generation with aluminum fibers begins with laser pulses striking the substrate at the interface aluminum- PDMS) to induce plasma and commence the ablation process that gradually alters the surface morphology. Irrespective of the substrate (silicon / aluminum), only polymer is ablated in the first regime. At the polymer - substrate interface (about 20 -23 microns) both polymer and substrate are ablated. At depths greater than 23 microns only the substrate is ablated. These numbers are an approximation based on the coating thickness of the polymer. The hybrid structure formation is explained using liquid vapor condensation.

Vapor condensation in the plume of laser ablation generates nanoparticles and they aggregate [73]. This method demonstrates a unique, novel and inexpensive way of creating 3D fibrous nanostructure. Vapor condensation starts with nucleation, proceeds with growth of supercritical nucleus and comes to a halt due to the effect of quenching. The observed structure is an aggregate of particles of few tens of nanometers. This indicates that nanoparticle aggregation occurs in the latter part of the vapor condensation by the collision of large sized nucleus. The density of nucleus decreases as the plumes expands. For nanoparticles to aggregate in massive scale and form fibrous nanostructures, a continuous source of vapor needs to be supplied to the expanding plume in order to maintain the nucleus density at a certain level. The plumes generated by consecutive laser pulses must arrive before the critical time. It is also found that the pulse frequency at which the particle 
aggregations form is in agreement with the theoretically calculated critical time to start nanoparticle formation [115]. The fibrous nanostructure formed shows certain degree of self-assembly consisting of rings and bridges. Also, the fibrous nanostructures have relatively uniform diameters $(20 \mathrm{~nm})$ and did not observe a wide range of variation in size distribution. The mechanism of the particle-aggregate formation can be explained by laser ablation and gas phase condensation. Fig 6.1 shows the proposed method to generate hybrid nanostructure.

Recently, several experiments have been performed on laser ablation of solid targets. Due to the very short time scales involved in the ablation with femtosecond laser pulses, the ablation process can be considered a direct solid-vapor (or solid-plasma) transition. But no research has been done on liquid vapour condensation of PDMS by laser ablation. In this case, the femtosecond laser is tightly focused on to the liquid resin (PDMS coated $\mathrm{Si} / \mathrm{Al}$ substrate), which leads to a direct phase transformation from liquid to vapour.

SEM micrographs of the surface of PDMS coated aluminum sample around the laser-irradiated spots are shown in Figure 6.2a. Three-dimensional interweaved nanofibrous structures are observed in and around the laser irradiated spots with relatively uniform diameter in the order of $20 \mathrm{~nm}(\mathrm{Fig} 6.2 \mathrm{~b}, 6.2 \mathrm{c})$. Transmission electron microscope(TEM) EDX line analyses run across a few particles of the fibrous nanostructure shown in Fig 6.2b \& 6.2c. It is evident from TEM- EDX analysis that there is no much oxidation of aluminum nanoparticles. Fig. 6.4 shows Raman spectra of treated and untreated PDMS coated Al sample. The peaks a, b, c and d are attributed towards PDMS in the untreated sample. The peaks e, $\mathrm{f}, \mathrm{g}$, and $\mathrm{h}$ are attributed towards PDMS in the treated sample. The observed peaks 
for PDMS in both treated and untreated sample, indicates the presence of PDMS in the treated PDMS-Al sample. The observed PDMS peaks also agreed fairly well with those available in the literature [131]. It indicates that PDMS didn't not undergo chemical reaction during laser treatment and remains intact in the final hybrid structure.
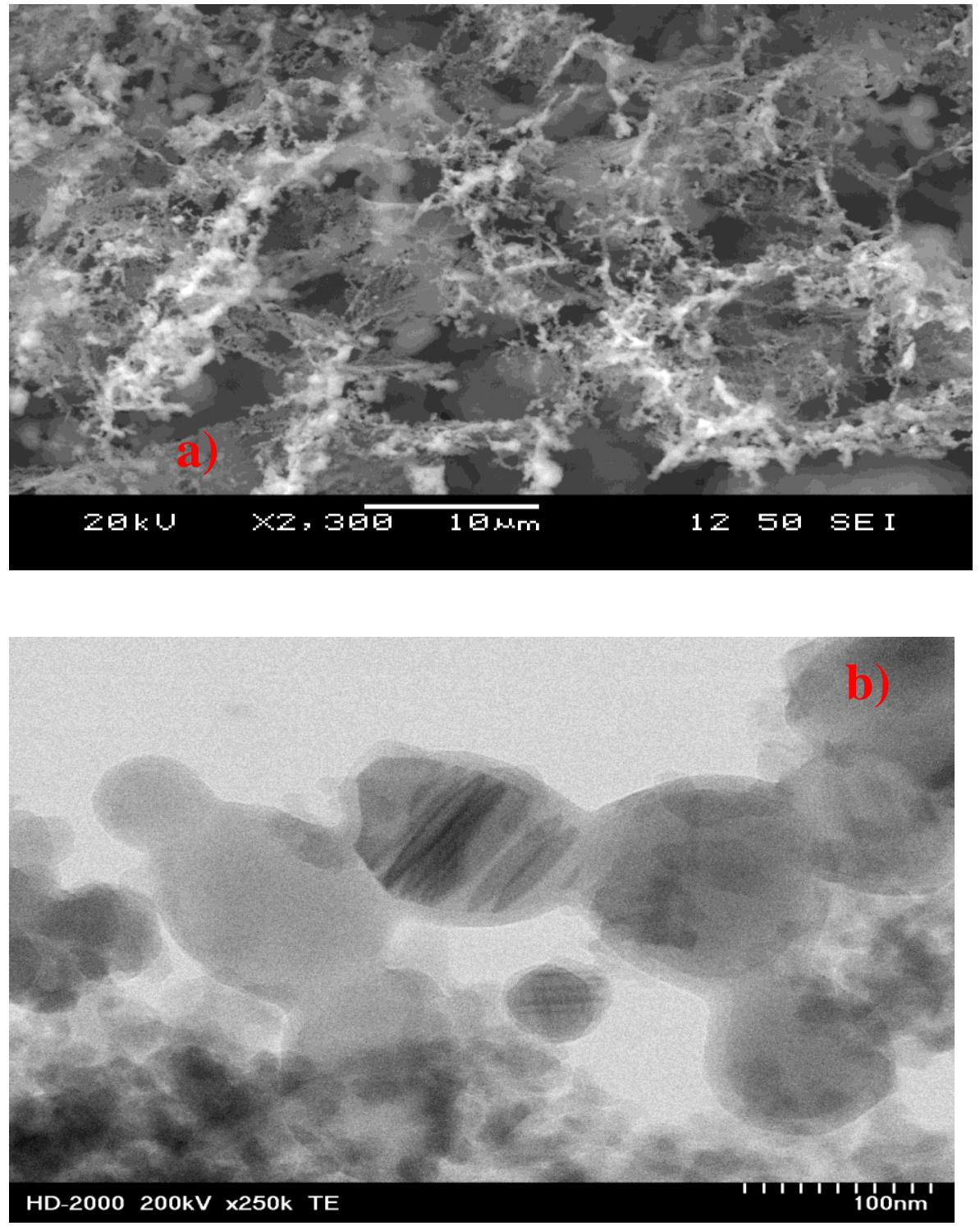


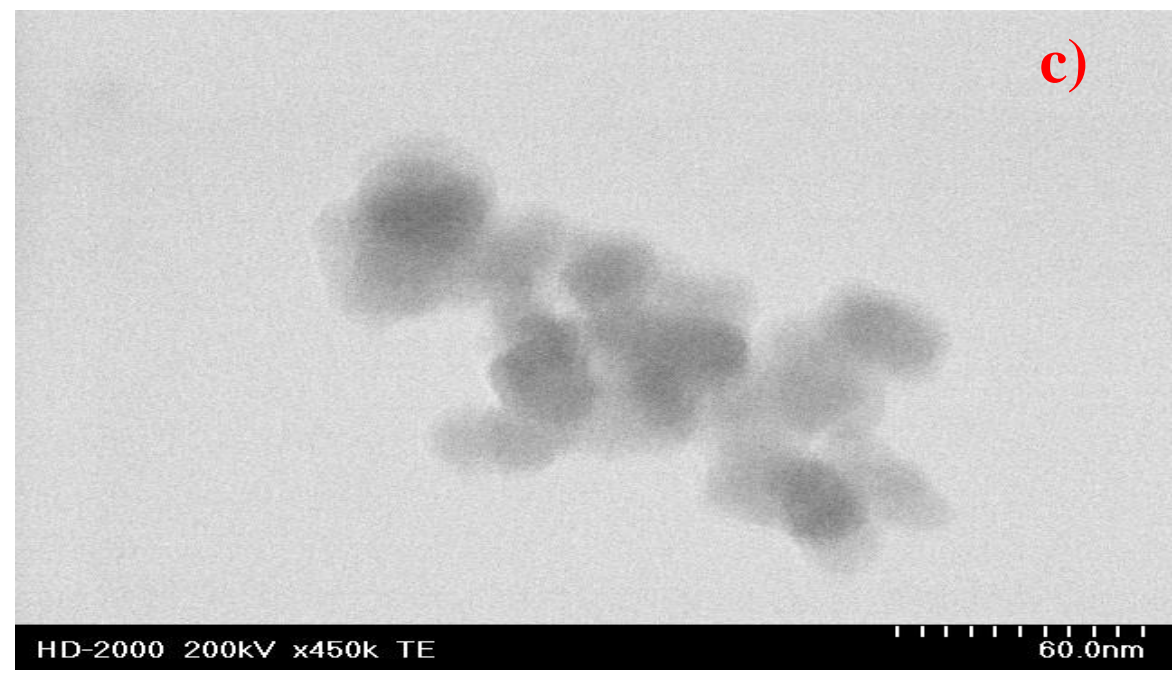

Fig 6.2 SEM and TEM micrographs of the laser irradiated PDMS coated aluminum sample surface and the nanoparticles aggregate respectively. (a) Surface featuring web like nanoparticles around the laser spot, (b, c) PDMS-Al /Al nanoparticles in the aggregate structure

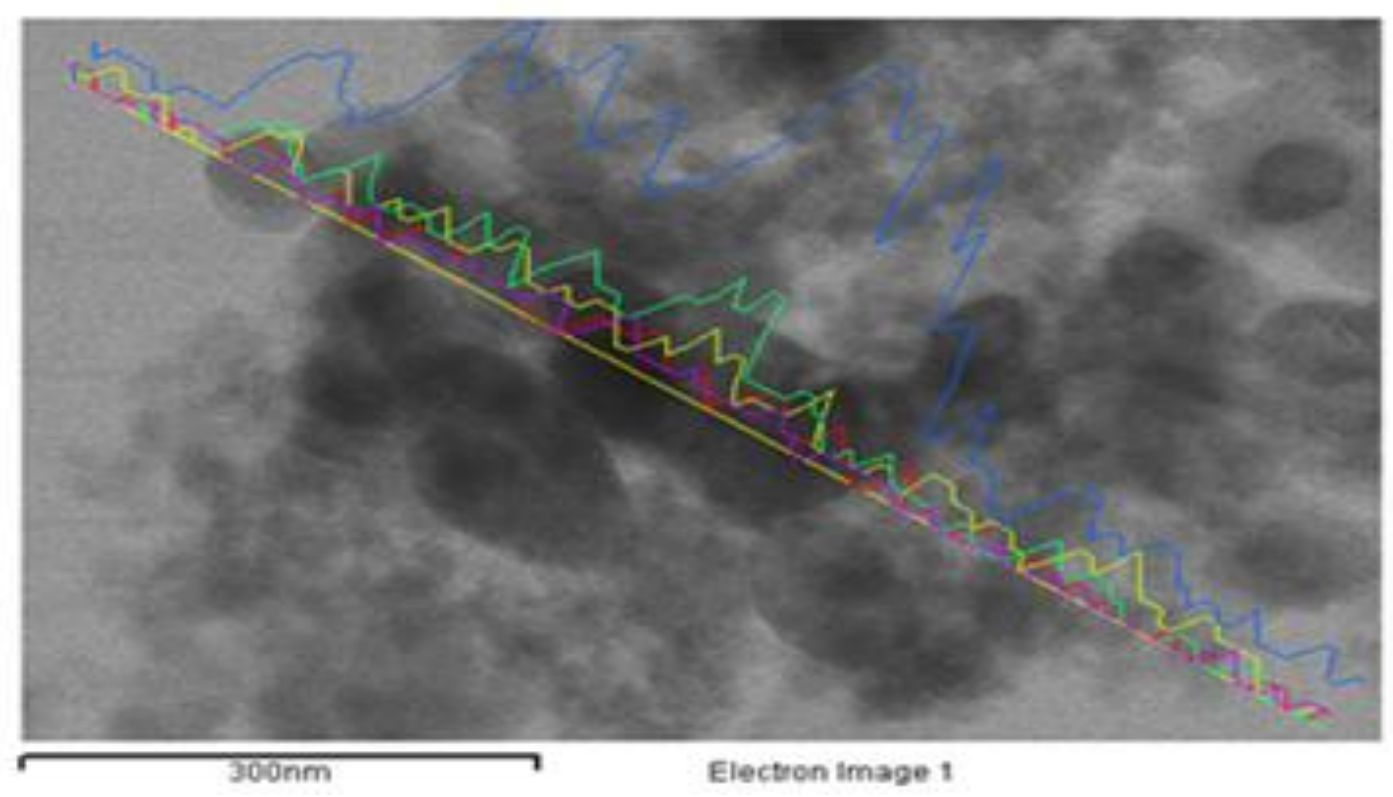



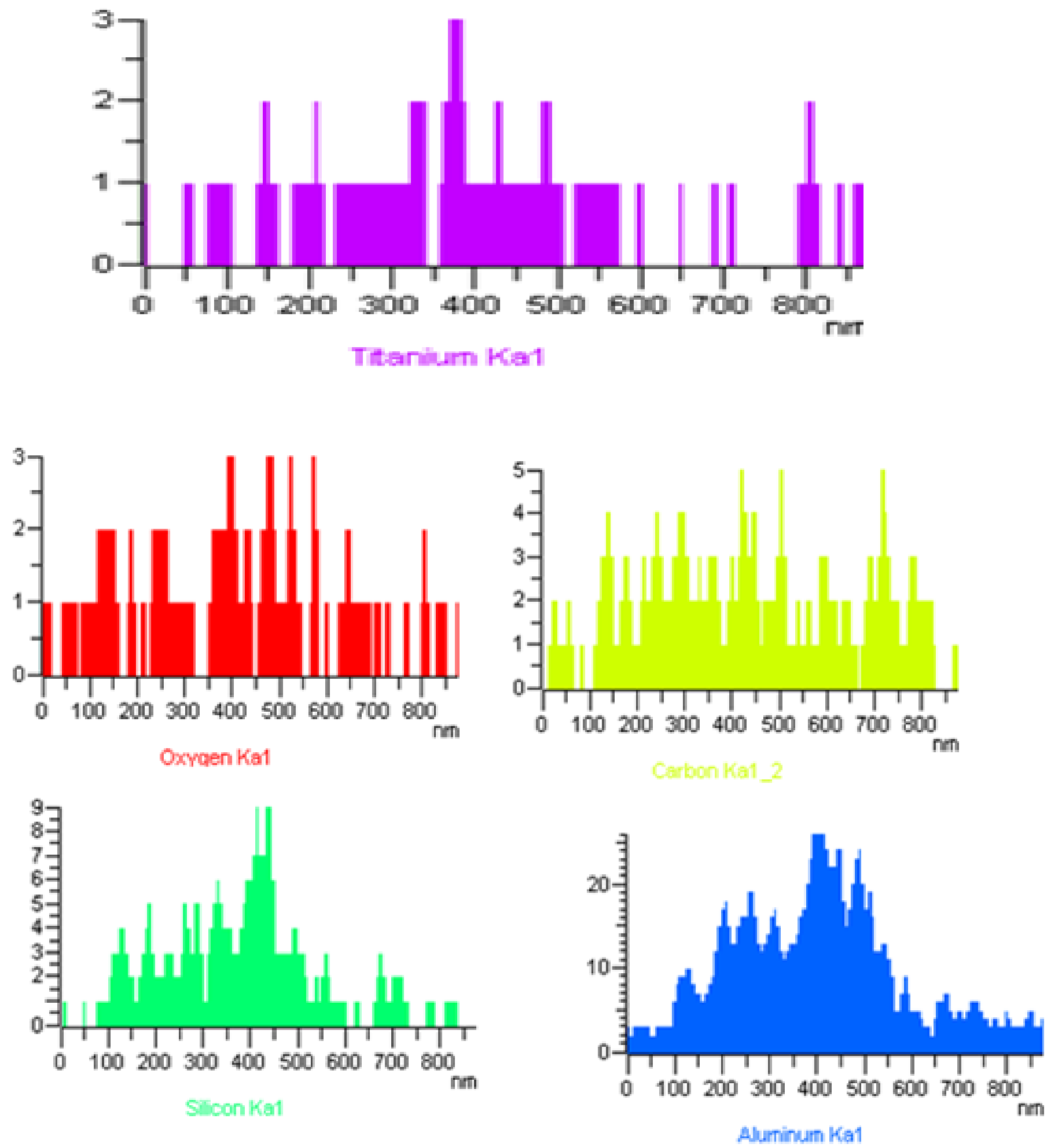

Fig 6.3 TEM-EDX analysis of the nanoparticles in the aggregate structure (PDMS coated aluminium substrate) 


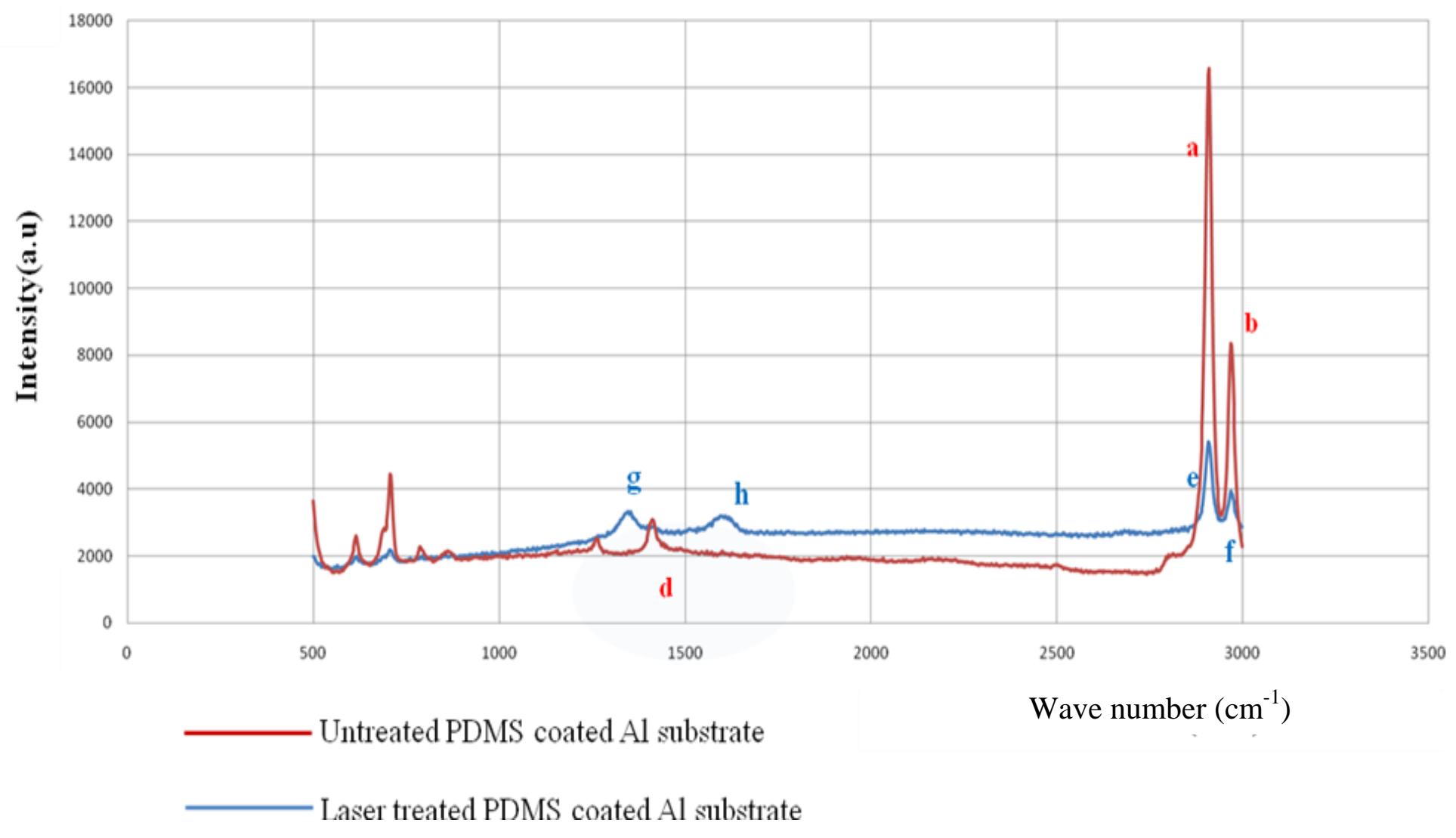

Fig 6.4 Raman Spectra for treated and untreated PDMS coated Al sample

TEM-EDX analysis (Fig 6.3) confirms the presence of aluminum in the hybrid structure. It is evident from TEM -EDX analysis; there is no much oxidation which indicates the existence of aluminum in the final hybrid structure. It indicates that $\mathrm{Al}$ nanoparticles are enclosed by PDMS shells before they oxidize inside the plume. This may confirm the formation of possible core-shell hybrid PDMS-Al fibrous nanostructure. Further TEM-EDX shows that the elements in the PDMS-Al hybrid nanostructure such as aluminum, carbon and silicon are evenly distributed, which shows that the vapor condensation is the most possible particle formation mechanism for both PDMS as well as 
the substrate material (aluminum). It could be observed from SEM /TEM images, that fibers were formed by the agglomeration of the spherical nanoparticles. Morphology and the size [73] of the nanoparticles is evidence for vapor condensation and nucleation as the particle's forming mechanism. Raman spectra and TEM-EDX analysis confirms the existence of aluminum and PDMS in the final hybrid nanostructure. Generally, upon laser irradiation on a thermosensitive resin, the laser pulses initiate the photochemical process and the absorbed optical energy is instantaneously converted into thermal energy, causing rapid temperature rise and a chemical reaction that induces polymer solidification. But, in this work probably polymer undergoes a direct phase transition from liquid to vapor. Then the vapour condensate and aggregate to enclose Al nanoparticles to form hybrid PDMS-Al nanostructure. The above observations could be used to explain the morphology of fibers generated on PDMS coated aluminum substrate.

\subsubsection{PDMS-Si Hybrid Nanostructure}

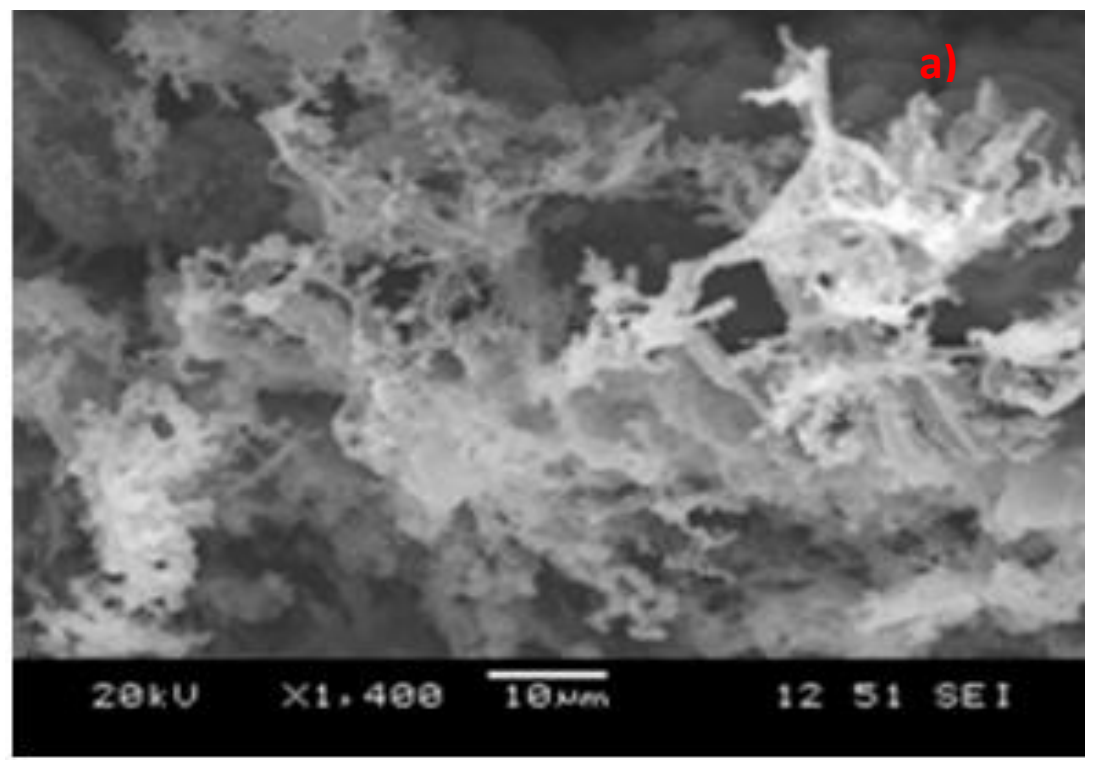



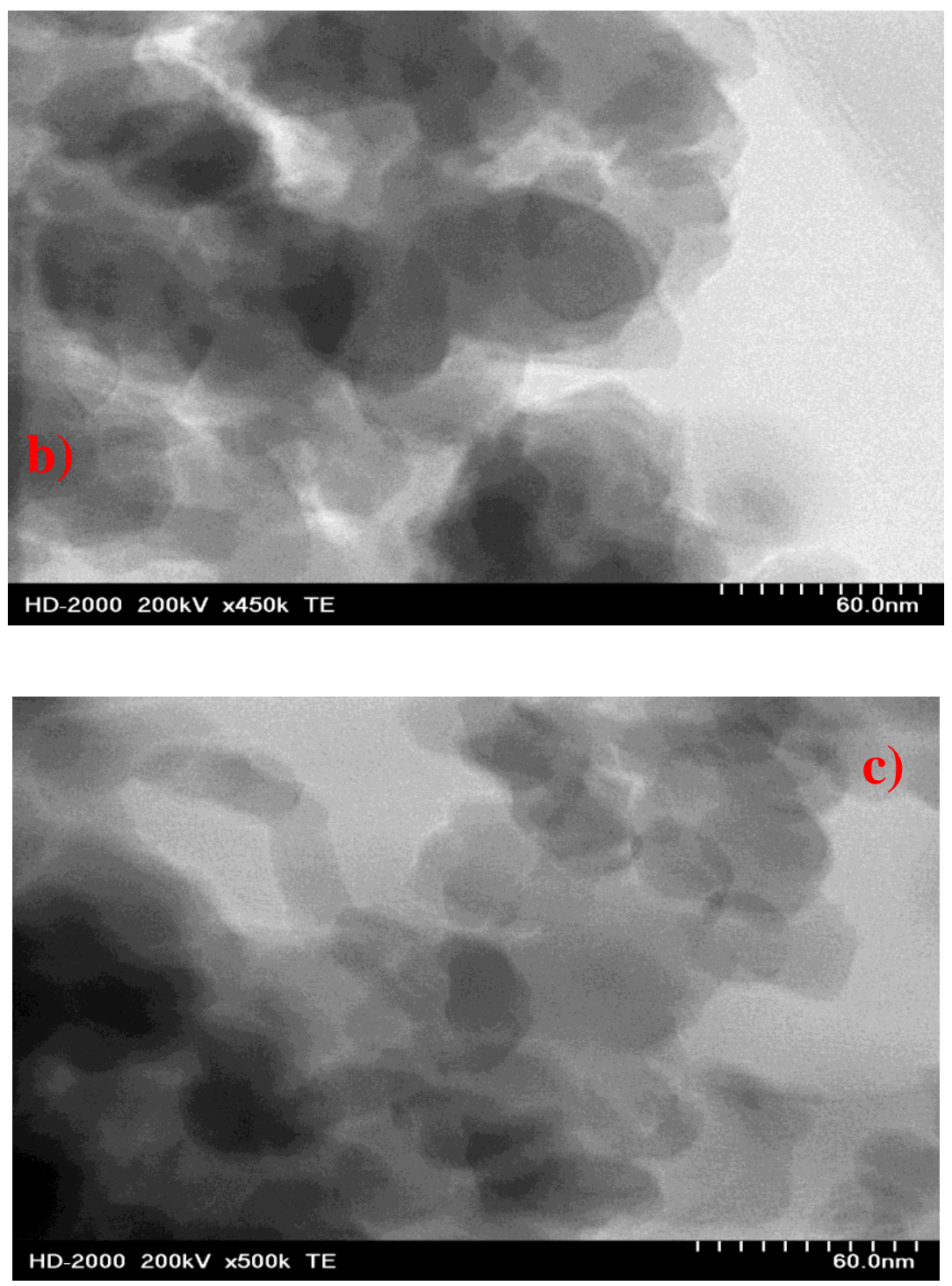

Fig 6.5 SEM and TEM micrographs of the laser irradiated PDMS coated silicon sample surface and the nanoparticles aggregate respectively. (a) Surface featuring web like nanoparticles around the laser spot, (b, c) PDM-Si / Si nanoparticles in the aggregate structure

In similar fashion, experiments were repeated with silicon substrate. Figure 6.5a shows the SEM micrographs of the surface of PDMS coated aluminum sample around the laser-irradiated spots. 

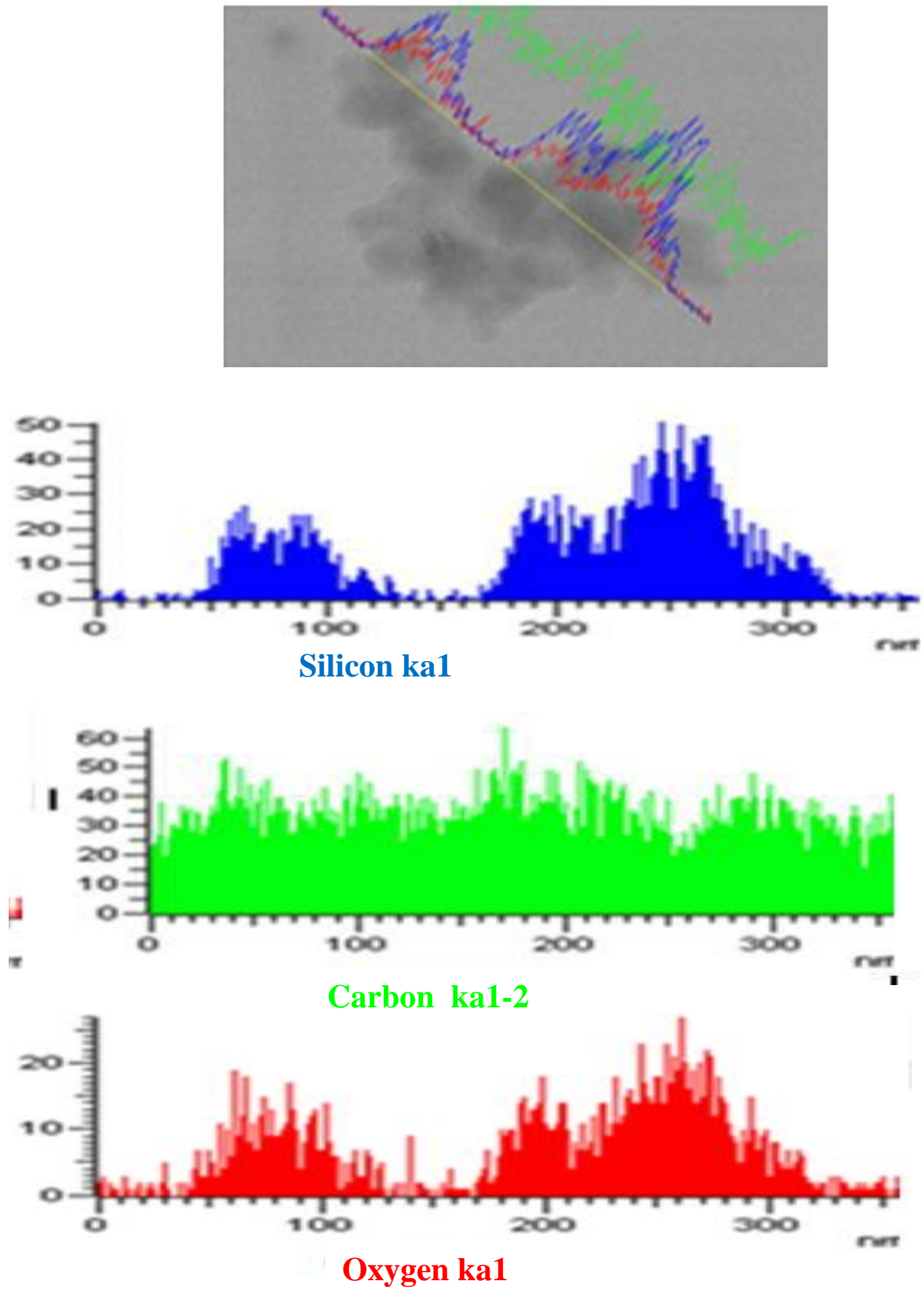

Fig 6.6 TEM-EDX analysis of the nanoparticles in the aggregate structure (PDMS coated Si substrate). 
It is evident from TEM-EDX, that the relative amount of PDMS, Si in PDMS-Si nanoparticle varies with different particles (Figure 6.6). Elements in the PDMS-Si hybrid nanostructure are evenly distributed. Even distribution shows that the vapor condensation is the possible particle formation mechanism for both PDMS as well as the silicon. TEMEDX ratio analysis reveals the existence of silicon in the final hybrid structure. It could be observed from SEM images, that fibers were formed by the agglomeration of the spherical nanoparticles. Morphology of nanoparticles is evidence for vapor condensation and nucleation as the particle's forming mechanism. The above observations could be used to explain the morphology of fibers generated on PDMS coated silicon substrate.

The proposed method offers several advantages: involves straightforward generation process; decreased processing time; does not involve any chemicals/additives; dry technique. Also hybrid nanofibrous can be generated under non clean room conditions and on any materials.

\subsection{Summary}

When irradiation laser intensity is high above the ablation threshold of PDMS resin, direct vaporization occurs and PDMS resin was converted into nanoparticles. Material characterization showed that the formed nanoparticles have the same chemical composition and characters as PDMS resin. To the best of the author's knowledge, this is the first time that polymeric nanoparticles were generated by laser ablative synthesis.

If PDMS resin and other solids, i.e. Aluminum and Silicon, are ablated simultaneously, hybrid PDMS-Si and hybrid PDMS-Al nanoparticle networks will be 
generated. The nanoparticles network is a fusion of PDMS nanoparticles and aluminum or silicon nanoparticles. A small amount of these hybrid nanostructures show a core-shell structure, with silicon or aluminum nanoparticles in the core and PDMS on the outer shell. This simplistic, single-step method implies an alternative for the generation of hybrid PDMS nanostructured material on a large scale with short processing time. 


\section{CHAPTER 7}

\section{SUMMARY AND FUTURE WORK}

\subsection{Summary}

Polydimethylsiloxane (PDMS) have been widely used in a variety of academic and industrial applications, because of their unique physical and chemical properties. The currently employed fabrication process were investigated and reviewed along with the identification of the advantages and the limitations of these techniques. Choosing the effective technique plays an important role in a relatively new area of research such as PDMS microdevices. Due to the increase in demand for designing and prototyping various PDMS structures and devices, new fabrication methods are needed to substitute lithography based methods initially adapted from IC fabrication to complement the demands of prototyping. The femtosecond laser microfabrication technique is identified to be most capable of achieving desired function of versatility, high quality and least number processing steps, along with being capable of producing true 3D features.

This thesis shows that the femtosecond laser rapid curing is a simple, fast and inexpensive technique for rapid prototyping of PDMS microstructures. Unlike other lithography processes, it does not require any mask, additives and it's configurable to design requirements; thereby it is suitable for rapid prototyping.

This thesis has reviewed typical PDMS prototyping process for the fabrication of micro and nano features and has suggested alternate novel method using laser technology for improving the quality and rate of production efforts. With respect to rate of PDMS device fabrication, the two main time -lining steps in the typical prototyping process are 
mold fabrication, curing. This thesis has introduced single step, maskless patterning of PDMS in micron-second timescale using femtosecond laser irradiation. Nanofiber -filled PDMS microstructures are also fabricated using the proposed method. It is evident from the Nanoindentation analysis that the elastic modulus of the reinforced structure has improved significantly, with the incorporation of nanofibers using the described method, which has potential of direct fabrication of reinforced PDMS micro/nano structures.

Hybrid PDMS 3D fibrous nanostructures are also generated using the proposed method. The versatility of this technique opens a new pathway to generate hybrid 3D fibrous nanostructures on any materials, which is limited in the current process. This simplistic, single-step method provides an alternative for the generation of hybrid nanostructured material on a large scale with short processing time. Since this technique of formation is at ambient condition, it is an efficient, simple and controllable process.

The proof - of- concept experiments followed, and femtosecond laser processing now appears to be a viable method for fabrication of pure PDMS and reinforced PDMS micro and nanostructures. Through study and experimentation, this thesis has specifically contributed to improving the rate of PDMS devices fabrication, particularly suitable for rapid prototyping. All the objectives outlined for this research are successfully achieved. The main contribution of this work are summarised below:

$\checkmark$ Established a maskless, single-step, non clean room manufacturing platform for the PDMS micro and nano structures/devices.

$\checkmark$ A new method to reinforce PDMS with nanofibers and construction of reinforced PDMS micro structures using femtosecond laser irradiation. 
$\checkmark$ Novel direct dry technique to generate hybrid 3D fibrous nanostructure on any material in large scale with short processing time.

\subsection{Future Work}

This research has achieved promising results for fabrication of pure PDMS, reinforced PDMS microstructures and hybrid PDMS 3D fibrous nanostructure generation. PDMS was selected for this thesis because of its widespread use in micro and nanotechnology applications. In the future, there is no reason this method could not be applied to any thermosetting resins such as acrylates and urethanes.

During this research study, piezoscanner was used to scan the laser beam in $\mathrm{XY}$

plane. A motor driven stage can be used to focus the laser in $\mathrm{Z}$ direction to create $3 \mathrm{D}$ features such as microwells, microvalves, nozzles, pumps in a single-step.

Finally, 3D hybrid fibrous nanostructures generation can be extended to various kinds of other metals/semiconductors. 


\section{REFERENCES}

1. P.Darion et al. "Microsystems in biomedical applications", J. Micromech. Microeng. 10. 2 (2000) 235-244.

2. G.A.Diaz-Quijada et al. "Surface modification of thermoplastics--towards the plastic biochip for high throughput screening devices", Lab on a Chip,7. 7(2007) 856-862.

3. C.W.Tsao et al. "Bonding of thermoplastic polymer microfluidics" Microfluid Nanofluid, 1.6 (2009) 1-16.

4. S.D.Burnside et al."Synthesis and properties of new poly (dimethylsiloxane) nanocomposites" Chem. Mater, 9.7 (1995) 1597-1600.

5. Q.Zhang et al.’In-situ synthesis of poly (dimethylsiloxane)-gold nanoparticles composite films and its application in microfluidic systems" Lab on a Chip, 8. 2 (2008) 352-357.

6. A.Govindaraju et al." Reinforcement of PDMS masters using SU-8 truss structures", $J$. Micromech. Microeng, 15 (2005) 1303-1309.

7. B.H.Jo et al." Three-dimensional micro-channel fabrication in polydimethylsiloxane (PDMS) elastomer” J. Microelectromechanical Systems, 9.1(2000) 76-81.

8. Y.Xia et al." Soft Lithography" Angewaldte Chemie International edition, 37.5(1998) $550-575$.

9. J.L.Wilbur et al. "Microfabrication by Microcontact Printing of Self-Assembled Monolayers", Adv. Mater, 6.78 (1994) 600-604.

10. A.Kumar et al." Patterned Self-Assembled Monolayers and Meso-Scale Phenomena", Acc. Chem. Res, 28.5 (1995) 219-226. 
11. J. L.Wilbur et al." Microcontact printing of self-assembled monolayers: applications in microfabrication", Nanotechnology, 7. (1996) 452- 457.

12. Y.Xia et al. "Pattern transfer: Self-assembled monolayers as ultrathin resists", Microelectronic Engineering, 32 (1996) 255-268.

13. H.A. Biebuyck et al. "Lithography beyond light: micro-contact printing with monolayer resists," J. Res. Develop, 41 (1997) 159-170.

14. A.Colas,"Silicones: preparation, properties and performance", 2005. http://www.dowcorning.com/content/publishedlit/01-3077.pdf.

15. M.J. Owens,’Why Silicones Behave Funny”, 2005. www.dowcorning.com

16. M.P.Wagner,’Reinforcing silicas and silicates” Rubber Chem. Technol. 49.3 (1976) 70374.

17. A.Colas et al." Silicone Biomaterials: History \& Chemistry \& Medical applications of silicones", 2005. www.dowcorning.com

18. A.Moshak et al."Silicone polymers in controlled drug delivery Systems - A review", Iranian Polymer Journal, 18. 4(2009) 279-295.

19. Polymer Data Handbook, Mark J., Oxford Univ. Press, NewYork (1999).

20. D.Armani et al. "Re-configurable Fluid Circuits by PDMS Elastomer Micromachining", “Twelfth Micro Electro Mechanical Systems (MEMS), Jan 21 (1999) 222 - 227.

21. http://www.dowcorning.com/applications/search/products/details.aspx?prod=04028007\& type $=$ prod.

22. Knovel Critical Tables (2nd Edition). Knovel/ Chemistry \& Chemical Engineering, (2008). 
23. A.Banerjee et al." Temperature distribution in different materials due to short pulse laser irradiation", Heat transfer engineering, 8. 26 (2005) 41-49.

24. P.-A.Auroux et al."Micro total analysis systems.2. Analytical standard operations and applications, 74 (2002) 2637-2652.

25. S.A. Ruiz et al. "Microcontact Printing: A tool to pattern" Soft Matter 3 (2007) 1-11.

26. A.Perl et al."Microcontact printing: limitations and achievements", Adv. Materials. 21.22 (2009) 2257-2268.

27. M. Nakano et al."Holographic laser scanners using generalized zone plates", Appl. Opt. 18 (1979) 3073-3074.

28. D. A. Kiewit. "Microtool fabrication by etch pit replication,"Rev. Sci. Instrum. 44. 12 (1973) 1741-1742.

29. B.D.Terris et al." Nanoscale replication for scanning probe data storage", Appl. Phys. Lett. 69 (1996) 4262-4264.

30. J.T.Fourkas et al. A review. "Multiphoton polymerization ", Materials today, 10. 6 (2007) 30-37.

31. H.Wu et al." Fabrication of complex 3D microchannel systems in PDMS", J.Amer. Chem. Soc. 125 (2003) 554-559.

32. J.M.K.Ng et al. "Components for integrated poly (dimethylsiloxane) microfluidic systems", Electrophoresis, 23. 20 (2002) 3461-3473.

33. J.R.Anderson et al. "Fabrication of topologically complex three-dimensional microfluidic systems in PDMS by rapid prototyping", Anal Chem, 72.14 (2000) 3158-3164. 
34. M.T.Raimondi, et al." Two-photon laser polymerization: from fundamentals to biomedical application in tissue engineering and regenerative medicine", J Appl Biomater Function Mater, 10. 1 (2012) 56-66.

35. S.D.Gittard et al. "Two-photon polymerization microstructuring in regenerative medicine", Front Biosci, 5 (2013) 602-609.

36. A.Doraiswamy et al. "Fabrication of microneedles using two photon polymerization for transdermal delivery of nanomaterials", J Nanosci Nanotechnol, 10 .10 (2010) 63056312.

37. A.Koroleva et al. "Fabrication of fibrin scaffolds with controlled microscale architecture by a two-photon polymerization-micromolding technique”, Biofabrication, 4.1 (2012).

38. C.A.Coenjarts et al.'Two-Photon Three-Dimensional Microfabrication of Poly (Dimethylsiloxane) Elastomer”, American Chemical Society, 16 (2004) 5556-5558.

39. J.C.McDonald et al. "Fabrication of microfluidic systems in poly (dimethylsiloxane)", Electrophoresis, 21.1 (2000) 27-40.

40. D.C.Duffy et al. "Rapid prototyping of microfluidic systems in poly (dimethylsiloxane)", Analytical Chemistry, 70. 23 (1998) 4974-4984.

41. H.Zheng et al."Picosecond Laser Ablation of Polydimethylsiloxane (PDMS)", International Manufacturing Science and Engineering Conference (ASME), Indiana, USA, October 4-7(2009).

42. V.M. Graubner, et al "Incubation and ablation behavior of poly (dimethylsiloxane) for 266nm irradiation”, Applied Surface Science, 198 (2002) 786- 790. 
43. H.Kim et al. "Reaction Sites on Polydimethylsiloxane Elastomer Surfaces in the Presence of Imidazole/Microwave Plasma; A Spectroscopic Study", Langmuir, 12 (1996).

44. Y.V.White et al. "Single-pulse ultrafast-laser machining of high aspect nano-holes at the surface of $\mathrm{SiO}_{2}$ ", Optics Express, 16.19 (2008) 14411-14420.

45. J.N.Lee et al.’Solvent compatibility of PDMS based microfluidic devices", Analytical chemistry, 75.23 (, 2003) 6544-6554.

46. Q.Zhang et al.’In-situ synthesis of poly (dimethylsiloxane)-gold nanoparticles composite films and its application in microfluidic systems", Lab on a Chip, 8. 2 (2008) 352-357.

47. B.Wang et al. "Chitosan-mediated synthesis of gold nanoparticles on patterned poly (dimethylsiloxane) surfaces", Biomacromolecules, 4 (2006) 1203-1209.

48. J.H. Kim et al."Olefin induced dissolution of silver salts physically dispersed in inert polymers and their application to olefin/paraffin separation“", J of membrane science, 241. 2(2004).

49. S.S.Ozdemir et al. "Catalytic polymeric membranes: Preparation and application", Applied Catalysis - a General, 307.2 (2006).

50. M.T.Sulak et al. "Amperometric glucose biosensor based on gold-deposited polyvinylferrocene film on Pt electrode", Biosensors and Bioelectronics, 21. 9(2006) $1719-1726$.

51. S. Dire et al."Sol-Gel Synthesis of Siloxane Oxide Hybrid Coatings $\left(\mathrm{Si}\left(\mathrm{CH}_{3}\right)_{2} \mathrm{O}-\mathrm{MOx}\right.$ : $\mathrm{M}=\mathrm{Si}, \mathrm{Ti}, \mathrm{Zr}, \mathrm{Al}$ ) with Luminescent Properties”, J of Materials Chemistry, 2 .239 (1992). 
52. H. Chen et al."'Direct Laser Writing of Microtunnels and Reservoirs on Nanocomposite Materials", Technical Proceedings of the NSTI Nanotechnology Conference and Trade Show, (2007).

53. J. N.Coleman et al.”Mechanical Reinforcement of Polymers Using Carbon Nanotubes", Advanced Materials, (2006) 689-706.

54. Z.J.Jheng et al.” Reinforcement of a poly (dimethylsiloxane) mold with high aspect ratio microstructures via a gas-liquid phase sol-gel process", J Micromech Microeng, 19. 4 (2009).

55. M.Mortan.” Rubber Technology" $4^{\text {th }}$ Edition/VanNostrand, Newyork, (1987).

56. H.J.Yang et al." Fabrication and application of high quality poly (dimethylsiloxane) stamps by gamma ray irradiation", J. Mater. Chem. 21(2011) 4279-4285.

57. D.J.T.Hill et al."Molecular weight changes and scission and crosslinking in PDMS on gamma radiolysis", Radiation physics and chemistry, 62 (2001).

58. A.O.Neill et al."Rapid curing of PDMS for microfluidic applications", Lab on a chip: Chips and Tips,(2006).

59. Y.S.Shin et al, "PDMS based micro PCR Chip with parlene coating", $J$ of Micromechanics and microengineering, 13.5 (2003).

60. S.W.Lee et al. "Shrinkage ratio of PDMS and its alignment method for the wafer level process" Microsystems Technologies,(2007).

61. A.Kumar et al, "Patterning self assembled monolayers, Applications in material science" Languтuir, 10. 5 (1994). 
62. A.Rosenthal et al. "Cell Patterning chip for controlling the stem cell microenvironment" Biomaterials, 28.21 (2007) 3208-3216.

63. S.W.Lee et al."Shrinkage ratio of PDMS and its alignment method for the wafer level process, Microsystem Technologies. (2007).

64. L.Kim et al. "Microfluidic arrays for logarithmically perfused embryonic stem cell culture", Lab on a Chip,6. 3 (2006) 394-406.

65. G.Vozzi et al.’Microfabricated PLGA scaffolds: a comparative study for application to tissue engineering", Materials Science and Engineering C, 20 (2002) 43 - 47.

66. . K.G.Klemic et al.”An air-molding technique for fabricating PDMS planar patch-clamp electrodes", Pflugers Arch, 449. 6 (2006) 564-72.

67. S.W.Lee et al."'Shrinkage ratio of PDMS and its alignment method for the wafer level process", Microsystem Technologies, 2007.

68. Jo et al.’Three-dimensional micro-channel fabrication in polydimethylsiloxane (PDMS) elastomer, J. Micromech. Syst, 9, 76-81 (1999).

69. H.B.Liu et al.’Templateless prototyping of polydimethylsiloxane microfluidic structures using a pulsed $\mathrm{CO}_{2}$ laser", J. Micromech. Microeng, 19 (2009).

70. R.Huang et al."Non-linear mechanical behavior of the elastomer polydimethylsiloxane (PDMS) used in the manufacture of microfluidic devices", (2005).

71. J.Zhang et al."Effect of exposure dose on the replication fidelity and profile of very high aspect ratio microchannels in SU-8", Lab on a Chip, 4. (2004) 646-653.

72. K. Venkatakrishnan et al." Femtosecond Pulsed Laser Direct Writing System”, Opt. Eng,41 (2002) 1441-1445, 2002. 
73. B.Tan et al."Synthesis of fibrous nanoparticle aggregates by femtosecond laser ablation in air", Optics Express, 17. 2 (2009) 1064 - 1069.

74. M Sivakumar et al. "Study of metallic fibrous nanoparticle aggregate produced using femtosecond laser radiation under ambient conditions", Nanotechnology, 21. 22 (2010).

75. P.A.George et al.'Integrated microfluidic devices for terahertz spectroscopy of biomolecules", Opt. Express, 16 (2008) 1577-1582.

76. C.B.Schaffer et al."Micromachining bulk glass by use of femtosecond laser pulses with nanojoule energy", Appl. Phys, A 76 (2003) 351-354.

77. M. Sakakura et al.'Heating and rapid cooling of bulk glass after photoexcitation by a focused femtosecond laser pulse", Optics Express, 15. 25(2007)16800-16807.

78. R.R.Gattass et al. "Micromachining of bulk glass with bursts of femtosecond laser pulses at variable repetition rates", Opt. Express, 14 (2006) 5279-5284.

79. S.Eaton et al."Heat accumulation effects in femtosecond laser-written waveguides with variable repetition rate", Opt. Express, 13 (2005) 4708-4716.

80. K.G.Yager et al."Temperature modeling of laser-irradiated azo-polymer thin films",J. Chem. Phys, 120.2 (2004) $1089-1096$.

81. Mark J E (ed) Polymer Data Handbook, New York / Oxford University Press, (1999).

82. J. Brewer et al." Pulsed laser desorption of alkali atoms from PDMS thin films", Applied Surface Science, 228. 1(2004) $40-47$.

83. K. Kruczala et al."Measuring Diffusion Coefficients of Nitroxide Radicals in Heterophasic Propylene-Ethylene Copolymers by Electron Spin Resonance Imaging”, Macromolecule, 44 .2 (2011) 325-333. 
84. T. Howard Thomas et al."Thermal analysis of polydimethylsiloxane. I. Thermal degradation in controlled atmospheres" ,Journal of Polymer Science Part A-2: Polymer Physics, 7.3 (2003) 537-549.

85. K. Chenoweth et al."Simulations on the thermal decomposition of a poly (dimethylsiloxane) polymer using the reactive force field", J. Am. Chem. Soc, 127.19 (2005) 7192-7202.

86. M.Liu et al.'Influences of heating temperature on mechanical properties of polydimethylsiloxane”, Sensors and Actuators A: Physical, 151. 1 (2009) 42 - 45.

87. R.S.Kane et al.'Patterning proteins and cells using soft lithography", Biomaterials, 20.23-24 (1999) 2363-2376.

88. A.Khademhosseini et al."Microscale technologies for tissue engineering and biology", Proc Natl Acad Sci U S A,103. 8 (2006) 2480-2487.

89. H.Nagai et al.’'Thermal conductivity measurement of liquid materials by a hot-disk method in short-duration microgravity environments", Mater Sci Eng A, 276. 1-2 (2000) $117-123$.

90. Zhang et al."Heat accumulation during high repetition rate ultrafast laser interaction: waveguide writing in borosilicate glass", J Phys Conf Ser, 59 (2007) 682-686.

91. D.A.Belfore, Laser Focus World 1.126 (1993).

92. L.Bokobza,'Elastomeric composites.I.Silicone composites”, J of Applied Polym Science, 93.5 (2004) $2095-2104$.

93. H.L.Cong et al." Photopatternable conductive PDMS materials for microfabrication", Ad Funct Mater, 18.13 (2008) 1912-1921. 
94. J.Engel et al. "Multi-walled carbon nanotubefilled conductive elastomers: materials and application to microtransducers", Conf Proc IEEE Micro Electro Mech Sy,(2006) 246249.

95. C.X.Liu et al."An embedded PDMS nanocomposite strain sensor toward biomedical applications", Conf Proc IEEE Eng Med Biol Soc, (2009) 6391-6394.

96. C.Luo et al.'Fabrication and application of silicon-reinforced PDMS masters, “J.Microelectron, 37. 10(2006) 1036-1046.

97. X.Z.Niu et al."Characterizing and patterning of PDMS-based conducting composites" , Adv.Mater, 19. 18 (2007).

98. Liu C.’Nanocomposite conductive elastomer: microfabrication processes and applications in soft-matter MEMS sensors”, Mater Res Soc Proc, (2007) 72-77.

99. S.D.Burnside et al."Synthesis and properties of new poly (dimethylsiloxane) nanocomposites, Chem Mater,9 (1995) 1597-1600.

100. B.Wang et al."Chitosan-mediated synthesis of gold nanoparticles on patterned poly (dimethylsiloxane) surfaces", Biomacromolecules, 4(2006) 1203-1209.

101. L.Bokobza.’The reinforcement of elastomeric networks by fillers“, Macromol Mater Eng, 289.7(2004) 607-621.

102. J.E.Mark."Ceramic-reinforced polymers and polymer modified ceramics", Polym Eng Sci, 36.24(1996) 2905-2920. 
103. C.H.Hu et al. "Resistance-pressure sensitivity and a mechanism study of multiwall carbon nanotubes networks/poly (dimethylsiloxane) composites", Appl Phys Lett, $93.3(2008)$.

104. M.A.Osman et al.'Reinforcement of poly (dimethylsiloxane) networks by montmorillonite platelets”, J Appl Polym Sci, 83.10(2002) 2175-2183.

105. M.A.Osman et al.’Reinforcement of poly (dimethylsiloxane) networks by mica flakes", Polymer, 42.15(2001) 6545-6556.

106. N.R.Raravikar et al.’Embedded carbon-nanotube-stiffened polymer surfaces", Small, $1.3(2005)$ 317-320.

107. J.E.Mark et al.’Simultaneous curing and filling of elastomers", Macromolecules, 17.12 (1984) 2613-2616.

108. S.K.Liao et al.'Phase behavior and mechanical properties of siloxane-urethane copolymer", J Polym Res, 12 .2(2005) 103-112.

109. T.B. Stachowiak et al. "Fabrication of porus polymer monoliths covalently attached to the walls of channels in plastic microdevices", Electrophoresis, 24.21 (2003).

110. R.T.kelly et al. "Phase changing sacrificial materials for solvent bonding of high performance polymeric capillary electrophoresis microchip", Anal chem, 77.11(2005).

111. P. Mao et al."Fabrication and characterization of $20 \mathrm{~nm}$ planar nanofluidic channels by glass-glass and glass-silicon bonding”, Lab on a Chip,5(2005) 837-844. 
112. W. Li et al. "Sacrificial polymers for nanofluidic channels in biological applications", Nanotechnology, 14(2003) 578-583.

113. Bilitewski et al. "Biochemical analysis with microfluidic systems", Analytical and Bioanalytical Chemistry, 377 (2003) 556-569.

114. H.A.Reed et al. "Fabrication of microchannels using polycarbonates as sacrificial materials", J. Micromech. Microeng, 11 (2001) 733-737.

115. S.Senadheera et al."Critical Time to Nucleation: Graphite and Silicon Nanoparticle Generation by Laser Ablation", Journal of Nanotechnology, (2009).

116. S.Jariwala et al."Single step self-enclosed fluidic channels via two photon absorption (TPA) polymerization”, Optics Express, 18.2(2010) 1630-1636.

117. A.Alubaidy et al.'Femtosecond laser material processing of electrically conductive reinforced polymer, Journal of Nanostructured Polymers and nanocomposites", $J$ of nanostructured polymers and nanocomposites,6.4 (2010) 123- 128.

118. N. Pinna et al."A chemical synthesis of yttria based crystalline and lamellar nanostructures and their formation mechanism", Small, 1.112(2005).

119. Z.Xu et al.'Rare earth fluorides nanowires/nanorods derived from hydroxides: hydrothermal synthesis and luminescence properties",Cryst. growth des,9.11 (2009) 4752-4758.

120. N. Zhang et al.'Lanthanide hydroxide nanorods and their thermal decomposition to lanthanide oxide nanorods", Materials Chemistry and Physics, 114.1(2009)160-167. 
121. K.Landfester. "Miniemulsion Polymerization and the Structure of Polymer and Hybrid Nanoparticles" Angew. Chem. Int. Ed. 48(2009) 4488 - 4507.

122. A.M.Schwartzberg et al. "Novel optical properties and emerging applications of metal nanostructures", J. Phys. Chem. C, 112.28(2008) 10323-10337.

123. M.Chen et al."Synthesis and self-assembly of fcc phase FePt nanorods", J. Am. Chem. Soc, 129.20(2007).

124. X.Duan et al."Indium phosphide nanowires as building blocks for nanoscale electronic and optoelectronic devices", Nature, (2001) 409: 66-69.

125. B.P.Vinayan et al. "Synthesis of graphene-multiwalled carbon nanotubes hybrid nanostructure by strengthened electrostatic interaction and its lithium ion battery application", J. Mater. Chem, 22( 2012) 9949-9956.

126. Sebastian Mackowski.”Hybrid nanostructures for efficient light harvesting”, J. Phys. Condens. Matter ,22.19(2010).

127. Shaoli Zhu et al. "Fabrication, and Applications of Hybrid Nanostructured Array", Journal of Nanomaterials. (2012).

128. A.I.Kuznetsov et al."Nanostructuring of thin gold films by femtosecond lasers" , Appl Phys A, 94(2009)221-230.

129. G.Miyaji et al."Origin of periodicity in nanostructuring on thin film surfaces ablated with femtosecond laser pulses", Opt Express, 16(2008) 16265-16271.

130. D.S.Ivanov et al.'The mechanism of nanobump formation in femtosecond pulse laser nanostructuring of thin metal films", Appl Phys A,92 (2008). 
131. R.P.de Campos et al."Raman imaging spectroscopic characterization of modified poly (dimethylsiloxane) for micro total analysis systems applications",Spectrochimica Acta Part A: Molecular and Biomolecular Spectroscopy, 100(2013) 67-71. 


\title{
A NEW APPROACH TO FABRICATE PDMS STRUCTURES USING FEMTOSECOND LASER
}

\author{
By \\ Hamsapriya Selvaraj \\ Bachelor of Engineering (Mechanical) \\ Bharathiyar University, India 2002 \\ Master of Engineering (Industrial) \\ Anna University, India 2005
}

\author{
A dissertation \\ presented to Ryerson University \\ in partial fulfillment of the requirement for the degree of \\ Doctor of Philosophy \\ in the program of \\ Aerospace Engineering
}

Toronto, Ontario, Canada, 2013

(C) Hamsapriya Selvaraj 2013 


\section{AUTHOR'S DECLARATION}

I hereby declare that I am the sole author of this dissertation. This is a true copy of the dissertation, including any required final revisions, as accepted by my examiners.

I authorize Ryerson University to lend this dissertation to other institutions or individuals for the purpose of scholarly research.

I further authorize Ryerson University to reproduce this dissertation by photocopying or by other means, in total or in part, at the request of other institutions or individuals for the purpose of scholarly research.

I understand that my dissertation may be made electronically available to the public.

\section{Hamsapriya Selvaraj}

Department of Aerospace Engineering

\section{Ryerson University}




\title{
ABSTRACT \\ A NEW APPROACH TO FABRICATE PDMS STRUCTURES USING FEMTOSECOND LASER
}

\author{
Doctor of Philosophy, 2013 \\ Hamsapriya Selvaraj \\ Department of Aerospace Engineering, Ryerson University
}

Polydimethylsiloxane (PDMS) is commonly used to prototype micro and nano featured components due to its beneficial properties. PDMS based devices have been used for diverse applications such as cell culturing, cell sorting and sensors. Motivated by such diverse applications possible through pure PDMS and reinforced PDMS, numerous efforts have been directed towards developing novel fabrication techniques. Prototyping 2D and 3D pure and reinforced PDMS microdevices normally require a long curing time and must go through multiple steps. This research explores the possibility of fabricating microscale and nanoscale structures directly from PDMS resin using femtosecond laser processing. This study offers an alternative fabrication route that potentially lead to a new way for prototyping of pure and reinforced PDMS devices, and the generation of hybrid nanomaterials.

In depth investigation of femtosecond laser irradiation of PDMS resin reveals that the process is highly intensity-dependent. At low to intermediate intensity regime, femtosecond laser beam is able to rapidly cure the resin and create micron-sized structures directly from PDMS resin. At higher intensity regime, a total break-down of the resin material occurs and leads to the formation of PDMS nanoparticles.

This work demonstrates a new way of rapid curing of PDMS resin on a microsecond timescale using femtosecond laser irradiation. The proposed technique permits maskless singlestep curing and is capable of fabricating 2D and 3D structures in micro-scale. Reinforced PDMS 
microstructures also have been fabricated through this method. The proposed technique permits both reinforcement and rapid curing and is ideal for fabricating reinforced structures in microscale. The strength of the nanofiber reinforced PDMS microstructures has been investigated by means of Nanoindentation test. The results showed significant improvement in strength of the material.

Hybrid PDMS-Si and hybrid PDMS-Al nanoparticle aggregate were generated using femtosecond laser. The results indicate that the hybrid PDMS nanostructures are clusters of nanoparticles that agglomerate and interweave three-dimensionally and also the possibility of formation of Si/Al nanoparticles enclosed in PDMS Shells. Presence of PDMS in the final hybrid structure is confirmed by micro-raman analysis. The versatility of our technique opens a new pathway to generate hybrid 3D fibrous nanostructures on any materials. 


\section{ACKNOWLEDGEMENT}

I wish to express my sincere appreciation to those who have contributed to this thesis and supported me in one way or the other during this amazing journey.

Foremost, I would like to express my sincere gratitude to my advisor Dr.Bo Tan for the continuous support of my Ph.D study and research, for her patience, motivation, enthusiasm, and immense knowledge. Her guidance helped me in all the time of research and writing of this thesis. I could not have imagined having a better advisor and mentor for my Ph.D study. Her advice on both research as well as on my career have been invaluable.

My sincere gratitude is reserved for Dr.Krishnan Venkatakrishnan for giving me the opportunity to carry out my doctoral research and for the financial support. I will never forget his support and for providing me numerous opportunities to learn and develop.

I would like to thank my examination committee: Dr.Muthukumaran Packirisamy (Concordia University), Dr.Huu Doan, Dr.Ziad Saghir, Dr.Puren Ouyang and Dr. Jeff Xi for their encouragement, insightful comments, and hard questions.

A special thanks to my family. Words cannot express how grateful I am to my parents and brother for all of the sacrifices that you've made on my behalf. Your prayer for me was what sustained me thus far.

I would like to acknowledge the most important person in my life - my husband Thirumurugan. Thank you for supporting me for everything, and especially I can't thank you enough for encouraging me throughout this experience. Thiru has been a constant source of strength and inspiration. There were times during the past five years when everything seemed hopeless and I didn't have any hope. I can honestly say that it was only his determination and constant encouragement that ultimately made it possible for me to see this project through to the 
end. Thiru has been a true and great supporter and has unconditionally loved me during my good and bad times. He has been non-judgmental of me and instrumental in instilling confidence. He has faith in me and my intellect even when I felt like digging hole and crawling into one because I didn't have faith in myself. These past several years have not been an easy ride, both academically and personally. I truly thank Thiru for sticking by my side, even when I was irritable and depressed. I feel that what we both learned a lot about life and strengthened our commitment and determination to each other and to live life to the fullest.

Special thanks to my beloved daughter Krishika and son Vihaan, who had to sacrifice their time and needs to support me in finishing my studies.

I would especially like to thank my brothers Senthilkumar, Jaganathan and my friend Murugesan for their unconditional support.

Last but not least I would like to give ultimate thanks to god. He guided and encouraged me. I thank him for his love and continuous support. Every breath I take and every moment I have are because of him. I would like to express the deepest thanksgiving to god and I pray he will continue to teach me and use me for his glory. Thank you, Lord. 


\section{LIST OF PUBLICATIONS}

$\checkmark$ Hamsapriya Selvaraj, Bo Tan and Krishnan Venkatakrishnan (2012) Self-Enclosed Nanofiber-Reinforced PDMS Channels Using Ultrafast Laser Irradiation, J. Micromechanical systems IEEE/ASME, Vol.21, Issue 5, pp: 1071 - 1076.

$\checkmark$ Hamsapriya Selvaraj1, Bo Tan and K Venkatakrishnan (2011) Maskless direct microstructuring of PDMS by femtosecond laser localized rapid curing, J. Micromech. Microeng, Vol.21, Issue 7.

$\checkmark$ Hamsapriya Selvaraj, Bo Tan and Kirshnan Venkatakrishnan(2011) Synthesis of nanofiber-filled polydimethylsiloxane using ultrafast laser irradiation, J Polym Res, Vol. 18, Issue 6, pp:1659-1665.

$\checkmark$ Hamsapriya Selvaraj, Bo Tan and Krishnan Venkatakrishnan (2012) Synthesis of hybrid PDMS-Si \& hybrid PDMS-Al fibrous nanostructured material using femtosecond laser ablation (Submitted to Polymer journal). 